\title{
DESIGNING A SIMULATOR FOR AN ELECTRICALLY-PUMPED ORGANIC LASER DIODE
}

\author{
A Thesis \\ presented to \\ the Faculty of California Polytechnic State University, \\ San Luis Obispo
}

\author{
In Partial Fulfillment \\ of the Requirements for the Degree \\ Master of Science in Electrial Engineering
}

by

Robert Hulbert

June 2019 
(c) 2019

Robert Hulbert

ALL RIGHTS RESERVED 


\section{COMMITTEE MEMBERSHIP}

TITLE: Designing a Simulator for an ElectricallyPumped Organic Laser Diode

AUTHOR: Robert Hulbert

DATE SUBMITTED: June 2019

COMMITTEE CHAIR: David Braun, Ph.D.

Professor of Electrical Engineering

COMMitTeE MEMBER: Xiaomin Jin, Ph.D.

Professor of Electrical Engineering

COMMITTEE MEMBER: Dennis Derickson, Ph.D.

Professor of Electrical Engineering 


\begin{abstract}
Designing a Simulator for an Electrically-Pumped Organic Laser Diode

Robert Hulbert
\end{abstract}

Organic semiconductors provide an alternative set of basis materials to fabricate electronic devices like PN Junctions, LEDs, and FETs. These materials have several benefits over traditional inorganic semiconductors including their mechanical flexibility, reliance on renewable resources, and inexpensive large-scale manufacturability. Despite the contemporary device implementations with organic semiconductors, a solid-state electrically-pumped organic laser diode does not exist. However, organically-based lasers do exist by utilizing the organic material strictly for optical gain. The challenge occurs when charge carriers appear in the organic material. The charge carriers must reach a concentration such that population inversion occurs producing optical gain. However, between the overlapping emission and absorption spectra of the organic material and insufficient carrier concentrations, positive optical gain remains elusive in electrically-pumped organic diodes. Organic device simulation provides a faster method of testing organic materials and device structures for positive optical gain based on known organic physics. The results generated from simulation provide key information in development of physical organic devices. This project produces a simulator capable of modeling current density and optical density with the intent of testing various device structures that allow for lazing in organic materials. 


\section{ACKNOWLEDGMENTS}

Thanks to:

- Bob and Sue Hulbert, for supporting me throughout my life, especially through the trials of my academic career.

- David Braun, for the constant intellectual support and motivation

- Juan Sanchez et. al, for generating and open sourcing DEVSIM

- Andrew Guenther, for uploading this template 


\section{TABLE OF CONTENTS}

Page

LIST OF TABLES ....................... . . . . ix

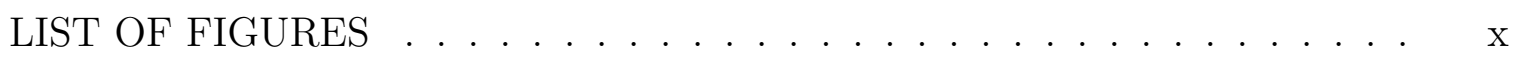

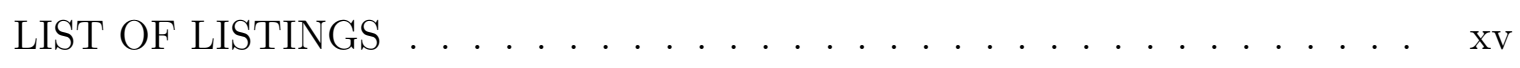
CHAPTER

1 Introduction . . . . . . . . . . . . . . . . . . . . 1

2 Available Software Platforms . . . . . . . . . . . . . . 5

$2.1 \quad$ Silvaco . . . . . . . . . . . . . . . . 6

2.2 COMSOL $\ldots \ldots \ldots \ldots \ldots \ldots \ldots \ldots \ldots \ldots \ldots \ldots \ldots \ldots \ldots$

2.3 DEVSIM . . . . . . . . . . . . . . . . . . . . . . . . . 7

2.3 .1 GMSH $\ldots \ldots \ldots \ldots \ldots \ldots \ldots$

$2.3 .2 \quad$ VISIT $\ldots \ldots \ldots \ldots \ldots \ldots \ldots$

3 Generating Necessary Models . . . . . . . . . . . . . . . . . . . . 11

3.1 Poisson . . . . . . . . . . . . . . . . . . . . . . . 11

3.1.1 Ohmic Boundary Condition _. . . . . . . . . . . 12

3.1.2 Schottky Boundary Condition . . . . . . . . . . 15

3.2 Carrier Models . . . . . . . . . . . . . . . . . . 16

3.2 .1 Thermal Equilibrium . . . . . . . . . . . . . . 16

3.2 .2 Current Continuity Equation $\ldots \ldots \ldots \ldots \ldots$

3.2 .3 Drift-Diffusion . . . . . . . . . . . . . . . . . 18

3.2 .4 Field-Dependent Mobility . . . . . . . . . . . . . 20

3.2 .5 Ohmic Boundary Condition _. . . . . . . . . . . 21

3.2.6 Schottky Boundary Conditions _. . . . . . . . . . 23 
3.3 Recombination . . . . . . . . . . . . . . . . . . . . 24

3.3.1 Spontaneous Emission . . . . . . . . . . . . . . . 24

3.3 .2 Net Stimulated Emission . . . . . . . . . . . . . . . . . 24

3.4 Optical Density . . . . . . . . . . . . . . . . . 26

3.4.1 Perfect Electric Conductor Boundary Condition . . . . . . 27

3.4 .2 Absorbing Boundary Condition _. . . . . . . . . 28

3.5 Photon Rate Equation . . . . . . . . . . . . . . . . . . . . . . . 29

3.5 .1 Emitted Power . . . . . . . . . . . . . . . 30

4 Developing in Python . . . . . . . . . . . . . . . . . . . . . 31

4.1 DEVSIM Solver . . . . . . . . . . . . . . . . . . . . 31

4.1 .1 Material Parameters And Units . . . . . . . . . . . . . . 32

4.1 .2 Python Model Format . . . . . . . . . . . . . . . . . 32

4.1 .3 Equation Building . . . . . . . . . . . . . . . . 34

4.1 .4 Contact Assembly . . . . . . . . . . . . . . . . . . 35

4.2 Helmholtz Solver . . . . . . . . . . . . . . . . . . 35

4.2 .1 Waveguide Refractive Index . . . . . . . . . . . . 35

$4.2 .2 \quad$ Optical Density . . . . . . . . . . . . . . . . . 38

4.3 Semiconductor Simulation . . . . . . . . . . . . . . . . . . 39

5 Results and Discussion . . . . . . . . . . . . . . . . . . . 41

5.1 Density Distributions . . . . . . . . . . . . . . . . . 44

5.2 I-V Curves . . . . . . . . . . . . . . . . . . . . . . . 49

5.3 P-V Curves . . . . . . . . . . . . . . . . 56

6 Lasing Simulation . . . . . . . . . . . . . . . . . . . . . . . . 61

6.1 Ohmic . . . . . . . . . . . . . . . 62

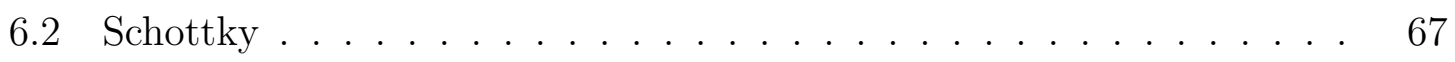


7 Conclusion and Future Work . . . . . . . . . . . . . . . . . . . 72

Bibliography ............................. 75

\section{APPENDICES}

A Useful Links . . . . . . . . . . . . . . . . . . 80

B Scharfetter-Gummel Derivation .............. 81

C Mode Propagation Constant .............. 83

D Lasing Plots . . . . . . . . . . . . . . . . 85 


\section{LIST OF TABLES}

Table

Page

5.1 Simulation Parameters . . . . . . . . . . . . . . . . . 42

5.2 Global Constants . . . . . . . . . . . . . . . 42

5.3 OC1C10-PPV Material Parameters . . . . . . . . . . 43

$5.4 \quad$ ITO Material Parameters . . . . . . . . . . . . . . . 43

5.5 Calcium Material Parameters ............... 43

6.1 Mobility Iterations . . . . . . . . . . . . . . . 61

6.2 Modified ITO Material Parameters . . . . . . . . . . . . . 62

6.3 Modified Calcium Material Parameters . . . . . . . . . . . 62

6.4 Mobility Mapping . . . . . . . . . . . . . . 63

7.1 Implemented Equations and Constituent Models . . . . . . . . . . 73 


\section{LIST OF FIGURES}

Figure

Page

1.1 Molecular Energy Levels and Band Equivalence . . . . . . . . . . . 2

1.2 Schematic of Polymer LED: Cathode (Electron Injector), Film (Emissive Layer), Anode (Hole Injector) . . . . . . . . . . . . . . . 3

2.1 Node Evaluation Components in 2D Mesh . . . . . . . . . . 8

2.2 Edge Evaluation Components in 2D Mesh . . . . . . . . . . 9

2.3 Element Edge Evaluation Components in 2D Mesh . . . . . . . . . 10

3.1 Ohmic Contact Energy Band Diagram for Intrinsic Semiconductor . 13

3.2 Ohmic Contact Energy Band Diagram for Doped Semiconductor . . 14

3.3 Schottky Contact Energy Band Diagram for Intrinsic Semiconductor 15

$4.1 \quad$ Full Execution Cycle of Simulator in 1D_PPV_diode.py . . . . . . . 39

5.1 LED Device Structure and Mesh Representation in Simulator . . . 41

5.2 Nodal Intrinsic Potential for Ohmic and Schottky Contacts . . . . . 44

5.3 Nodal Electron and Hole Densities for Ohmic Contacts . . . . . . . 45

5.4 Nodal Electron and Hole Densities for Schottky Contacts . . . . . . 46

5.5 Nodal Optical Densities for Ohmic and Schottky Contacts for Contact Refractive Indices of $1 \ldots$. . . . . . . . . . . . . . . 47

5.6 Nodal Optical Densities for Ohmic and Schottky Contacts for Calcium and ITO Refractive Indices . . . . . . . . . . . . . . . 48

5.7 I-V Plot of 1D Emissive Layer and Ohmic Contacts . . . . . . . . 49

$5.8 \quad$ Log I-V Plot of 1D Emissive Layer and Ohmic Contacts . . . . . . 50

5.9 I-V Plot of 1D Emissive Layer, Ohmic Contacts, and Field-Dependent Mobility .................... 51 
5.10 I-V Plot of 1D Emissive Layer, Ohmic Contacts, and Field-Dependent Mobility . . . . . . . . . . . . . . . 52

5.11 I-V Plot of 1D Emissive Layer and Schottky Contacts . . . . . . . . 52

5.12 I-V Plot of 1D Emissive Layer and Schottky Contacts . . . . . . . . 53

5.13 Simulated and Experimental I-V Plot of 1D Emissive Layer, Schottky Contacts, and Field-Dependent Mobility . . . . . . . . . . . . 54

5.14 Simulated and Experimental I-V Plot of 1D Emissive Layer, Schottky Contacts, and Field-Dependent Mobility . . . . . . . . . . . 55

5.15 P-V Plot of 1D Emissive Layer and Ohmic Contacts . . . . . . 56

5.16 Semi-Log P-V Plot of 1D Emissive Layer and Ohmic Contacts . . . 57

5.17 P-V Plot of 1D Emissive Layer and Ohmic Contacts and FieldDependent Mobility ............... 57

5.18 Semi-Log P-V Plot of 1D Emissive Layer and Ohmic Contacts . . . 58

5.19 P-V Plot of 1D Emissive Layer and Schottky Contacts . . . . . . 58

5.20 Semi-Log P-V Plot of 1D Emissive Layer and Schottky Contacts . . 59

5.21 Simulated and Experimental P-V Plot of 1D Emissive Layer and Schottky Contacts . . . . . . . . . . . . . . 59

5.22 Simulated and Experimental Semi-Log P-V Plot of 1D Emissive Layer and Schottky Contacts . . . . . . . . . . . . . . . . . 60

6.1 Laser Device Structure and Mesh Representation in Simulator . . . 61

6.2 G-V Plot of 1D Emissive Layer with Ohmic Contacts . . . . . . . 62

6.3 Electron Quasi Fermi Level at Low (Green), Mid (Yellow), and High (Orange) Mobilities at 25V . . . . . . . . . . . .

6.4 Hole Quasi Fermi Level at Low (Green), Mid (Yellow), and High (Orange) Mobilities at 25V . . . . . . . . . . . 64

6.5 Zoomed Electron Quasi Fermi Level at Low (Green), Mid (Yellow), and High (Orange) Mobilities at 25V . . . . . . . . . . . .

6.6 Zoomed Hole Quasi Fermi Level at Low (Green), Mid (Yellow), and High (Orange) Mobilities at 25V . . . . . . . . . . 66 
6.7 G-V Plot of 1D Emissive Layer with Schottky Contacts . . . . . . . 67

6.8 Electron Quasi Fermi Level at Low (Green), Mid (Yellow), and High (Orange) Mobilities at 25V . . . . . . . . . . . . 68

6.9 Hole Quasi Fermi Level at Low (Green), Mid (Yellow), and High (Orange) Mobilities at 25V . . . . . . . . . . . . . . 69

6.10 Zoomed Electron Quasi Fermi Level at Low (Green), Mid (Yellow), and High (Orange) Mobilities at 25V . . . . . . . . . . 70

6.11 Zoomed Hole Quasi Fermi Level at Low (Green), Mid (Yellow), and High (Orange) Mobilities at 25V . . . . . . . . . . . . . . 71

7.1 Exciton Rate Equation Including Singlet/Triplet Excitons and Charge Carrier Quenching [6] . . . . . . . . . . . . . . 74

7.2 Energy Balance Transport Model yielding Heat Transport [8] . . . . 74

C.1 Wave Solutions in Material . . . . . . . . . . . . . 83

C.2 Transcendental Equation from Matrix Determinant . . . . . . . . . 84

D.1 IV-Plot of 1D Emissive Layer (OC1C10) Ohmic Contacts Low Mobility 85

D.2 Log IV-Plot of 1D Emissive Layer (OC1C10) Ohmic Contacts Low Mobility .................... 85

D.3 PV-Plot of 1D Emissive Layer (OC1C10) Ohmic Contacts Low Mobility . . . . . . . . . . . . . . . 86

D.4 Log PV-Plot of 1D Emissive Layer (OC1C10) Ohmic Contacts Low Mobility ................... 86

D.5 GV-Plot of 1D Emissive Layer (OC1C10) Ohmic Contacts Low Mobility . . . . . . . . . . . . . . . . 87

D.6 IV-Plot of 1D Emissive Layer (OC1C10) Ohmic Contacts Mid Mobility 87

D.7 Log IV-Plot of 1D Emissive Layer (OC1C10) Ohmic Contacts Mid Mobility ....................... 88

D.8 PV-Plot of 1D Emissive Layer (OC1C10) Ohmic Contacts Mid Mobility 88

D.9 Log PV-Plot of 1D Emissive Layer (OC1C10) Ohmic Contacts Mid Mobility . . . . . . . . . . . . . . . . . . 89 
D.10 GV-Plot of 1D Emissive Layer (OC1C10) Ohmic Contacts Mid Mobility . . . . . . . . . . . . . . . . . . . . 89

D.11 IV-Plot of 1D Emissive Layer (OC1C10) Ohmic Contacts High Mobility 90

D.12 Log IV-Plot of 1D Emissive Layer (OC1C10) Ohmic Contacts High Mobility . . . . . . . . . . . . . . . . 90

D.13 PV-Plot of 1D Emissive Layer (OC1C10) Ohmic Contacts High Mobility . . . . . . . . . . . . . . . . . . . 9 91

D.14 Log PV-Plot of 1D Emissive Layer (OC1C10) Ohmic Contacts High Mobility . . . . . . . . . . . . . . . . . . 91

D.15 GV-Plot of 1D Emissive Layer (OC1C10) Ohmic Contacts High Mo-

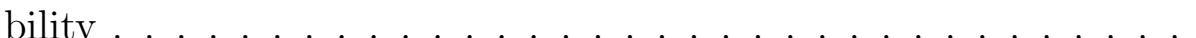

D.16 IV-Plot of 1D Emissive Layer (OC1C10) Schottky Contacts Low Mo-

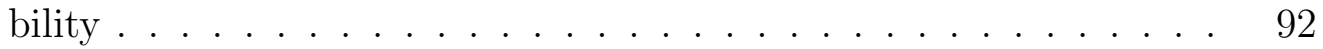

D.17 Log IV-Plot of 1D Emissive Layer (OC1C10) Schottky Contacts Low Mobility . . . . . . . . . . . . . . . . . . 93

D.18 PV-Plot of 1D Emissive Layer (OC1C10) Schottky Contacts Low Mobility . . . . . . . . . . . . . . . . . .

D.19 Log PV-Plot of 1D Emissive Layer (OC1C10) Schottky Contacts Low Mobility ........................

D.20 GV-Plot of 1D Emissive Layer (OC1C10) Schottky Contacts Low Mobility . . . . . . . . . . . . . . . . . .

D.21 IV-Plot of 1D Emissive Layer (OC1C10) Schottky Contacts Mid Mobility $\ldots \ldots \ldots \ldots$

D.22 Log IV-Plot of 1D Emissive Layer (OC1C10) Schottky Contacts Mid Mobility . . . . . . . . . . . . . . . . 95

D.23 PV-Plot of 1D Emissive Layer (OC1C10) Schottky Contacts Mid

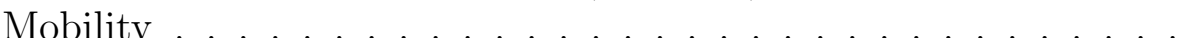

D.24 Log PV-Plot of 1D Emissive Layer (OC1C10) Schottky Contacts Mid Mobility . . . . . . . . . . . . . . . . 96

D.25 GV-Plot of 1D Emissive Layer (OC1C10) Schottky Contacts Mid Mobility . . . . . . . . . . . . . . . . . . . . . . 97 
D.26 IV-Plot of 1D Emissive Layer (OC1C10) Schottky Contacts High Mobility . . . . . . . . . . . . . . . . . . . 97

D.27 Log IV-Plot of 1D Emissive Layer (OC1C10) Schottky Contacts High Mobility . . . . . . . . . . . . . . . . . . 98

D.28 PV-Plot of 1D Emissive Layer (OC1C10) Schottky Contacts High Mobility . . . . . . . . . . . . . . . . . . . . 98

D.29 Log PV-Plot of 1D Emissive Layer (OC1C10) Schottky Contacts High Mobility . . . . . . . . . . . . . . . . . . . . . . 99

D.30 GV-Plot of 1D Emissive Layer (OC1C10) Schottky Contacts High Mobility . . . . . . . . . . . . . . . . . 99 


\section{LIST OF LISTINGS}

Listing Page

4.1 Model Format . . . . . . . . . . . . . . . . . . . . . . . . 32

4.2 Equation Builder . . . . . . . . . . . . . . . . . . 33

4.3 Transcendental Equation . . . . . . . . . . . . . . . . . 36

4.4 Matrix Construction . . . . . . . . . . . . . . . . . . . . 38

4.5 Solver Executions . . . . . . . . . . . . . . . . . . . . . 40 


\section{Chapter 1}

\section{INTRODUCTION}

Semiconducting polymers offer a wide range of mechanical properties from plasticity to elasticity, a larger configurable range of conductivity, and large, inexpensive production. These properties yield the potential for inexpensive, power efficient, bendable electronics. Televisions and smartphones with organic LED screens have already been introduced into the marketplace due to these properties. Furthermore, organically-based lasers, dye lasers, utilize polymers typically in solution to act as the lasing medium. These dye layers photo-pump the polymer solution which amplifies the power and shrinks the optical bandwidth of the optical input [1]. Despite these applications, an electrically-pumped organic laser diode remains undiscovered due to charge carrier quenching and statistical excitation phenomenon [1]. Physical synthesis attempts of this laser diode require significant time and resources. Instead, simulation offers a method to accelerate the rate of testing of various organic materials and device structures utilizing known physics. This report defends the following thesis statement: It is possible to construct a simulator capable of calculating carrier concentration, optical density, current-voltage characteristics, and luminous output for organic materials to test device structures for positive optical gain by electrical excitation.

Semiconducting organic materials conduct utilizing the long $\pi$-conjugated chains of monomer sub-structures. П-conjugated electrons form the alternating double or triple bonds in materials as their wave functions bend to overlap with each other. These bent wave functions provide the delocalization of electrons necessary to perform conduction perpendicular to the plane of the structure $[2,3]$. The electrons delocalize from 
the overlapping wave function, but the coherence distance does not extend as far depending on the length of the polymer. Similar to the band structure of inorganic semiconductors, polymer semiconductors utilize HOMO, highest occupied molecular orbital, and LUMO, lowest unoccupied molecular orbital, to refer to the carrier states in a short oligomer with discrete states. Once the polymers reach sufficient length , the discrete states become continuous yielding a band structure. In this case, LUMO and HOMO become synonymous with conduction and valence as shown in Fig. 1.1.

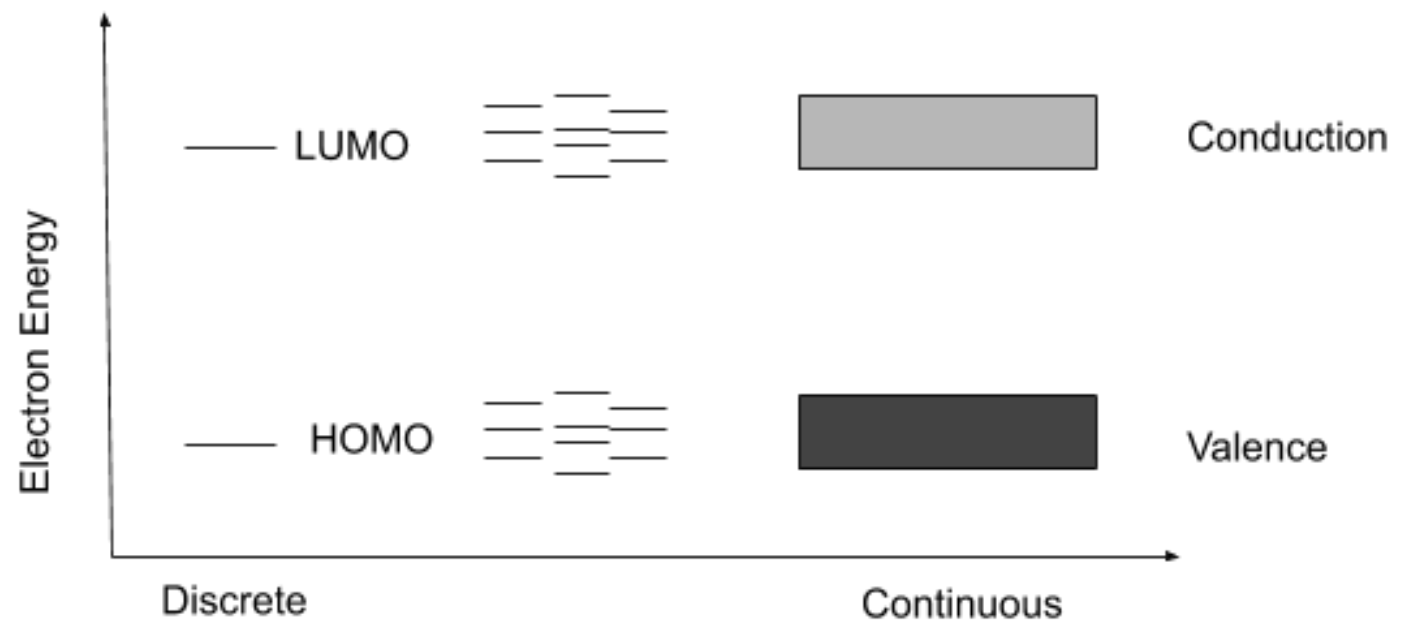

Figure 1.1: Molecular Energy Levels and Band Equivalence

An exciton occurs when an electron excites above the HOMO level and attracts a hole into a hydrogen-like object. The binding energy of this object is less than the energy gap between the HOMO and LUMO levels due to Coulombic attraction [2]. A singlet exciton with spin 0 decays into either a photon with energy corresponding to the binding energy or into the free hole-electron pair. A triplet exciton may also form with spin 1 that only has non-radiative decays to lower energy levels.

Besides excited states, polymer semiconductors also differ from traditional semiconductors in charge transport. Traditional inorganic semiconductors form rigid crystal lattices that remain more unaffected by Coulombic fields generated by charge carriers 
than polymer semiconductors. Due to the flexibility of the polymer chain, a higher coupling between the molecular structure and charge carriers forms as the structure bends to ensure the lowest energy. This shielding of the charge carrier forms a polaron, a charge carrier with higher mass and lower mobility than an electron or hole in an inorganic material [3].

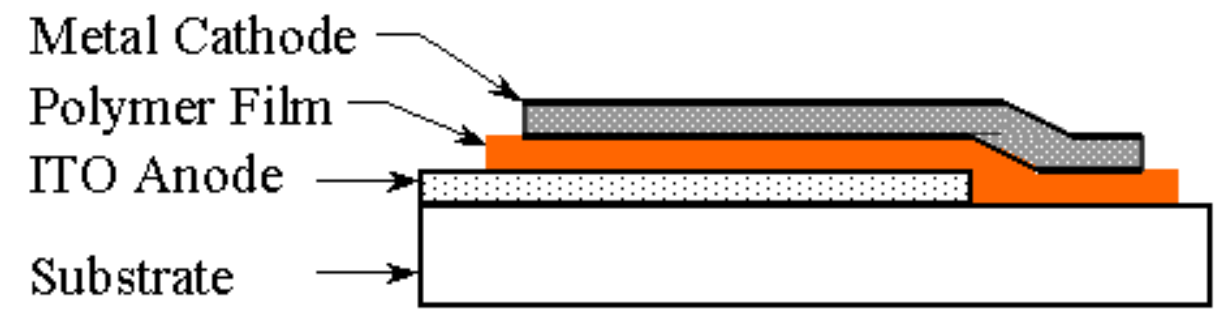

Figure 1.2: Schematic of Polymer LED: Cathode (Electron Injector), Film (Emissive Layer), Anode (Hole Injector)

Polarons and excitons form the mechanisms which allow an organic LED to electroluminesce, Fig. 1.2. The polarons carry the charges from the metal contacts to the emissive layer where excitons form based on statistical selection rules. Singlet excitons emit a photon with energy equivalent to their binding energy while triplet excitons decay via vibrational relaxing, phonon generation. The statistical selection rules dictate that a singlet to triplet generation follows a 1:3 rule when an exciton forms [1]. Furthermore, these triplet excitons absorb photons produced by singlet excitons and dissipate the energy non-radiatively [1]. For an electrically-pumped organic laser diode, the major challenge occurs in generating population inversion allowing positive optical gain despite the lower mobilities of polarons and selection rules producing singlet excitons. Simulation offers a faster development cycle than physical synthesis to test various device structures and organic materials with the goal of positive optical gain. 
The semiconductor industry utilizes simulation to design and test different devices and materials prior to physical implementation to save on development time and production cost. Simulators rely on known semiconductor physics and material parameters to reproduce results obtained empirically. Reference [5] details the simulation of edge-emitting lasers and VCSELs including the models, material parameters, and results. It further notes the efficiency limits and temperature effects in these devices. Despite the advances in inorganic laser diode simulation, organic laser diodes, due to their inherent difficulty, have minimal results. However, simulation work in OLEDs continues to increase for use in flexible screens. Organic material simulation includes exciton populations and field-dependent mobility [6]. Multiple commercial simulator solutions offer direct implementations or variants of these models for device simulation. However, his project decided to build an open-source simulator as it offered more flexibility and availability. 
Chapter 2

\section{AVAILABLE SOFTWARE PLATFORMS}

Due to finite storage and computational capabilities of modern computing, simulators discretize the physical domain where the device modeling occurs. Finite Element, Finite Volume, and Finite Difference constitute the three main types of device modeling techniques available. All three methods rely on a mesh that discretizes the spatial domain by points and lines. The Finite Element method solves the user-specified difference equations at the finite "element" whether it be a point, line, or a higher order discretization with a linear combination of basis functions of the equations. The Finite Volume method solves difference equations by assuming quantity conservation within a finite volume composed of the spatial features of the mesh. The simplest method, Finite Difference, directly solves any difference equation specified on the mesh. Finite Difference typically represents the easiest method to implement, but does not handle irregular meshes well. The Finite Element method allows for more flexibility of complex geometries as higher order terms can be added more easily in the solution summation, but requires more knowledge of the mathematical solution space to implement [7]. The Finite Volume method handles nonlinearities and transport equations well, but only solves equations that handle the flux of conserved quantities through a volume [7]. Silvaco and COMSOL represent commercial simulators that may use one or more of the methods above. This project uses the open source project, DEVSIM, due to availability and expandability, despite only implementing the Finite Volume method. 


\subsection{Silvaco}

Silvaco provides an extensive platform for 2D device simulation, ATLAS [8]. ATLAS contains multiple inter-connected sub-modules useful for developing a simulation of an electrically-pumped organic laser diode: Quantum, Luminous, Organic Display, and Laser [9]. The Quantum module utilizes a self-consistent Schrodinger-Poisson model to simulate quantum transport and confinement in semiconductor quantum wells. The Luminous package models photo-generation and absorption in devices utilizing geometric ray-tracing to track photon travel accounting for reflections, refractions, polarization, and dispersion. Organic Display simulates charge transport and recombination in organic materials utilizing the Frenkel-Poole hopping mechanism for transport and accounting for exciton-exciton interactions. Finally, the Laser module models spontaneous emission, stimulated emission, optical gain, strain in quantum wells, and optical density. These four modules provide the necessary bases to begin testing various structures/materials to generate an electrically-pumped organic laser diode. This project did not use Silvaco ATLAS as it was not monetarily available at the time of development.

\subsection{COMSOL}

COMSOL offers another platform to simulate an electrically-pumped organic laser diode. This platform contains several modules that would serve as a basis to begin structural/material testing of this diode: Electromagnetic, Semiconductor, and Wave Propagation [10]. The Electromagnetic module provides all of Maxwell's equations which would be useful for vacuum and conductive media simulation. The Semiconductor module offers the Poisson equation, Current Continuity equations, recombination, and doping. Finally, the Wave Propagation module offers models to simulate photon 
generation and flow through different media. Between the need for other models such as Frequency-Dependent Mobility and Schottky Barriers as well as the inaccessibility of the COMSOL models mentioned above due to limitations in the current contract, this project did not utilize COMSOL.

\subsection{DEVSIM}

DEVSIM offers an open-source Finite Volume solver written in $\mathrm{C}++$ to conduct physics simulations. The platform operates utilizing the control volume approach and evaluates any equation of the form in (2.1) [11]. This equation generates three types of expressions: Node, Edge, and Element. The Node model describes any expression that can be evaluated on a node in the mesh and integrates the expression over the node volume, Fig. 2.1. The Edge model describes any expression evaluated on the edge between two nodes on the mesh and integrates the expression as flux through a surface area, Fig. 2.2. The Element model describes any Edge model that depends on more information than the values at both nodes such as directional contributions from other edge models, Fig. 2.3. From this, DEVSIM built a series of APIs to build a mesh, add node, edge, or element models to construct a series of partial differential equations, solve the generated matrices from the equations, and output the results [11].

The $\mathrm{C}++$ APIs can be directly utilized, but have also been wrapped with python allowing for quicker development in a scripting language. DEVSIM provides several benefits including the ability to contribute to the source if needed, easily adding more complex models to the simulator, and cooperation with other open source projects that specialize in meshing and visualization in Sec. 2.3.1 and Sec. 2.3.2 [11]. DEVSIM

relies on another open source platform known as SYMDIFF which operates as a 
natural language parser for mathematical equations. This tool evaluates complex mathematical expressions and their derivatives, allowing for ease of incorporation of new models as they can be written in a string format and parsed by SYMDIFF as long as they contribute to (2.1). The user provides all the necessary physical models, Sec. 3, in string format for SYMDIFF. After SYMDIFF parses the models, the user generates the Control Volume compliant equations from the parsed models. The DEVSIM solver then executes on the built equations yielding the numerical solutions, Sec. 4.

$$
\int \frac{\partial X}{\partial t} d r+\int \vec{Y} \cdot d s+\int Z d r=0
$$

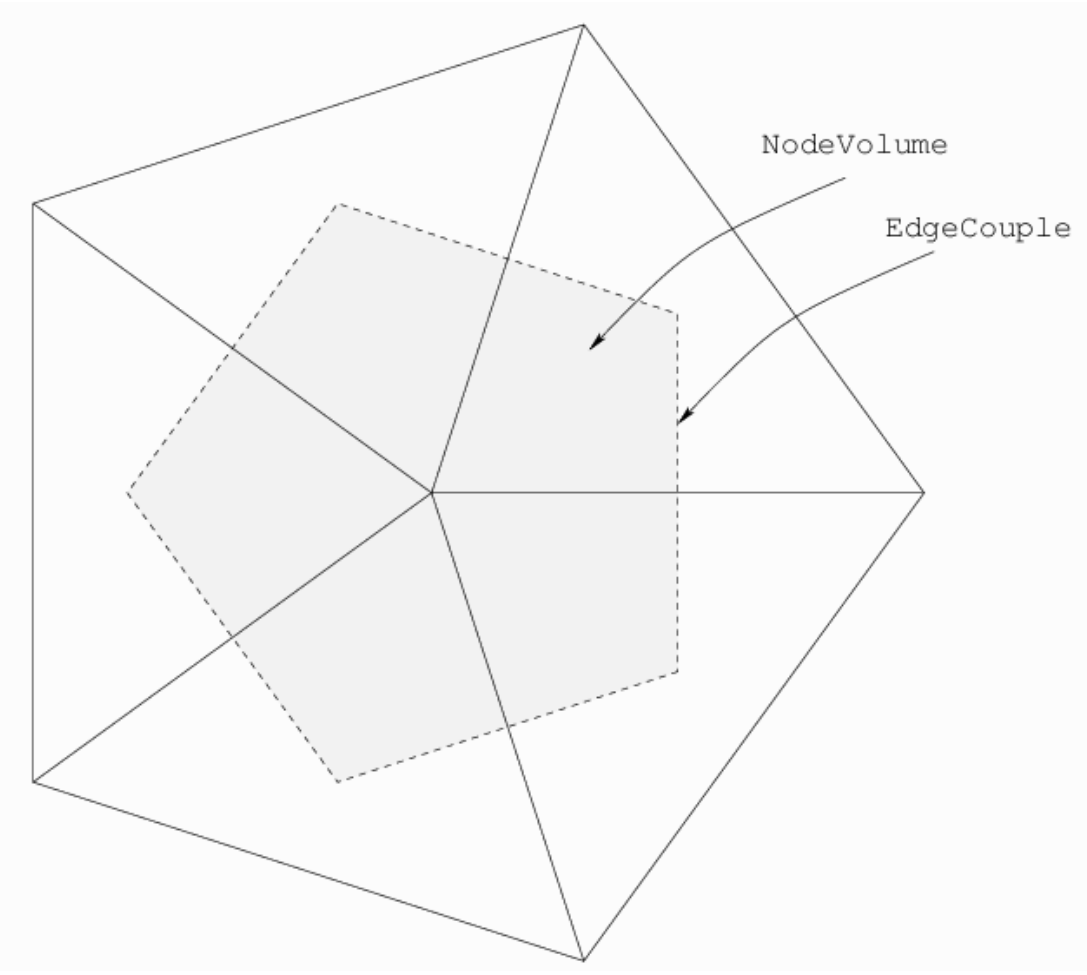

Figure 2.1: Node Evaluation Components in 2D Mesh [11] 


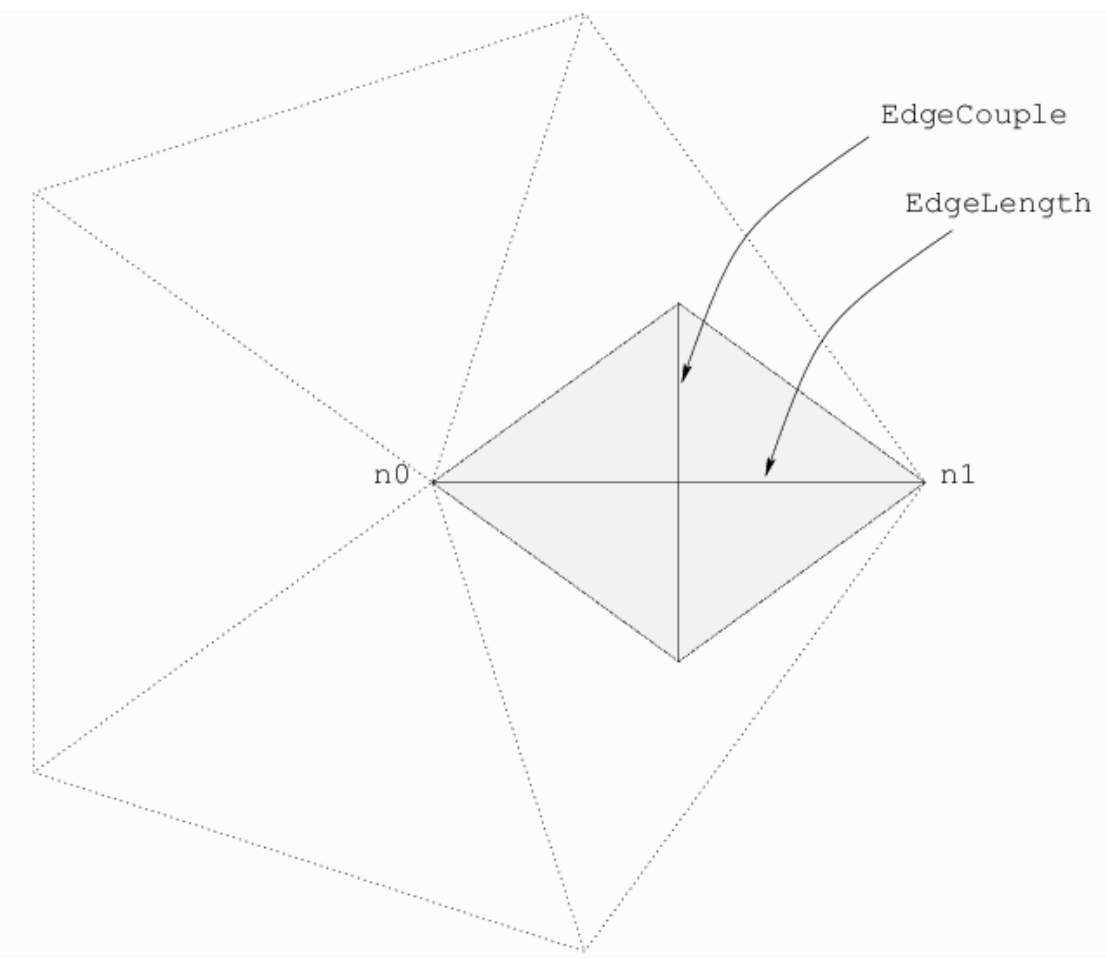

Figure 2.2: Edge Evaluation Components in 2D Mesh [11]

\subsubsection{GMSH}

The open-source meshing software, GMSH, provides a scripting platform to generate 1D, 2D, and 3D meshes. GMSH accepts a .geo file which contains the geometric information of the structure to be simulated and overlay a corresponding mesh utilized by DEVSIM to simulate the desired device. The geo file allows the user to specify points, curves (1D), surfaces (2D), and volumes (3D) to generate their structure. Furthermore, these geometric components can be grouped into a "Physical" aggregation DEVSIM may reference to specify the bulk region, the electrical contacts, and the interfaces between different materials. GMSH then overlays mesh nodes (0D), edges (1D), triangles (2D), and tetrahedrons (3D) to discretize the constructed geometry. 


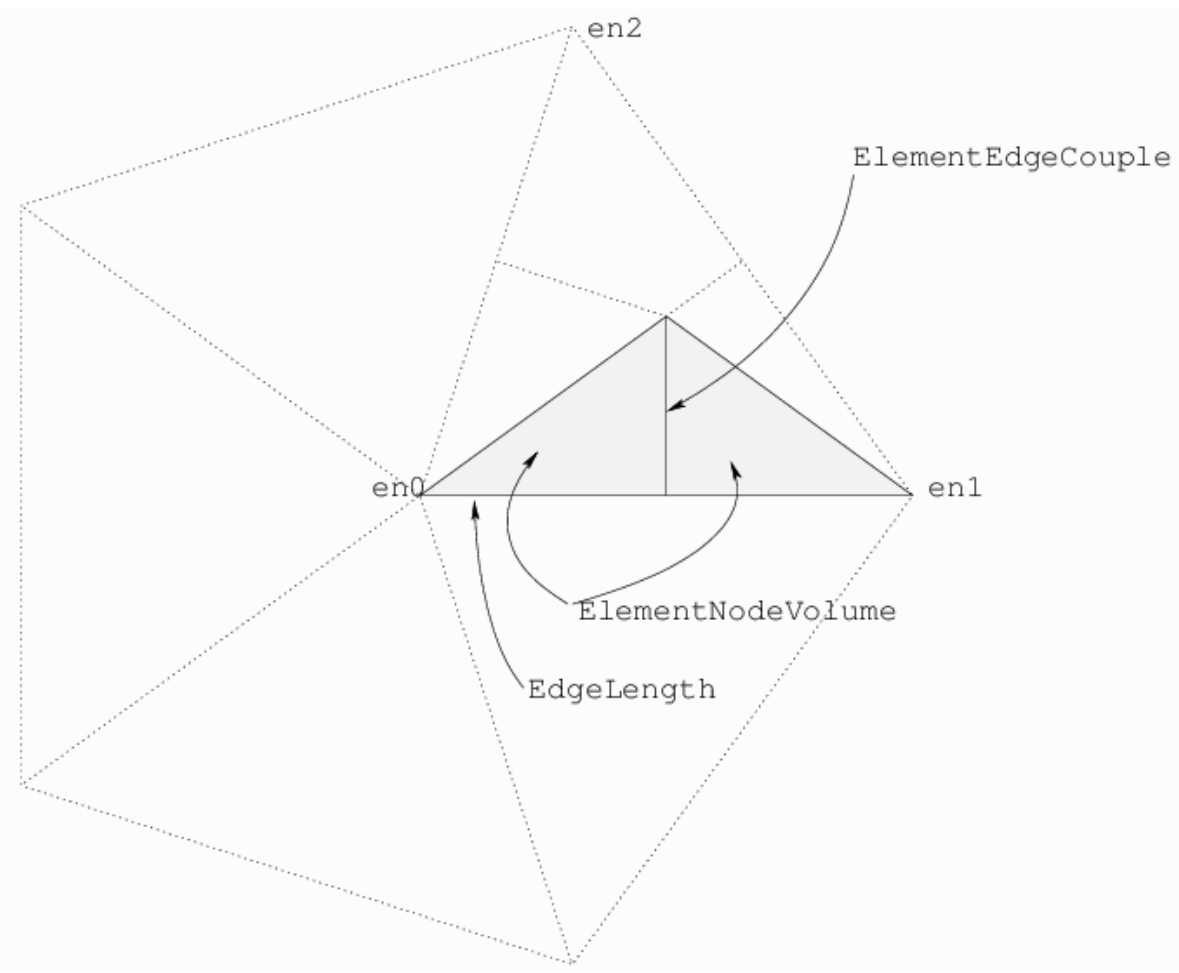

Figure 2.3: Element Edge Evaluation Components in 2D Mesh

[11]

\subsubsection{VISIT}

DEVSIM produces a file type, .vtk, as one of its several output formats. VISIT, produced by Lawrence Livermore National Laboratory, extracts from this file the models evaluated by DEVSIM [12]. These models include the intrinsic models produced by DEVSIM such as the node position, edge lengths, unit directions, etc. and the models generated by the user such as the charge at a node or the current through an edge. VISIT supports data analysis of the processed models including scalar operations, binning, and plotting of one up to three imported models at a given time. This flexibility of analysis shows charge concentration and optical density of the device structure during operation. 
Chapter 3

\section{GENERATING NECESSARY MODELS}

The mesh discretization (GMSH), solving method (DEVSIM), and visualization (VISIT) provide the necessary building blocks to begin building the physics to simulate the device. The user-defined differential equations describe the desired physics and information that the solver produces. The Poisson and Current Continuity equations describe the electrostatic potential, carrier concentrations, and carrier flux through the desired organic device. The recombination equations allow for the production of photons which, when included with the photon rate equation, describe generated, emitted, and absorbed electromagnetic energy in the device. The Helmholtz equation unlike the other equations relies on an external 1-D complex eigenvalue and transcendental equation solver as the DEVSIM solver does not provide these additional solvers. The Helmholtz equation represents the wave-like nature of photons and determines the density and confinement within the device.

\subsection{Poisson}

The Poisson Equation which relates potential to charge concentration starts with Gauss' Law. The creation of an Electric Field results from the presence of net charge within a given volume as shown in (3.1) [13]. When in matter, the permittivity of free space must be replaced by the permittivity of the desired material resulting in Electric Displacement (3.2). A scalar field (potential) produces an Electric Field in the absence of a Magnetic Field (3.3). The equation created from substituting (3.3) into (3.1) is known as Poisson's Equation (3.4) [13] [14]. Furthermore, the net charge 
density results from the difference between the electron density, hole density, and the net doping of acceptors and donors in the material (3.5). The particular solution of the Poisson equation arrives from the application of boundary conditions, Ohmic or Schottky, and self-consistent solving with the carrier models.

$$
\begin{gathered}
\nabla \cdot \vec{E}=\frac{\rho}{\epsilon_{0}} \\
\vec{D}=\epsilon * \vec{E} \\
\vec{E}=-\nabla \psi \\
\nabla^{2} \psi=-\frac{\rho}{\epsilon} \\
\rho=q *\left(p-n+N_{d}-N_{a}\right)
\end{gathered}
$$

\subsubsection{Ohmic Boundary Condition}

The Ohmic boundary condition physically represents a metal-semiconductor junction where the metal work-function and semiconductor conduction or valence band match exactly yielding a zero potential drop across the junction. This boundary condition applied to the Poisson equation specifies a Dirichlet boundary condition for the electrostatic potential $((3.16))$ setting the potential to the band edge at the contact of the device [14] [15]. For an intrinsic semiconductor, the conduction and valence band 
density of states dictate the Ohmic contact potential shown in (3.7) and (3.6) [14] - However, for a doped semiconductor, the donors and acceptors density of states directs the Ohmic contact potential resulting in (3.9) and (3.8) [14]. The intrinsic carrier concentration marks the zero energy reference band edge as shown in (3.10).

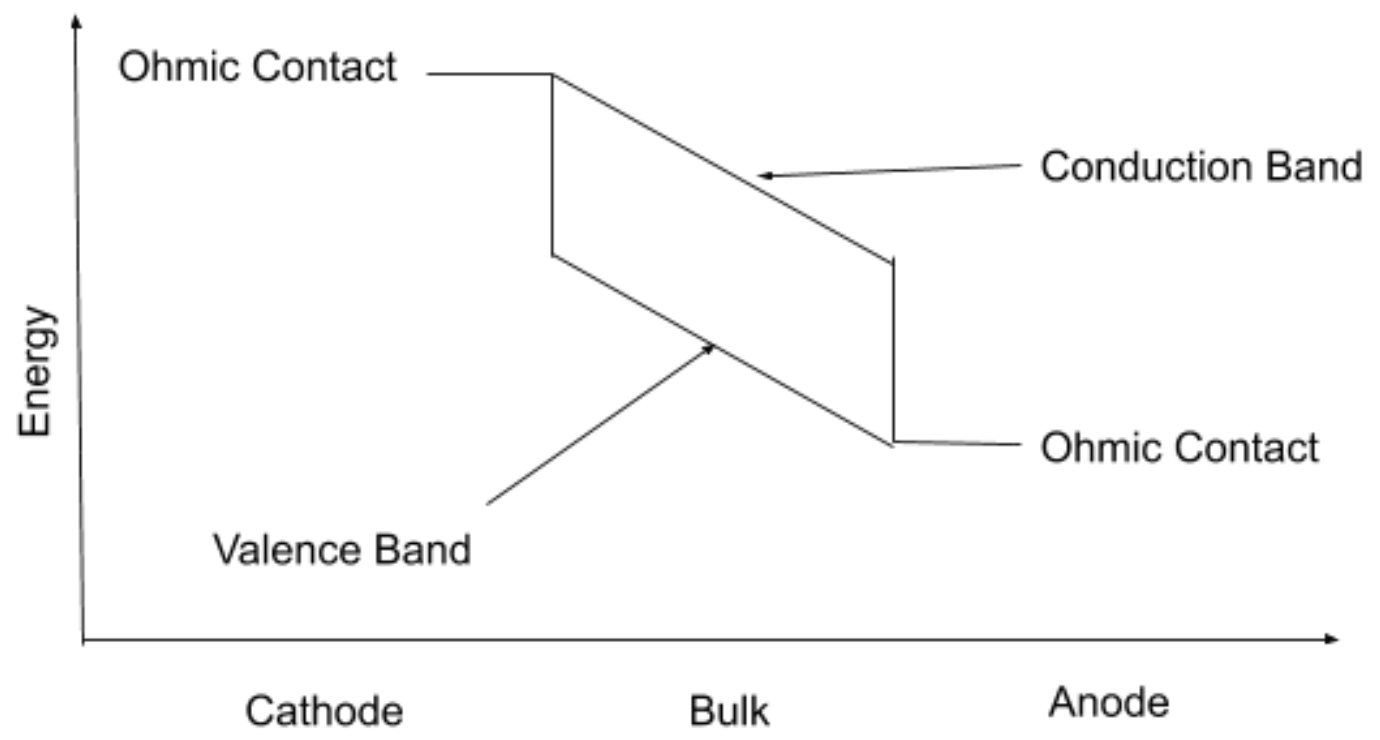

Figure 3.1: Ohmic Contact Energy Band Diagram for Intrinsic Semiconductor

$$
\begin{aligned}
& \psi=V_{a p p l}-\frac{k T}{q} \ln \left(\frac{N_{v}}{n_{i}}\right) \\
& \psi=V_{a p p l}+\frac{k T}{q} \ln \left(\frac{N_{c}}{n_{i}}\right)
\end{aligned}
$$

In Fig. 3.1, the Ohmic contacts attached to the conduction and valence band represent (3.7) and (3.6) with the device operating in forward bias. As characteristic of Ohmic contacts, the electrostatic potential at the contact matches the band edge. 


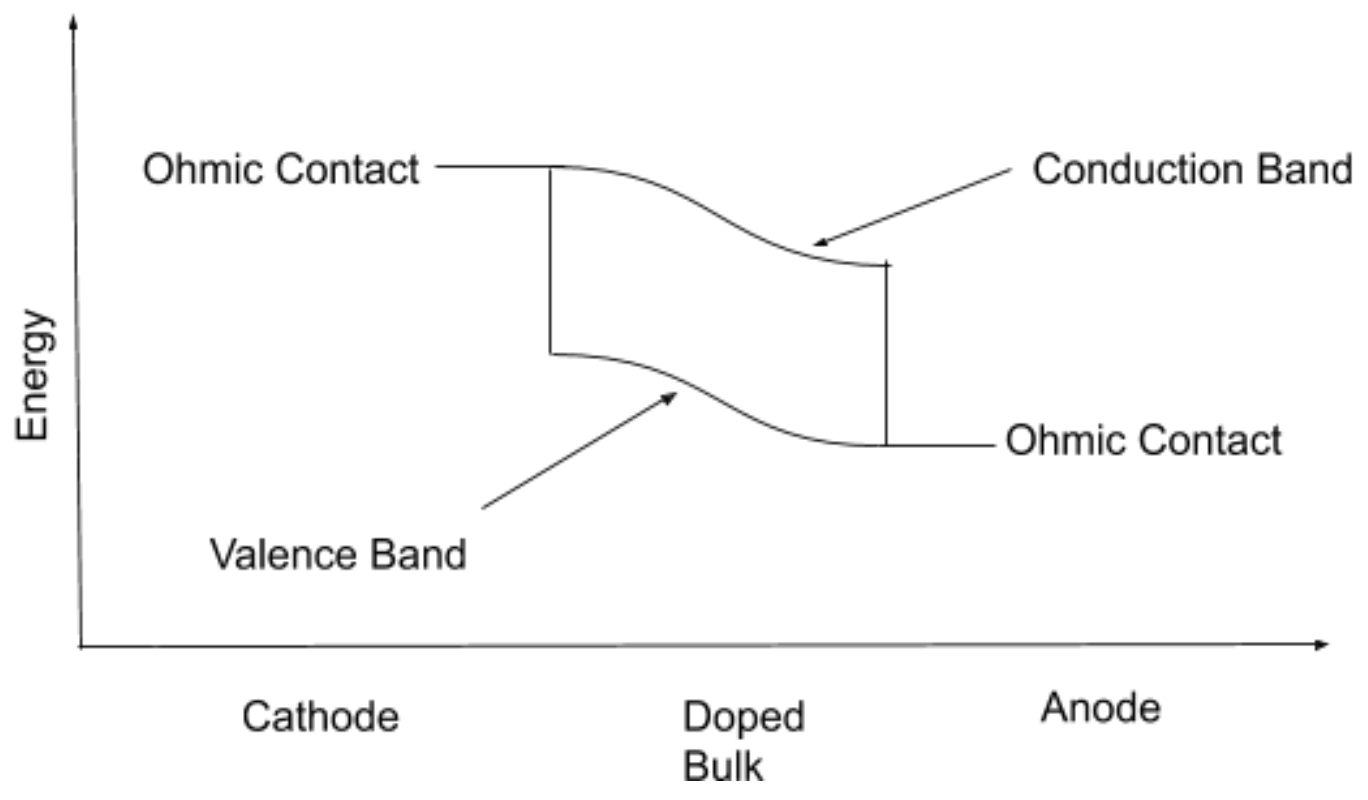

Figure 3.2: Ohmic Contact Energy Band Diagram for Doped Semiconductor

$$
\begin{gathered}
\psi=V_{a p p l}-\frac{k T}{q} \ln \left(\frac{N_{a}}{n_{i}}\right) \\
\psi=V_{a p p l}+\frac{k T}{q} \ln \left(\frac{N_{d}}{n_{i}}\right) \\
\psi=V_{a p p l}
\end{gathered}
$$

Similar to Fig. 3.1, Fig. 3.2 demonstrates the physical interpretations of (3.8) and (3.9). However, band bending occurs in a doped semiconductor due to the abrupt junction and change in carrier charge polarity. The Ohmic contacts continue to set the electrostatic potential to the band edge at the contact. 


\subsubsection{Schottky Boundary Condition}

The Schottky boundary condition provides a more realistic Dirichlet boundary condition for the Poisson equation. Typically, at the interface between a metal-semiconductor junction, a potential drop forms due to the unequal energies of the work-function and band-edge between the metal and semiconductor. The Schottky boundary condition accounts for this potential difference and corrects the Ohmic boundary condition with this difference between the energies as shown in the n-type (3.11) and p-type (3.12) Schottky boundaries [16].

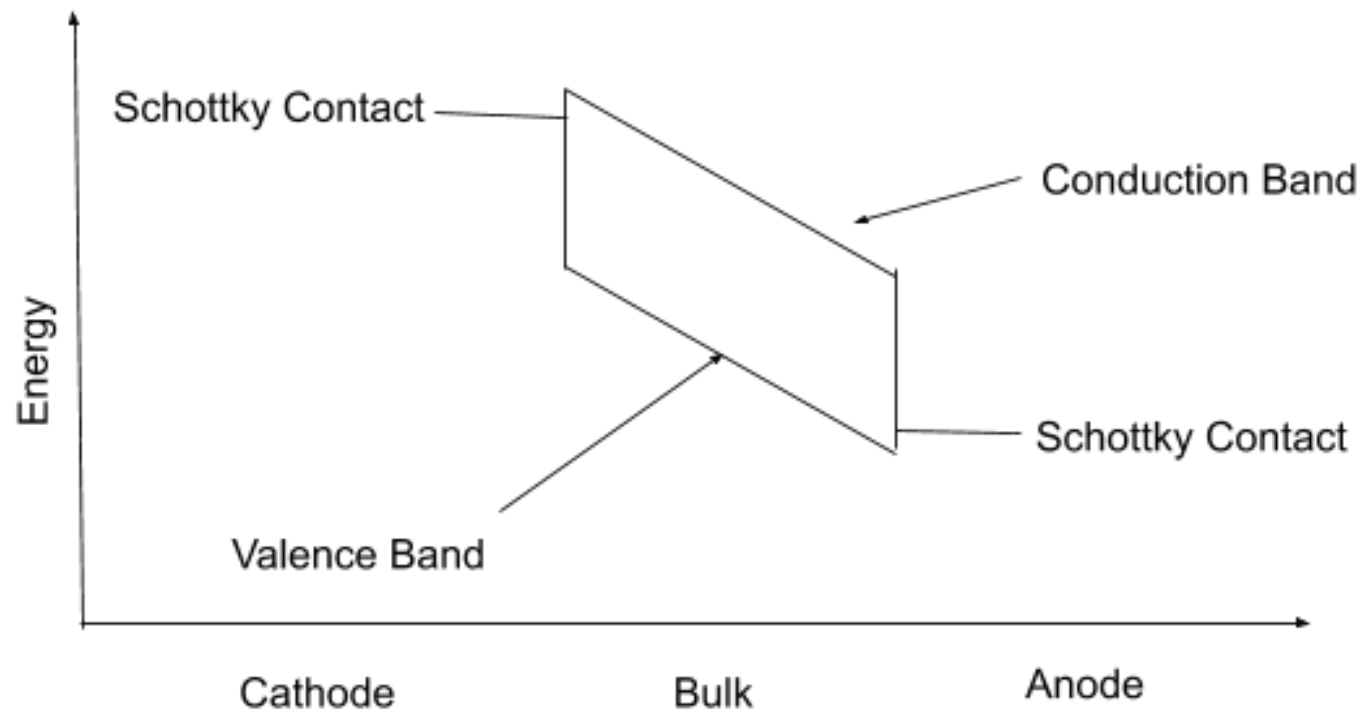

Figure 3.3: Schottky Contact Energy Band Diagram for Intrinsic Semiconductor

$$
\psi=V_{a p p l}+\frac{k T}{q} \ln \left(\frac{N_{c}}{n_{i}}\right)+\left(\psi_{m}-E_{c}\right)
$$




$$
\psi=V_{a p p l}+\frac{k T}{q} \ln \left(\frac{N_{v}}{n_{i}}\right)+\left(\psi_{m}-E_{v}\right)
$$

Fig. 3.3 demonstrates the difference between Schottky and Ohmic contacts with the potential difference between the bands and contact value. (3.11) and (3.12) account for this difference, in ${ }_{m}-E_{c, v}$.

\subsection{Carrier Models}

The electron and hole concentrations arise from two separate mechanisms: equilibrium and injection. The equilibrium concentrations arise from the electron and hole Fermi levels in thermal equilibrium. The injected carriers arise from the current flowing in through the contacts which may be dominated by the Drift-Diffusion current in the bulk material or contact current in the case of Schottky contacts.

\subsubsection{Thermal Equilibrium}

The electron and hole densities at equilibrium must equate as the thermal promotion of an electron to the conduction band yields a hole in the valence band [14]. A single (intrinsic) carrier results from the product of the electron and hole densities as the product remains constant with constant temperature (3.13). The electron (3.14) and hole densities (3.15) instead rely on the intrinsic carrier concentration and the electrostatic potential (3.16) within the material (3.17) (3.18) [14].

$$
n p=n_{i}^{2}=N_{c} N_{v} \exp \left(-\frac{E_{g}}{k T}\right)
$$




$$
\begin{gathered}
n=N_{c} * \exp \left(\frac{E_{c}-E_{f}}{k T}\right) \\
p=N_{v} * \exp \left(\frac{E_{f}-E_{v}}{k T}\right) \\
\psi=E_{i}-E_{f} \\
n=n_{i} * \exp \left(\frac{\psi}{k T}\right) \\
p=n_{i} * \exp \left(-\frac{\psi}{k T}\right)
\end{gathered}
$$

\subsubsection{Current Continuity Equation}

Under an applied bias, excess carriers from the contacts inject into the semiconductor and the total current through the device must be conserved given by the current continuity equation. Ampere's Law (with Maxwell's addition) (3.19) dictates that the Magnetic Field results from either the free current in a material or an oscillating Electric Displacement. The current continuity equation, however, derives from applying the divergence operator to Ampere's Law and the vector calculus identity that the divergence of a curl is zero (3.20). This equation when separated by the carriers produces the electron (3.21) and hole (3.22) current continuity equations as well as the relation between them, recombination [13]. 


$$
\begin{gathered}
\nabla \times \vec{H}=\vec{J}+\frac{\partial \vec{D}}{\partial t} \\
0=\nabla \cdot \overrightarrow{J_{n}}+\nabla \cdot \vec{J}_{p}+\frac{\partial \rho}{\partial t} \\
\nabla \cdot \vec{J}_{n}-\frac{\partial n}{\partial t}=q R \\
\nabla \cdot \vec{J}_{p}+\frac{\partial p}{\partial t}=-q R
\end{gathered}
$$

\subsubsection{Drift-Diffusion}

The current generated in the semiconductor bulk seen in the current continuity equations comes from two mechanisms: Drift and Diffusion. Under an external bias, charged carriers experience a force generating a current. This form of current generation known as Drift current follows Ohm's Law (3.23) [13]. As such, positively-charged holes flow in the direction of the bias and negatively-charged electrons flow against

the direction of the bias. Aside from Drift, current also forms from the concentration gradient of these carriers, diffusion. The Diffusion current follows from Fick's law (3.24) with diffusion coefficient D shown in (3.25). Together, the Drift-Diffusion model classically describes the current in a semiconductor for electrons (3.26) and holes (3.27) [13].

$$
\overrightarrow{J_{n, p}}=\sigma_{n, p} \vec{E}=q \mu_{n, p}(n, p) \vec{E}
$$




$$
\begin{gathered}
\overrightarrow{J_{n, p}}=(+,-) q D_{n, p} \nabla(n, p) \\
D_{n, p}=\frac{k T}{q} \mu_{n, p} \\
\overrightarrow{J_{n}}=q \mu_{n} n \vec{E}+q D_{n} \nabla(n) \\
\overrightarrow{J_{n}}=q \mu_{p} \vec{E}-q D_{p} \nabla(p)
\end{gathered}
$$

However, the Drift-Diffusion model must be discretized to satisfy the most accurate and least computationally complex differential operators on the grid [17]. The Scharfetter-Gummel discretization method produces the electron and hole currents shown in (3.28) and (3.29) by utilizing the Bernoulli Function, $B$, shown in (3.30) and voltage difference, $t$, shown in (3.31) [17]. The Scharfetter-Gummel method assumes that the current and electric field on an edge remain constant and then solves the Drift-Diffusion equation for the carrier concentration. After finding the analytical solution (the exponential in the Bernoulli function), the constant current and electric fields relate the two carrier concentrations at the nodes of the edge. Solving for the current between the two nodes yields the Scharfetter-Gummel discretized currents. For the full derivation of these discretized currents using the Scharfetter-Gummel method, see Appendix B [18].

$$
J_{n}=\frac{\mu_{n} k T}{x_{i+1}-x_{i}} *\left(n_{i+1} * B(t)-n_{i} * B(-t)\right)
$$




$$
\begin{gathered}
J_{p}=\frac{\mu_{p} k T}{x_{i+1}-x_{i}} *\left(p_{i+1} * B(-t)-p_{i} * B(t)\right) \\
B=\frac{x}{\exp (x)-1} \\
t=q \frac{\psi_{i+1}-\psi_{i}}{k T}
\end{gathered}
$$

\subsubsection{Field-Dependent Mobility}

The mobilities of electrons and holes in the Drift-Diffusion model for organic materials depend on the Electric field through the device. Due to the absence of a rigid lattice, organic semiconductors offer mechanical flexibility while maintaining conductance, however, this system also produces a higher coupling of carrier-phonon interactions. A new carrier known as a polaron with higher mass and lower mobility forms from the electrons and holes moving through the structure bending the molecular backbone as they move. Mathematically, this system produces lower, field-dependent electron and hole mobilities. While performing simulations and experiments of single and double carrier organic device structures, Blom demonstrated that these organic mobilities show a root dependence on the DC electric field through the material [19]. Depending on the disordering of the material, the mobilities fit closer to the Gaussian Disorder Model or the Correlated Disorder Model shown in (3.32) and (3.33) [19] [20]. The equation parameters $\sigma, C$, and $a$ represent the width of the Gaussian density of states, the site-spacing, and the intersite-spacing. 


$$
\begin{aligned}
& \mu_{c}=\mu_{\text {inf }} \exp \left(-\left(\frac{2 \sigma}{3 k T}\right)^{2}+C\left(\left(\frac{\sigma}{k T}\right)^{2}-2.25\right) \sqrt{E}\right) \\
& \mu_{c}=\mu_{\text {inf }} \exp \left(-\left(\frac{3 \sigma}{5 k T}\right)^{2}+.78\left(\left(\frac{\sigma}{k T}\right)^{2}-2\right) \sqrt{\frac{e a E}{\sigma}}\right)
\end{aligned}
$$

\subsubsection{Ohmic Boundary Condition}

As with the Poisson equation, the current continuity equations require boundary conditions to attain a specific solution. The Ohmic boundary condition, when applied to carrier injection, specifies an infinite contact recombination velocity and space charge neutrality [15], meaning charge density continuity on either side of the junction. However, the work-function of a metal does not change with an applied bias resulting in the carrier density remaining at its equilibrium concentrations at the contact. This system manifests a Dirichlet boundary condition for the electron and hole densities such that their concentrations must remain at the thermodynamic equilibrium densities at the contact as seen in (3.34) and (3.35) [15].

$$
\begin{aligned}
& n=n_{0} \\
& p=p_{0}
\end{aligned}
$$

The contact equilibrium concentrations depend on the doping of the material. The concentrations derive from the Mass Action law (3.36) and space-charge neutrality (3.37). In a doped semiconductor, these equations produce a polynomial equation 
with quadratic solutions that represent the concentrations shown in (3.38) and (3.39) [14]. The placing of the potential boundary condition matches the energy band equivalent of these concentrations.

$$
\begin{gathered}
n p=n_{i}^{2} \\
\rho=\left(p-n+N_{d}-N_{a}\right)=0 \\
n=\frac{N_{d}-N_{a}}{2}+\sqrt{\left(\frac{N_{d}-N_{a}}{2}\right)^{2}+n_{i}^{2}} ; p=\frac{n_{i}^{2}}{n} \\
p=\frac{N_{a}-N_{d}}{2}+\sqrt{\left(\frac{N_{a}-N_{d}}{2}\right)^{2}+n_{i}^{2}} ; n=\frac{n_{i}^{2}}{p}
\end{gathered}
$$

In an intrinsic semiconductor, the acceptor and donor ions represent minimal contribution yielding $n=p=n_{i}$. However, the placement of the potential on a specific band edge raises the carrier concentration associated with the band to the band density of states value as shown in (3.40) and (3.38) with the Mass Action Law yielding the complementary carrier concentration.

$$
\begin{aligned}
& n=N_{c} ; p=\frac{n_{i}^{2}}{n} \\
& p=N_{v} ; n=\frac{n_{i}^{2}}{p}
\end{aligned}
$$




\subsubsection{Schottky Boundary Conditions}

The Schottky boundary condition forgoes the assumption of infinite contact recombination velocity and requires the carrier concentrations at the semiconductor side of the contact to rely on the current density across the junction [15]. The carrier density on the metal side of the contact remains at the thermal equilibrium concentration, but a finite contact recombination velocity stipulates a current density across the junction. Thermionic emission and diffusion constitute the two current mechanisms that form this current density normal to the junction, $\overrightarrow{J_{n, p}} \cdot \hat{n}$. Thermionic emission accounts for the current generated by an applied voltage which raises or lowers the Fermi-level of the semiconductor causing carrier flow shown in (3.43) and (3.44). The thermionic recombination velocity, $v_{c}$, developed by Cromwell and Zhe accounts for the potential barrier effects on the flux through the junction [21] [20]. Diffusion accounts for the current generated by a carrier concentration gradient. By instituting the thermionic boundary condition after the diffusion equilibrium conditions (discussed further in Sec. 4.3), the thermionic and diffusion theories run in series including the contributions from both mechanisms [21].

$$
\begin{gathered}
v_{n, p}=\sqrt{\frac{k T}{2 \pi m_{n, p}}} \\
\vec{J}_{n} \cdot \hat{n}=-q v_{n}\left(n-n_{0}\right) \\
\vec{J}_{p} \cdot \hat{n}=q v_{p}\left(p-p_{0}\right)
\end{gathered}
$$




\subsection{Recombination}

After separating the current continuity equations by their carriers, the constant term that balances the two equations relies on recombination. Recombination accounts for all the possible interactions that occur between holes and electrons. This project

implements Spontaneous Emission and Stimulated Emission for photon production and gain modeling.

\subsubsection{Spontaneous Emission}

Electron-Hole pairs combine to produce excitons and excitons with spin 0, singlets, radiatively decay producing a photon equivalent to the binding energy. Generation and recombination of carrier pairs equate at thermal equilibrium 3.45, but with the application of a bias, excess carriers increase the recombination rate above equilibrium as shown in (3.46) [22] [6]. The constant, $B_{r}$, represents the bi-molecular radiative recombination rate.

$$
\begin{gathered}
R_{s p}=G=B_{r} n_{0} p_{0} \\
R_{s p}=B_{r} n p-G=B_{r}\left(n p-n_{0} p_{0}\right)
\end{gathered}
$$

\subsubsection{Net Stimulated Emission}

Stimulated emission occurs when a photon interacts with an exciton stimulating the emission of another photon. This interaction yields two photons with the same energy and phase. However, this process experiences competition from the reverse process, 
stimulated absorption, where a photon excites an Electron-Hole pair. (3.47) and (3.48) represent the stimulated emission and absorption rates [23]. The rates rely on $\beta$, the Einstein coefficients representing the probability of transition; the density of electrons and holes in the proper band for the transition; and the density of photons at the desired optical energy. Einstein showed $B_{21}$ and $B_{12}$ equate which leads to the reduction seen in (3.49). Optical gain in a laser occurs when the net rate between stimulated emission and absorption rates reach a positive value (3.49) [23]. $\rho_{c} \mathrm{dn}$ $\rho_{v}$ represent the density of states and $f_{e}\left(E_{e}\right)$ and $f_{p}\left(E_{p}\right)$ represent the carrier Fermi function of the carrier quasi-Fermi energy for the carriers in [23]. Population inversion marks the threshold of positive gain where more electrons exist in the excited state than the ground state, $f_{e}\left(E_{e}\right)-f_{p}\left(E_{p}\right)>0$. This gain changes with position through the material due to changes in carrier concentrations. The Stimulated Emission expression 3.51 relies on the modal gain which calculates the average gain over the bulk factoring the effects of the optical density produced by the Helmholtz as seen in 3.50.

$$
\begin{gathered}
r_{s t}=\beta_{21} n_{2} p_{1} \\
r_{a b}=\beta_{12} n_{1} p_{2} \\
g=r_{s t}-r_{a b}=\beta_{21} \rho_{c}\left(E_{e}-E_{c}\right) \rho_{v}\left(E_{v}-E_{p}\right)\left(f_{e}\left(E_{e}\right)-f_{p}\left(E_{p}\right)\right) \\
G_{m}=\frac{\int g\|E\|^{2}}{\int\|E\|^{2}}
\end{gathered}
$$




$$
R_{s t}=\frac{c}{n_{e} f f} G_{m} S
$$

\subsection{Optical Density}

Langevin recombination and Stimulated Emission produce photons which propagate as waves in the cavity. The Helmholtz equation describes the steady-state optical density within the cavity. Applying the curl operator to Ampere's Law (3.19) and incorporating Faraday's Law (3.52) produces the electromagnetic wave equation (3.53) when applying the curl of the curl identity. This equation describes the elec-

tromagnetic fields as perpetual propagating waves in a material. The steady-state Helmholtz equation derives from the Fourier transform of the electromagnetic wave equation (3.54). The frequency dependence of the Helmholtz equation requires a separate instantiation in the simulator for each desired optical wavelength and cavity mode.

$$
\begin{gathered}
\nabla \times \vec{E}=-\frac{\partial \vec{B}}{\partial t} \\
-\nabla^{2} \vec{E}+\frac{1}{c^{2}} \frac{\partial^{2} \vec{D}}{\partial^{2} t}=0 \\
\nabla^{2} E_{w}(x, y, z)+k^{2} \epsilon E_{w}(x, y, z)=0
\end{gathered}
$$

The Helmholtz equation describes the optical density, $E_{w}$, in the transverse plane and accounts for optical cavity effects, $k^{2} \epsilon$ or $k^{2}\left(n^{2}+n_{e f f}^{2}\right)$, that increases the optical gain 
of the device $[6,22,24,25]$. The complex eigenvalue solver retrieves the fundamental mode of the optical density from the Helmholtz equation after solving for the effective refractive index, $n_{\text {eff }}$. The effective refractive index describes the effects of multiple layers of varying refractive indices on the optical density. The multiple layers generate a series of nonlinear equations that produce a transcendental equation which provides the value of the effective refractive index discussed further in Sec. 4.2.1. The resulting optical density (fundamental mode) produces the modal factor which describes the cavity mode effect on that specific optical wavelength discussed further in Sec. 4.2.2. The stimulated recombination rate in Sec. 3.3.2 utilizes the modal factor with the optical gain to produce the modal gain for that wavelength. This project did not implement laser diode noise analysis nor modal dispersion, so these components of the polarity, $P$, in the electric displacement, $D$, are neglected from $[6,22,24,25]$. The noise analysis includes the Langevin noise produced by spontaneous emission, Sec. 3.3.1, and the modal dispersion includes the effects of impurities on traveling waves with different momentum vectors.

\subsubsection{Perfect Electric Conductor Boundary Condition}

The optical cavity of a laser physically requires two reflective boundaries that confine the coherent light and augment the optical gain. The perfect electric conductor boundary condition describes the reflective nature of the ends of the cavity. A solid perfect electrical conductor (PEC) with infinite conductivity has zero internal Electric field as the surface charge of the conductor negates the field. With the contact specified as a PEC and optical density conserved across the optical junction, the PEC boundary condition states that the optical density must be zero at the contact (3.55). This boundary condition does not account for optical density emission as it stipulates perfect reflection of the incident wave. Optical emission physically occurs 
due to the transmission properties of the materials as they do not perfectly reflect the optical wave. Sec. 3.5 discusses the emission of electromagnetic radiation due to transmission through the boundary.

$$
E_{w}=0
$$

\subsubsection{Absorbing Boundary Condition}

The absorbing boundary condition (ABC) does not designate a physical condition of the simulation. Optical waves approach zero intensity as their propagation distance reaches infinity. However, a computational domain can not simulate infinite space, so the $\mathrm{ABC}$ provides a method to truncate the computational domain and retrieve the optical information that occurs in that infinite distance. The ABC solves the Helmholtz equation (3.54) using evanescent instead of propagative wave solutions absorbing the optical power and minimizing reflections back into the computational domain $[26]$.

$$
\frac{\partial E_{w}}{\partial x}+i \frac{w}{k} \sqrt{1-\frac{c^{2} w^{2}}{k^{2}}} E_{w}=0
$$

However, the $\frac{c^{2} w^{2}}{k^{2}}$ term represents waves close to the normal of the surface at small values. The zero-order Taylor expansion of (3.56) approximates the appropriate boundary condition used to truncate the domain (3.57) and in one dimension represents the exact solution to the wave equation [26]. This boundary condition did not find use in the current project, but remains implemented in the external 1-D Helmholtz solver.

$$
\frac{\partial E_{w}}{\partial x}+i \frac{w}{k} E_{w}=0
$$




\subsection{Photon Rate Equation}

The Helmholtz equation yields the optical density distribution inside the optical cavity, but does not account for the power emanated from the device. The Electromagnetic Energy Conservation equation, (3.58), derives from Ampere's Law (3.19) with the application of the dot product of the Electric Field [27]. This equation de-

scribes the change in energy in a volume, $\frac{\partial U}{\partial t}$, as the power emanated from the surface containing the volume, $\nabla \cdot \vec{u}$, and the power generated within the volume $\vec{E} \cdot \vec{J}$.

However, this equation only describes classical electromagnetism. Reference [28] shows the full derivation of the photon rate equation shown in (3.62) which demonstrates a similar format to the electromagnetic energy conservation equation. The source term includes the energy generation due to stimulated and spontaneous emission. The drain term includes the different losses: emission (modal loss) 3.59 and bulk absorption loss 3.60. Emission (modal loss) accounts for the loss in the mode which relies on the length of the cavity, $L$, and the reflectivity of the ends of the cavity, $R_{1}$ and $R_{2}$. Bulk absorption loss accounts for re-absorption of the photons into an undesired energy gap and relies on the extinction coefficient, $\kappa$, and wave number in free space, $\lambda_{0}$. The photon lifetime, (3.61), derives from the total absorption length, $\alpha_{m}+\alpha_{a}$, and effective velocity through the device, $\frac{c}{n_{e f f}}$. The photon rate equation describes energy conservation for a single wavelength and cavity mode of that wavelength in the device. The optical density derived from the Helmholtz equation Sec. 3.4 provides the cavity effects with the material gain Sec. 3.3.2 generates the net modal stimulated emission in 3.62. Each net modal stimulated emission term must be included in the carrier equations.

$$
\frac{\partial U}{\partial t}=-\nabla \cdot \vec{u}-\vec{E} \cdot \vec{J}
$$




$$
\begin{gathered}
\alpha_{m}=\frac{1}{2 L} \ln \left(\frac{1}{R_{1} R_{2}}\right) \\
\alpha_{a}=\frac{\int \frac{4 \pi \kappa}{\lambda_{0}}\|E\|^{2}}{\int\|E\|^{2}} \\
\frac{1}{\tau_{p h}}=\frac{c}{n_{e f f}}\left(\alpha_{m}+\alpha_{a}\right) \\
\frac{\partial S}{\partial t}=-\frac{S}{\tau_{p h}}+B_{r}\left(n p-n_{i}^{2}\right)+\frac{c}{n_{e f f}} G_{m} S
\end{gathered}
$$

\subsubsection{Emitted Power}

Power output of the cavity derives from photon loss due to emission. Utilizing the

loss term, $\frac{S}{\tau_{p h}}$, and multiplying by the energy of each photon, $h f$, yields the power loss of the cavity. To obtain the cavity's emitted power, the power loss multiplied the emitted percentage yields (3.63) [23]. This component marks the extent of base models necessary to simulate basic electrical and optical device operation.

$$
P=\frac{\alpha_{m}}{\alpha_{m}+\alpha_{a}} h f \frac{S}{\tau_{p} h}
$$

The construction, discretization, and computation of the Poisson, Current Continuity, Helmholtz, and Photon Rate equations occur utilizing Python. The next section, Sec. 4, shows the methodologies used to construct and solve these equations and their constituent models. 
Chapter 4

\section{DEVELOPING IN PYTHON}

The models described in Sec. 3 constitute the information that needs solving. The actual model implementation occurs utilizing the DEVSIM Solver or the 1-D Helmholtz Solver. The high-level script executes each solver individually and combines the results to provide all the desired information from the models specified above. Appendix A provides links to the DEVSIM manual, DEVSIM source code, and Simulator source code.

\subsection{DEVSIM Solver}

As noted in Sec. 2.3, DEVSIM constructs the Control Volume Equation (2.1) utilizing the node, edge, and element models. A user-defined equation solves for a special implementation of a node model known as a node solution which updates all other dependent models after convergence. DEVSIM's internal matrix constructor assumes that all designated model descriptions were generated with the appropriate sign for a left hand side equation. Furthermore, a model of a given type may be described by a subsidiary model of the same type, constants, and provided mathematical functions. To cross model types, DEVSIM provides in their API a list of up-converting functions with a node solution providing the base of any future model. DEVSIM also provides methods to define additional mathematical functions from their python interface or by direct implementation in the source code. DEVSIM processes the constructed equations and produces a matrix and its Jacobian to converge upon a solution uti- 
lizing the Newton method. DEVSIM provides several solving methods including DC, Transient DC, AC, and Noise.

\subsubsection{Material Parameters And Units}

This project utilized OC1C10-PPV as the main organic material and retrieved the material parameters for OC1C10-PPV from [19], [3], [29], [30] including refractive index, band edges, constant mobilities, permittivity, constant bi-molecular recombination rate, and gain. The other materials utilized by this project include Indium Tin Oxide and Calcium for the contacts. The parameters needed for these materials included refractive index obtained from [31] and work function obtained from [4]. The units for these parameters should all be in the standard SI units except for meters and Joules as the simulator assumes centimeters and Electron-Volts. DEVSIM provides a base implementation of a database to store these values and access them in the models. The data entries in the database include the name of the material, the material parameter name, the value of the parameter, its corresponding units, and a generic description of the parameter. Upon generating the mesh, each region gains a material type that corresponds to an entry in the database. If a parameter value does not exist for a given material, the generic global material should contain the value of this parameter or an error occurs.

\subsubsection{Python Model Format}

The project developer built three library files: util/model.py, util/model_create.py, and util/model_factory.py to create an infrastructure that allows model additions to the simulator. These libraries provide a simple model format demonstrated in Listing 4.1 to create new models. 


\section{Listing 4.1: Model Format}

class NetDoping( NodeModel):

$$
\begin{aligned}
& \text { def _-_init_-( self, device, region ): } \\
& \text { self._name }=(\text { self } \cdot \operatorname{get} N a m e(),) \\
& \text { self ._equations }=\left(" \mathrm{Nd}_{\llcorner}-{ }_{\lrcorner} \mathrm{Na} ",\right) \\
& \text { self._solution Variables }=(\text { ) } \\
& \text { self . parameters }=\left\{\text { "Nd" : "Donor }{ }^{\text {Concentration" }}\right. \text {, } \\
& \text { "Na" : "Acceptor } \leftarrow \text { Concentration" }
\end{aligned}
$$

super(NetDoping, self).generateModel(device, region)

The requirements include the name of the model and the corresponding expression. These components must be entered as lists even if they constitute only a single item. The solutionVariables allow for derivation of the expression with respect to that variable. The parameters aid the user in remembering other constants and parameters necessary for operation. The most important aspect of the format relies on inheriting from one of the three model types in util/model.py as seen by (NodeModel). Furthermore, these library files simplified most of the API provided by DEVSIM, however, the DEVSIM API should be learned for generating new boundary conditions as these implementations tend to rely on more specific information. These new models must be instantiated in the overall script, see Listing 4.2 .

\section{Listing 4.2: Equation Builder}

\#MODEL INSTANTIATION

potential. ElectricField (device, region)

potential. SemiconductorIntrinsicCarrierPotential (device, region)

\#EQUATION CONSTRUCTION (needs device, region, Node Solution Variable) 


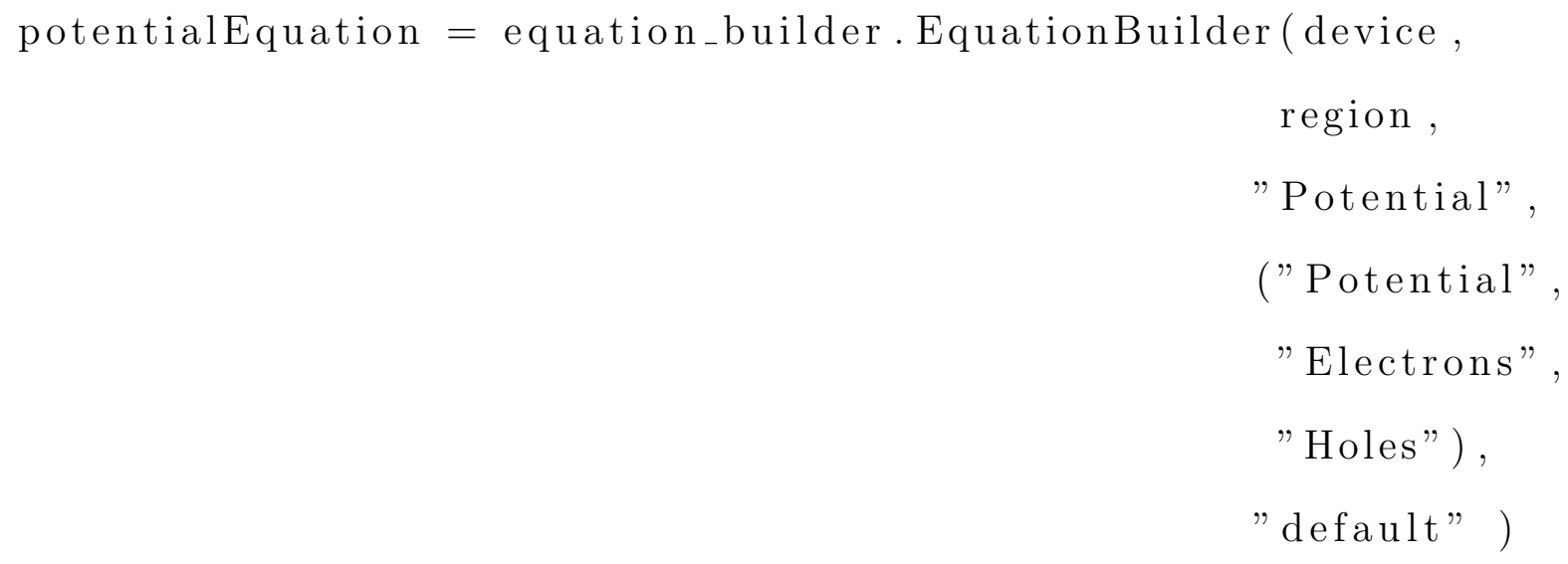

\#MODEL ADDITION TO EQUATION

potentialEquation .addModel ("PotentialEdgeFlux" , "EdgeModel") potentialEquation .addModel("PotentialIntrinsicCharge" , "NodeModel") \#BUILD EQUATION

potentialEquation . buildEquation()

\subsubsection{Equation Building}

An equation for each solution variable (intrinsic potential; electron, hole, and photon densities) forms from the instantiated models. The project developer constructed a library file equation_builder.py to manage the addition of new models to an equation and build the equation on the region as seen in Listing 4.2 As stated above, Node Solutions represent the base models, which all other models rely upon and only the user or an evaluated equation sets the values of these models. All other models update after their parent model updates. For this reason, the user must instantiate the models and add them to an equation for proper evaluation. 


\subsubsection{Contact Assembly}

The boundary conditions in Sec. 3 with the junctions including metal assume a perfect, isotropic conductor and represent the end of the computation domain aside from boundary conditions produced exclusively to end the computation domain. The simulation of a perfect, isotropic conductor does not offer any additional information in the current system than the boundary conditions specified. Furthermore, the simulation of these components would extend the computation domain requiring more processing time to converge upon a solution.

\subsection{Helmholtz Solver}

The 1-D Helmholtz Solver calculates the effects of the cavity on wave propagation and the resulting optical density through the region. The separation of these computations from the DEVSIM Solver results from the lack of implementation of complex numbers in the solver and the different types of solution methods needed to calculate the waveguide refractive index and optical density.

\subsubsection{Waveguide Refractive Index}

The waveguide refractive index, $n_{\text {eff }}$, as stated in Sec. 3.4, represents the wave propagation index that occurs from many-layered materials of varying refractive indices. Two equations that equate the optical density and derivative of the optical density result from the interface from two differing refractive indices materials, see Fig. C.1 in Appendix C. This generation of equations results in $2(N+1)$ equations, see Fig. C.2 in Appendix C. The matrix determinant of this system of equations yields a transcendental equation as shown in Listing 4.3. The solutions to this transcenden- 
tal equation represent the waveguide modes. This portion of the Helmholtz solver currently utilizes the fundamental Transverse Electric mode.

\section{Listing 4.3: Transcendental Equation}

def transcendental $(\mathrm{x})$ :

\#2N+2 EQUATIONS

$\operatorname{length}=2 * \operatorname{len}($ get_region_list $($ device=self__device $))+2$

\#COMPLEX MATRIX

modalMatrix $=$ np.zeros ((length, length $),$ dtype='complex')

self ._refractiveIndices. sort (key=lambda $x: \quad x[0], \quad$ reverse=True)

for index, indices in enumerate( self. _refractiveIndices ):

position $=$ indices $[0]$

\#CONTACTS SHOULD HAVE DECAYING WAVES

boundTypes $=$ indices $[3]$

if boundTypes = "Contact":

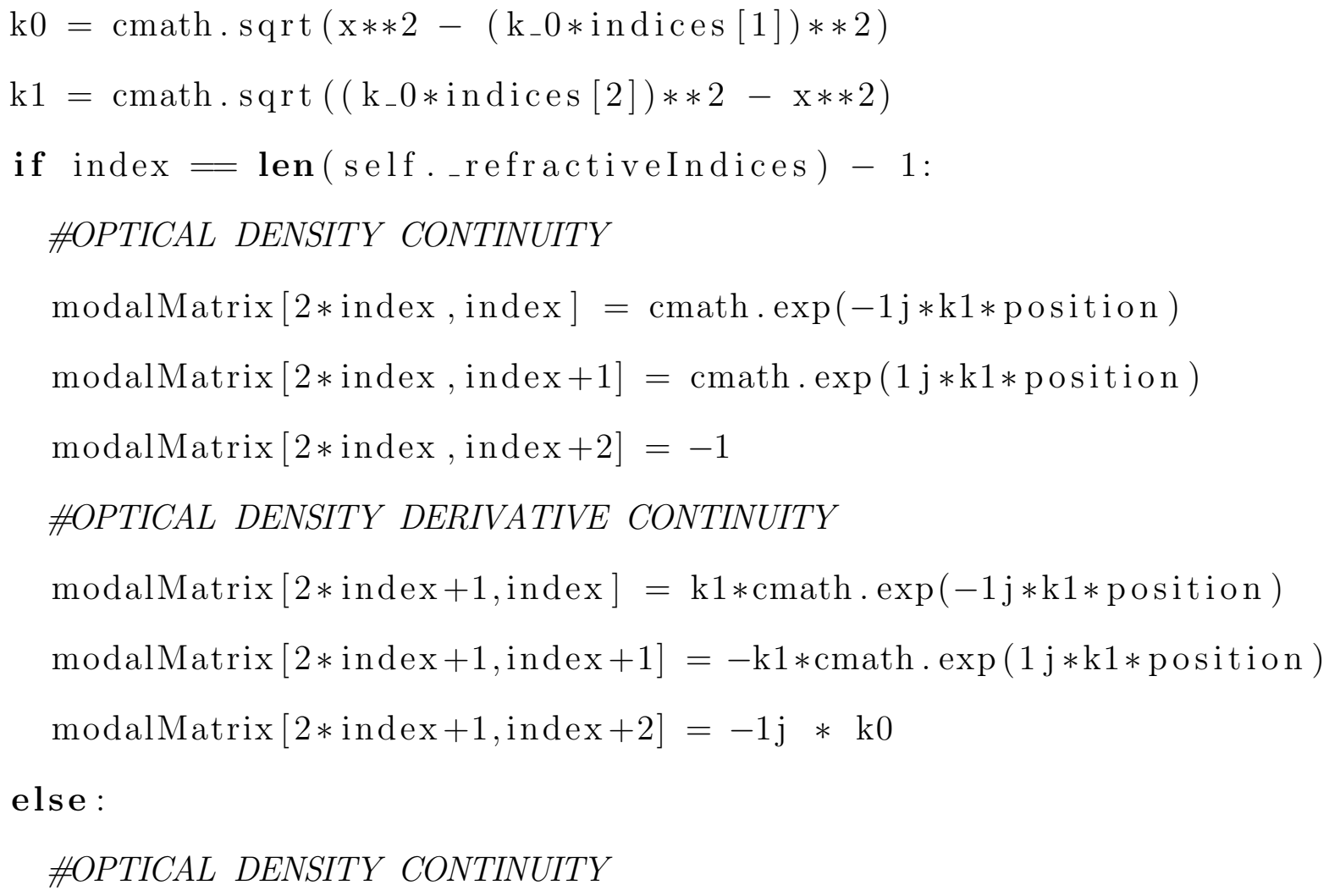


modalMatrix $[2 *$ index, index $]=-1$

modalMatrix $[2 *$ index, index +1$]=$ cmath $\cdot \exp (-1 \mathrm{j} * \mathrm{k} 1 *$ position $)$

modalMatrix $[2 *$ index, index +2$]=$ cmath $\cdot \exp (1 \mathrm{j} * \mathrm{k} 1 *$ position $)$

\#OPTICAL DENSITY DERIVATIVE CONTINUITY

modalMatrix $[2 *$ index +1, index $]=1 \mathrm{j} * \mathrm{k} 0$

modalMatrix $[2 *$ index +1, index +1$]=\mathrm{k} 1 * \operatorname{cmath} . \exp (-1 \mathrm{j} * \mathrm{k} 1 *$ position $)$

modalMatrix $[2 *$ index +1, index +2$]=-\mathrm{k} 1 * \operatorname{cmath} . \exp (1 \mathrm{j} * \mathrm{k} 1 *$ position $)$

\#INTERFACES SHOULD HAVE PROPAGATING WAVES

elif boundTypes $=$ "Interface" :

$\mathrm{k} 0=$ cmath. $\operatorname{sqrt}\left(\left(\mathrm{k}_{-} 0 *\right.\right.$ indices $\left.\left.[1]\right) * * 2-\mathrm{x} * * 2\right)$

$\mathrm{k} 1=$ cmath. sqrt $\left(\left(\mathrm{k}_{-} 0 * \mathrm{indices}[2]\right) * * 2-\mathrm{x} * * 2\right)$

\#OPTICAL DENSITY CONTINUITY

modalMatrix $[2 *$ index, index $]=$ cmath. $\exp (-1 \mathrm{j} * \mathrm{k} 0 *$ position $)$

modalMatrix $[2 *$ index, index +1$]=$ cmath $\exp (1 \mathrm{j} * \mathrm{k} 0 *$ position $)$

modalMatrix $[2 *$ index, index +2$]=$ cmath. $\exp (-1 \mathrm{j} * \mathrm{k} 1 *$ position $)$

modalMatrix $[2 *$ index, index +3$]=$ cmath $\cdot \exp (1 \mathrm{j} * \mathrm{k} 1 *$ position $)$

\#OPTICAL DENSITY DERIVATIVE CONTINUITY

modalMatrix $[2 *$ index, index $]=-1 \mathrm{j} * \mathrm{k} 0 *$ cmath. $\exp (-1 \mathrm{j} * \mathrm{k} 0 *$ position $)$

modalMatrix $[2 *$ index, index +1$]=1 \mathrm{j} * \mathrm{k} 0 * \operatorname{cmath} \cdot \exp (1 \mathrm{j} * \mathrm{k} 0 *$ position $)$

modalMatrix $[2 *$ index, index +2$]=-1 \mathrm{j} * \mathrm{k} 1 * \operatorname{cmath} \cdot \exp (-1 \mathrm{j} * \mathrm{k} 1 *$ positin $)$

modalMatrix $[2 *$ index, index +3$]=1 \mathrm{j} * \mathrm{k} 1 * \operatorname{cmath} \cdot \exp (1 \mathrm{j} * \mathrm{k} 1 *$ position $)$

else :

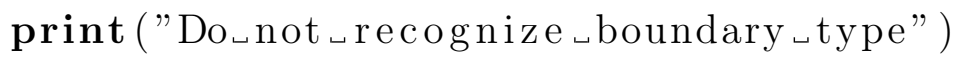

return ( scipy.linalg.det(modalMatrix) * $1 \mathrm{j})$.real 


\subsubsection{Optical Density}

The transverse optical density, $E_{w}$, results from the complex eigen-value analysis of the Helmholtz equation. Applying the Helmholtz equation to each node in the mesh results in a system of equations. The discretization of the $2^{\text {nd }}$ order $\nabla$ operator in $1-D$ comes from [32] which constitutes the values of the entries in the matrix as seen in Listing 4.4. The eigen-vectors of the resulting matrix generated from the discretized Helmholtz equation applied to each node yield the transverse optical density of the device. The lowest energy eigen-value determines the fundamental optical mode of the region and yields the fundamental eigen-vector utilized by DEVSIM upon the next iteration.

\section{Listing 4.4: Matrix Construction}

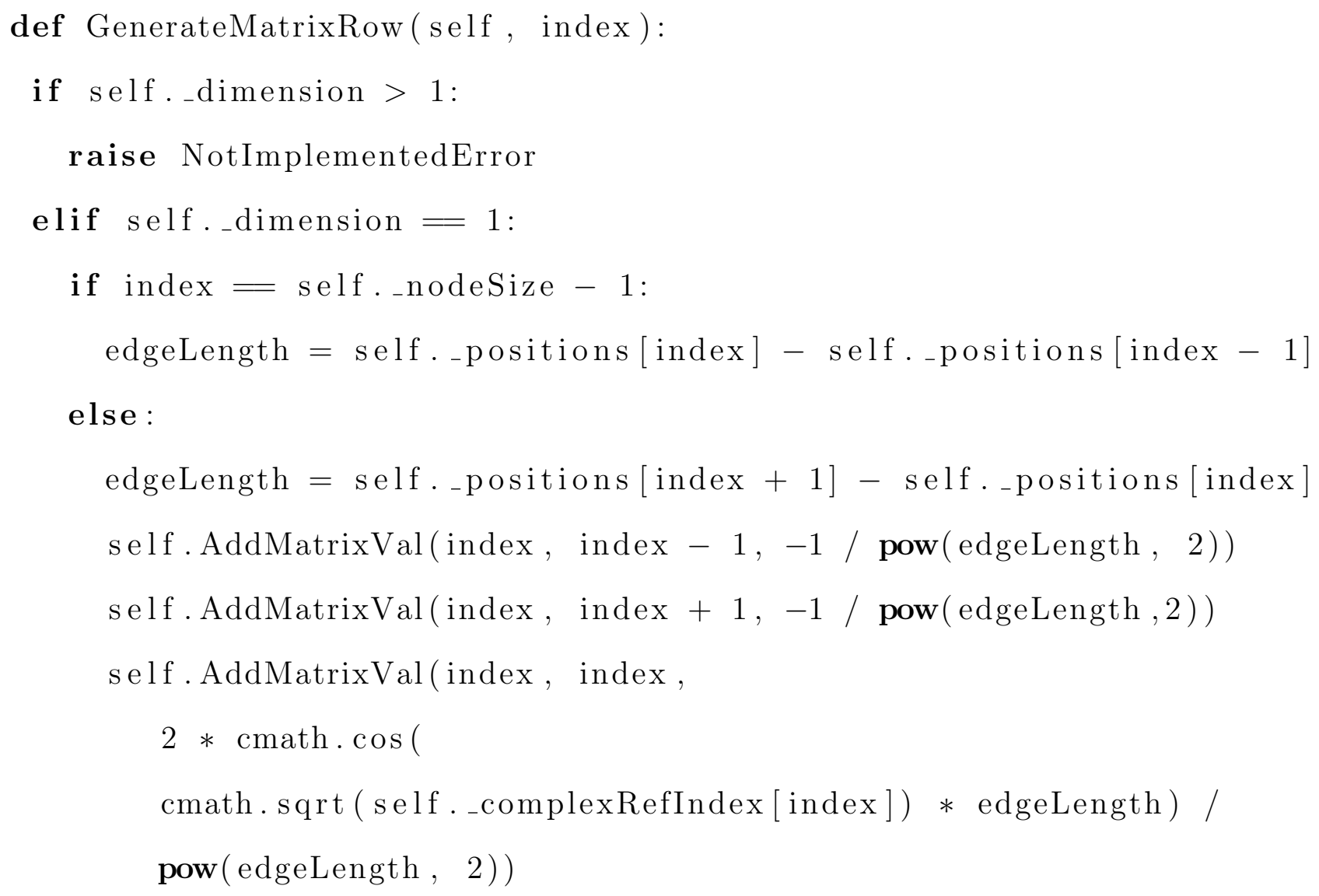




\subsection{Semiconductor Simulation}

An overall script, $1 D_{-} P P V \_$diode.py generates the mesh, constructs the necessary models and equations in DEVSIM, initializes the Helmholtz Solver, and iteratively solves the equations presented in Sec. 3 with the DC condition as shown in Fig. 4.1.

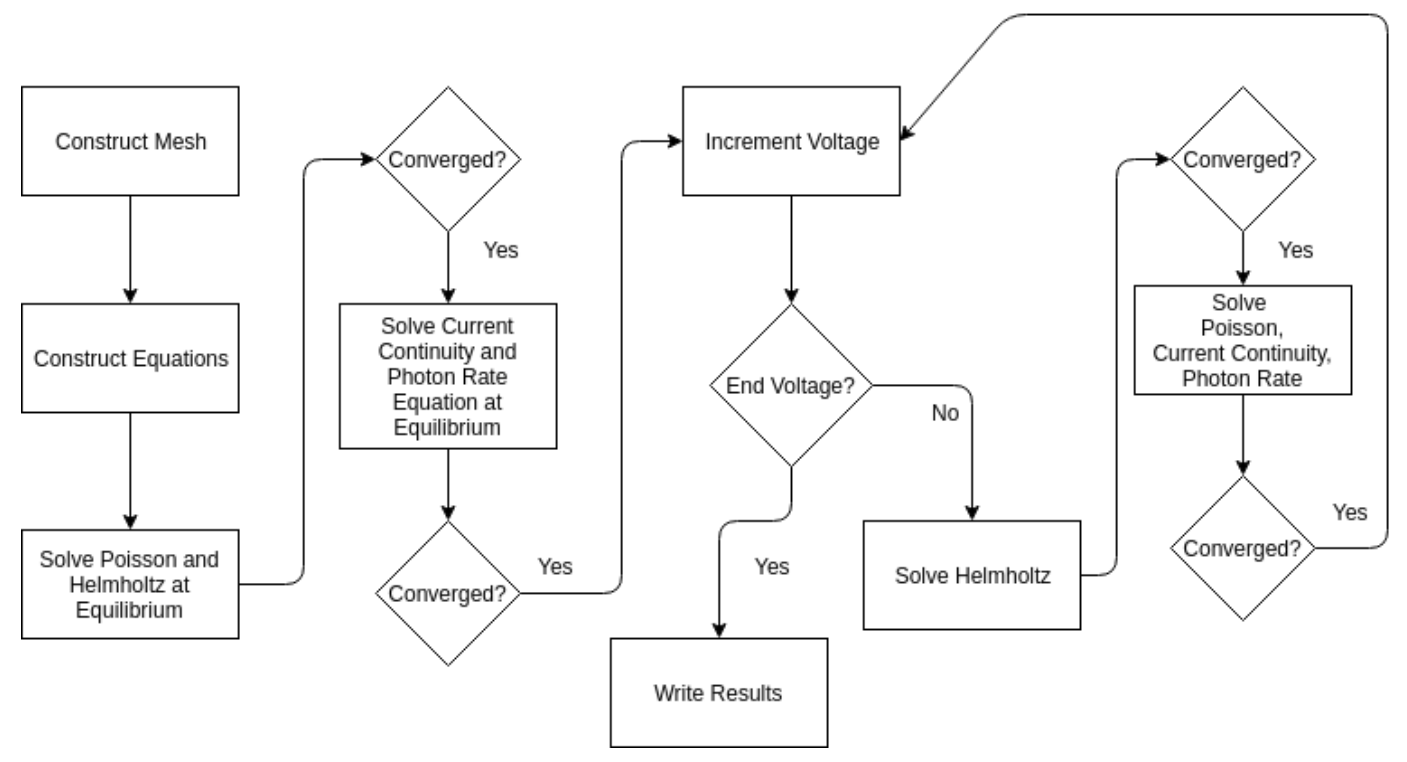

Figure 4.1: Full Execution Cycle of Simulator in 1D_PPV_diode.py

The mesh subroutine resides in mesh.py and constructs a 1D mesh with the appropriate number of nodes and spacing between the nodes. The equation subroutine differs for each equation, but follow the format shown in Listing 4.2 where the script initializes the models and dependent equations for each region. The solver then executes in two major steps: equilibrium and bias application. The equilibrium stage calculates the intrinsic potential, equilibrium carrier concentrations, and the optical density of the cavity. The bias application stage utilizes these evaluated models to initialize the carrier concentrations described by the current continuity and Helmholtz equations. The voltage sweep occurs after this initialization by setting the voltage parameter at the contact at each iteration and executing the solver. The execution of the DEVSIM 
and Helmholtz solver occur utilizing the solve commands as shown in Listing 4.5. The DEVSIM solver evaluates the Poisson, Current Continuity, and Photon Rate equation and then the Helmholtz solver executes for each applied voltage reporting the absolute and update errors for each iteration of the Newton Method. DEVSIM writes the result of the last converged iteration to a file allowing for voltage, current, carrier density, optical gain, and luminescence.

\section{Listing 4.5: Solver Executions}

$$
\begin{aligned}
& \text { solve }(\mathbf{t y p e}=" \mathrm{dc} ", \text { absolute_error }=1 \mathrm{e} 06, \\
& \text { relative_error }=1 \mathrm{e}-06, \text { maximum_iterations }=30) \\
& \text { opticalDensity }=\text { helmholtz. HelmholtzSolver (device, "5e14", "MyRegion") } \\
& \text { opticalDensity.Solve () }
\end{aligned}
$$


Chapter 5

\section{RESULTS AND DISCUSSION}

The project constructs the simulation utilizing a 1-D equidistant mesh with ITO and Calcium contacts encasing the 200nm long OC1C10-PPV polymer semiconductor as shown in Fig. 5.1. In the OC1C10-PPV, the solver calculates the potential, electron, hole, and photon densities from the Poisson, Current Continuity, and Photon Rate equations. These node solutions then propagate their values to dependent models such as current and photon emission. The results below show solution densities, I-V characteristics and optical output for varying combinations of models: Ohmic vs.

Schottky Boundary Conditions and Constant vs. Field-Dependent Mobility. This section focused on the convergence of the simulator starting with the simplest case of Constant Mobility and Ohmic Contacts. With each instance, the simulator includes more complex models until the Field-Dependent Mobility and Schottky Contacts instance converges. These two models represent a more realistic Organic LED. The Field-Dependent Mobility marks the main difference between traditional and organic semiconductors other than the material parameter values; and the Schottky Contacts represent more realistic metal-semiconductor junctions.

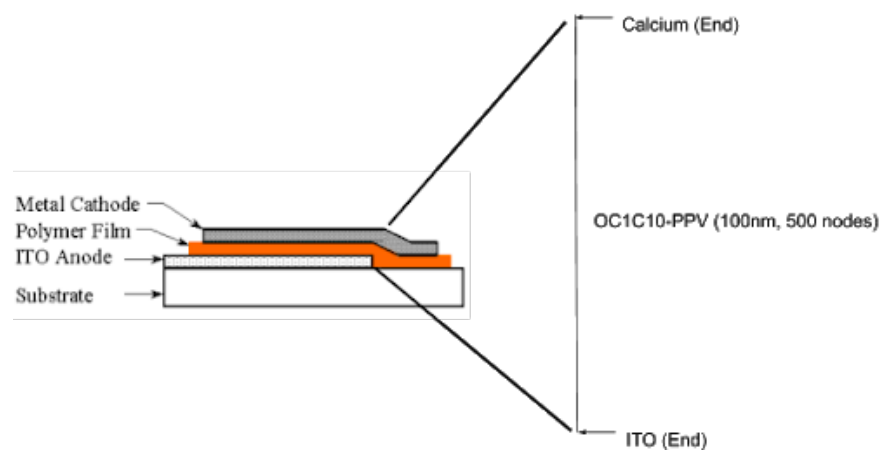

Figure 5.1: LED Device Structure and Mesh Representation in Simulator 
Table 5.1: Simulation Parameters

\begin{tabular}{|c|c|}
\hline Parameter & Value \\
\hline Number of Nodes & 500 \\
\hline Node Spacing & $3 \mathrm{e}-11 \mathrm{~m}$ \\
\hline Simulation Dimension & $1-\mathrm{D}$ \\
\hline N-Type Contact & Calcium \\
\hline Bulk & OC1C10-PPV \\
\hline P-Type Contact & ITO \\
\hline Voltage Increment & $.1 \mathrm{~V}$ \\
\hline
\end{tabular}

Table 5.2: Global Constants

\begin{tabular}{|c|c|c|c|}
\hline Name & Value & Units & Description \\
\hline ElectronCharge & $1.602 \mathrm{e}-19$ & Coulombs & Charge of an Electron \\
\hline $\mathrm{T}$ & 300 & Kelvin & Temperature \\
\hline $\mathrm{k}$ & $8.6173303 \mathrm{e}-05$ & $\mathrm{eV} / \mathrm{K}$ & Boltzmann Constant \\
\hline Permittivity & $8.85 \mathrm{e}-14$ & $\mathrm{~F} / \mathrm{cm}^{2}$ & Vacuum Permittivity \\
\hline $\mathrm{h}$ & $4.135 \mathrm{e}-15$ & $\mathrm{eV}^{*} \mathrm{~s}$ & Planck's Constant \\
\hline $\mathrm{pi}$ & 3.1415 & $\mathrm{~N} / \mathrm{A}$ & $\pi$ \\
\hline $\mathrm{c}$ & 299000000.0 & $\mathrm{~m} / \mathrm{s}$ & speed of light \\
\hline
\end{tabular}


Table 5.3: OC1C10-PPV Material Parameters

\begin{tabular}{|c|c|c|c|}
\hline \\
\hline Name & Value & Units & Description \\
\hline $\mathrm{B}$ & $2.9 \mathrm{e}-05$ & $\mathrm{eV}(\mathrm{m} / \mathrm{V})$ & Empirical Mobility Constant \\
\hline $\mathrm{C}$ & $3 e-05$ & $(\mathrm{~m} / \mathrm{V}) \cdot 5$ & site spacing \\
\hline Permittivity & $2.6550000000000003 \mathrm{e}-13$ & $\mathrm{~F} / \mathrm{cm}^{2}$ & OC1C10 Permittivity \\
\hline $\mathrm{a}$ & $1.2 \mathrm{e}-07$ & $\mathrm{~cm}$ & lattice spacing \\
\hline eps_r & 3 & & Relative Permittivity \\
\hline sigma & 0.112 & $\mathrm{eV}$ & energetic disorder bandwidth \\
\hline B_r & $3.32 \mathrm{e}-11$ & $\mathrm{~cm}^{3} / \mathrm{s}$ & bimolecular recombination rate \\
\hline $\mathrm{Na}$ & 0 & $1 / \mathrm{cm}^{3}$ & acceptor ions \\
\hline $\mathrm{Nd}$ & 0 & $1 / \mathrm{cm}^{3}$ & donor ions \\
\hline mu_inf_n & $5.1 \mathrm{e}-06$ & $\mathrm{~cm}^{2} / V s$ & Field-Dependent Electron mobility \\
\hline mu_inf_p & $5.1 \mathrm{e}-05$ & $\mathrm{~cm}^{2} / V s$ & Field-Dependent Hole mobility \\
\hline mu_n & $5 \mathrm{e}-06$ & $\mathrm{~cm}^{2} /(V s)$ & Constant Electron Mobility \\
\hline mu_p & $5 \mathrm{e}-05$ & $\mathrm{~cm}^{2} /(V s)$ & Constant Hole Mobility \\
\hline E_Electrons & -2.8 & $\mathrm{eV}$ & conduction band energy (LUMO) \\
\hline E_Holes & -4.9 & $\mathrm{eV}$ & valence band energy (HOMO) \\
\hline N_Electrons & $2.5 \mathrm{e} 19$ & $1 / \mathrm{cm}^{3}$ & conduction band carriers \\
\hline N_Holes & $2.5 \mathrm{e} 19$ & $1 / \mathrm{cm}^{3}$ & valence band carriers \\
\hline M_Electrons & $9.0864 \mathrm{e}-35$ & $\mathrm{~kg}$ & mass of electrons \\
\hline M_Holes & $9.0864 \mathrm{e}-35$ & $\mathrm{~kg}$ & mass of holes \\
\hline $\mathrm{k}_{-} 5 \mathrm{e} 14$ & 0.02 & $\mathrm{~N} / \mathrm{A}$ & Extinction Coefficient of $5 \mathrm{e} 14 \mathrm{~Hz}$ \\
\hline n_5e14 & 1.96 & $\mathrm{~N} / \mathrm{A}$ & Refractive Index at $5 \mathrm{e} 14 \mathrm{~Hz}$ \\
\hline gain0_5e14 & 2000 & $1 / \mathrm{cm}$ & Intrinsic gain \\
\hline N_t & $1.1 \mathrm{e}+17$ & $1 / \mathrm{cm}^{3}$ & Density of Trap States \\
\hline
\end{tabular}

Table 5.4: ITO Material Parameters

\begin{tabular}{|c|c|c|c|}
\hline Name & Value & Units & Description \\
\hline WorkFunction & -4.7 & $\mathrm{eV}$ & Work Function \\
\hline $\mathrm{k} \_5 \mathrm{e} 14$ & 0.0023 & $\mathrm{~N} / \mathrm{A}$ & extinction coefficient at $5 \mathrm{e} 14 \mathrm{~Hz}$ \\
\hline $\mathrm{n} \_5 \mathrm{e} 14$ & 1.72 & $\mathrm{~N} / \mathrm{A}$ & refractive index at $5 \mathrm{e} 14 \mathrm{~Hz}$ \\
\hline
\end{tabular}

Table 5.5: Calcium Material Parameters

\begin{tabular}{|c|c|c|c|}
\hline Name & Value & Units & Description \\
\hline WorkFunction & -2.9 & $\mathrm{eV}$ & Calcium Work Function \\
\hline k_5e14 & 2.6362 & N/A & extinction coefficient \\
\hline n_5e14 & 0.29 & N/A & refractive index \\
\hline
\end{tabular}




\subsection{Density Distributions}

Fig. 5.2 shows the nodal values of the electrostatic potential, $\psi=E_{f}-E_{i}$, through the material at equilibrium. The intrinsic Fermi level marks the reference point for the electrostatic potential. For both cases, $x=0$ represents the n-type contact and $x=1 e-7$ represents the p-type contact. Fig. 5.2 demonstrates the difference between the net bandgaps between Ohmic and Schottky contacts. The Ohmic contacts case results in a net gap of $2.1 \mathrm{eV}$ between the electron and hole quasi-Fermi levels or the full bandgap of OC1C10 whereas the Schottky contacts case results in a reduced gap of $1.9 \mathrm{eV}$ between the quasi-Fermi levels.

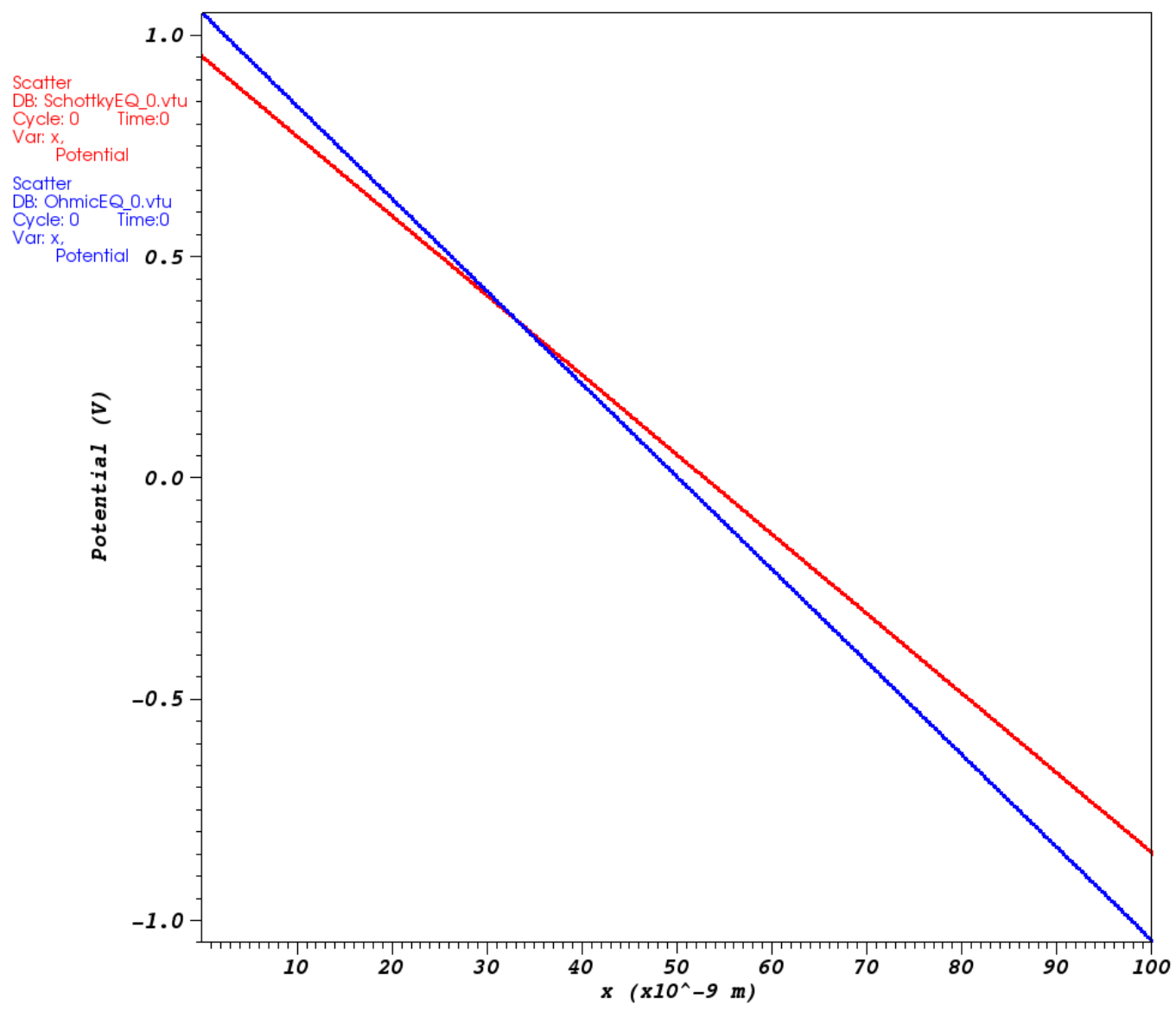

Figure 5.2: Nodal Intrinsic Potential for Ohmic and Schottky Contacts 
The additional barrier height in the Schottky contacts case yields a lower electrostatic potential and consequently lower electron and hole densities shown in Figures 5.3 and 5.4 .

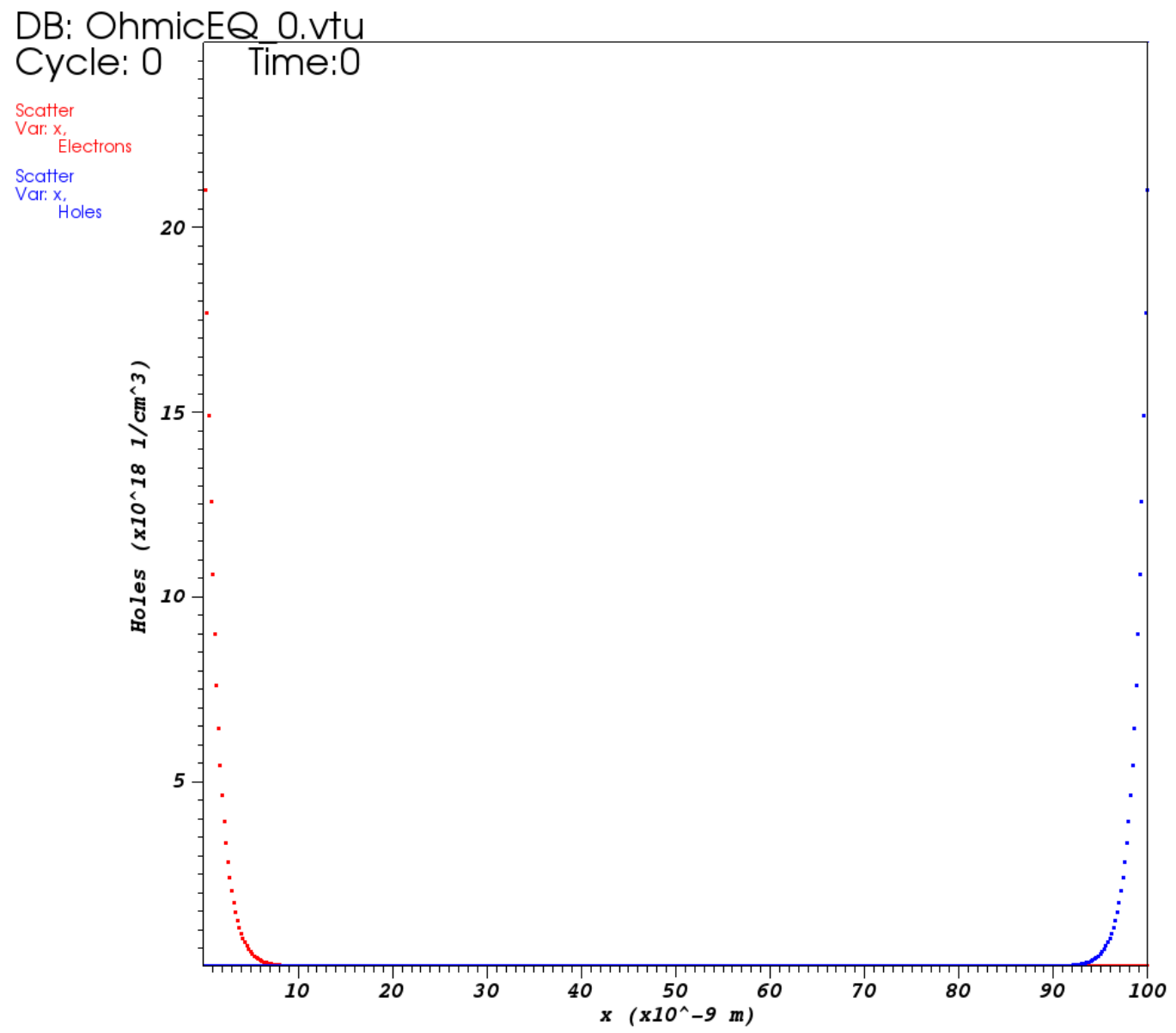

Figure 5.3: Nodal Electron and Hole Densities for Ohmic Contacts 


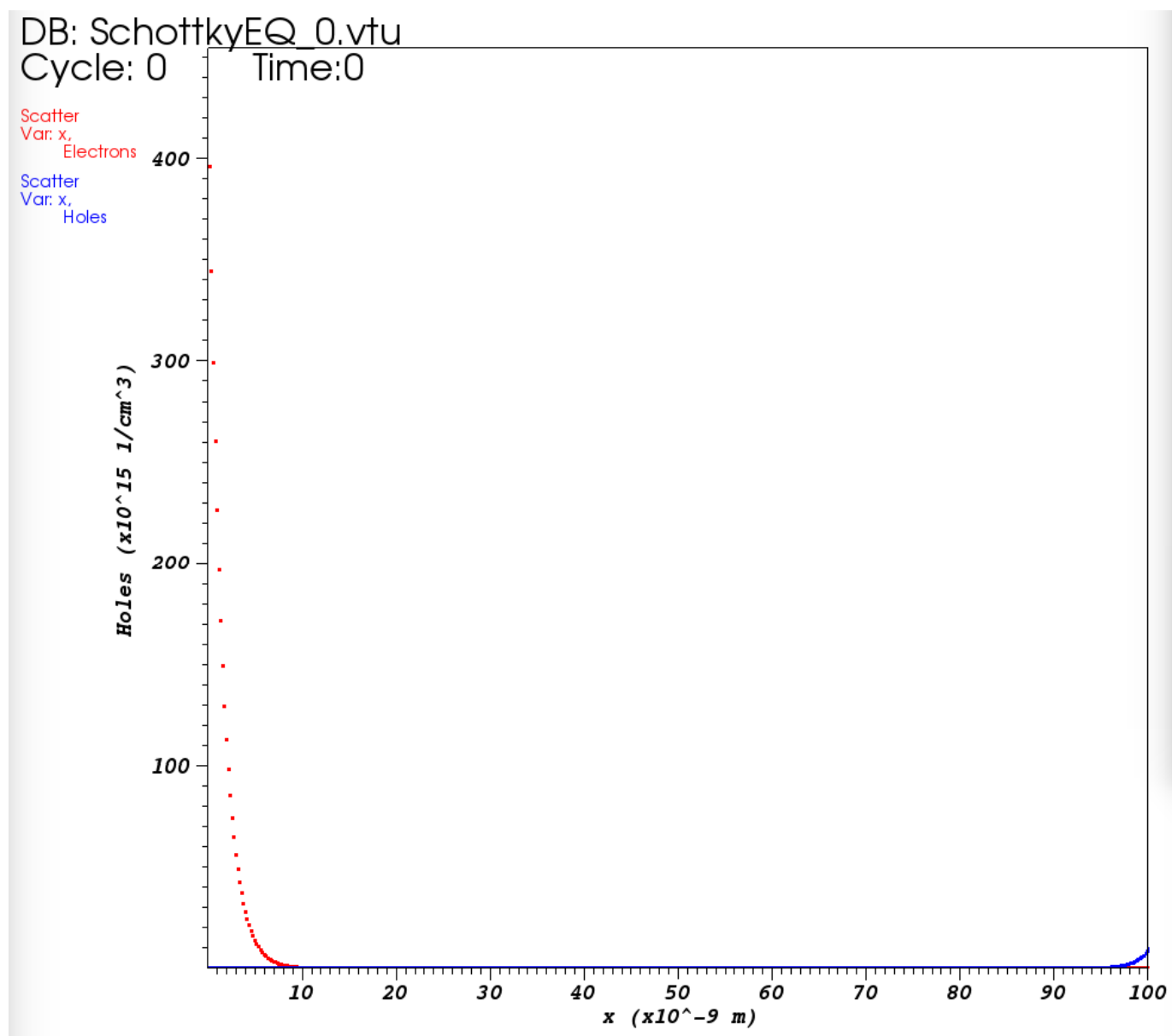

Figure 5.4: Nodal Electron and Hole Densities for Schottky Contacts 


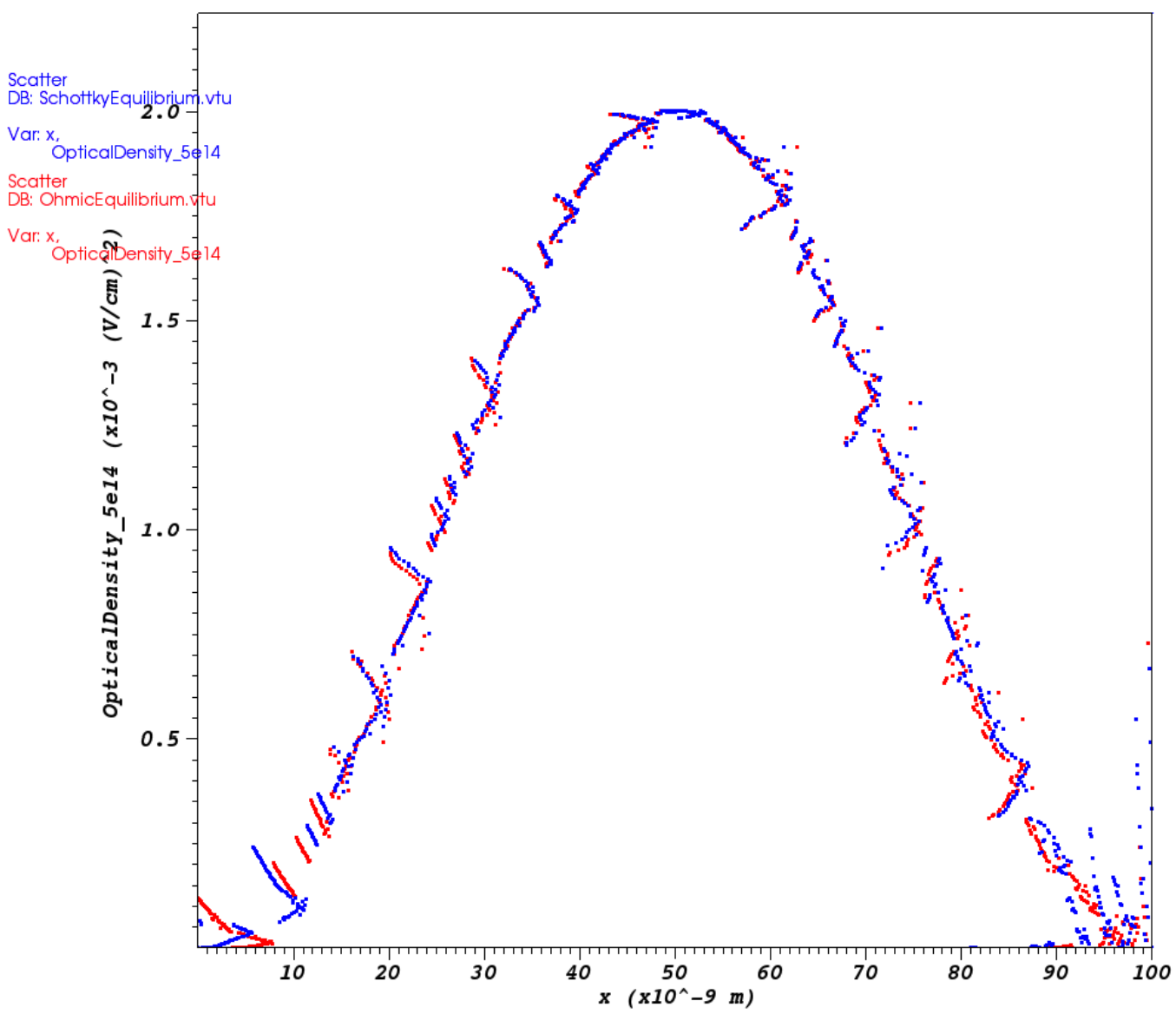

Figure 5.5: Nodal Optical Densities for Ohmic and Schottky Contacts for Contact Refractive Indices of 1

Fig. 5.5 shows the modal profile of the LED structure with metal contact refractive indices of 1 and a 1000 node mesh. This profile confirms the convergence of the Helmholtz Effective Index and Optical Density solvers with sufficient discretization of the mesh. The fluctuations within the magnitude of the densities derive from the optical gain. The optical gain nonlinearly relies on the electron and hole populations which also derive from the nonlinear Scharfetter-Gummel method. Despite the nonlinearities, the optical density shows the distribution associated with a wave in its fundamental mode. To improve computational efficiency, the simulation instances 
below utilized a 500 node mesh. However, the modal profile affects optical gain which may improve the results of the lasing section, 6 .

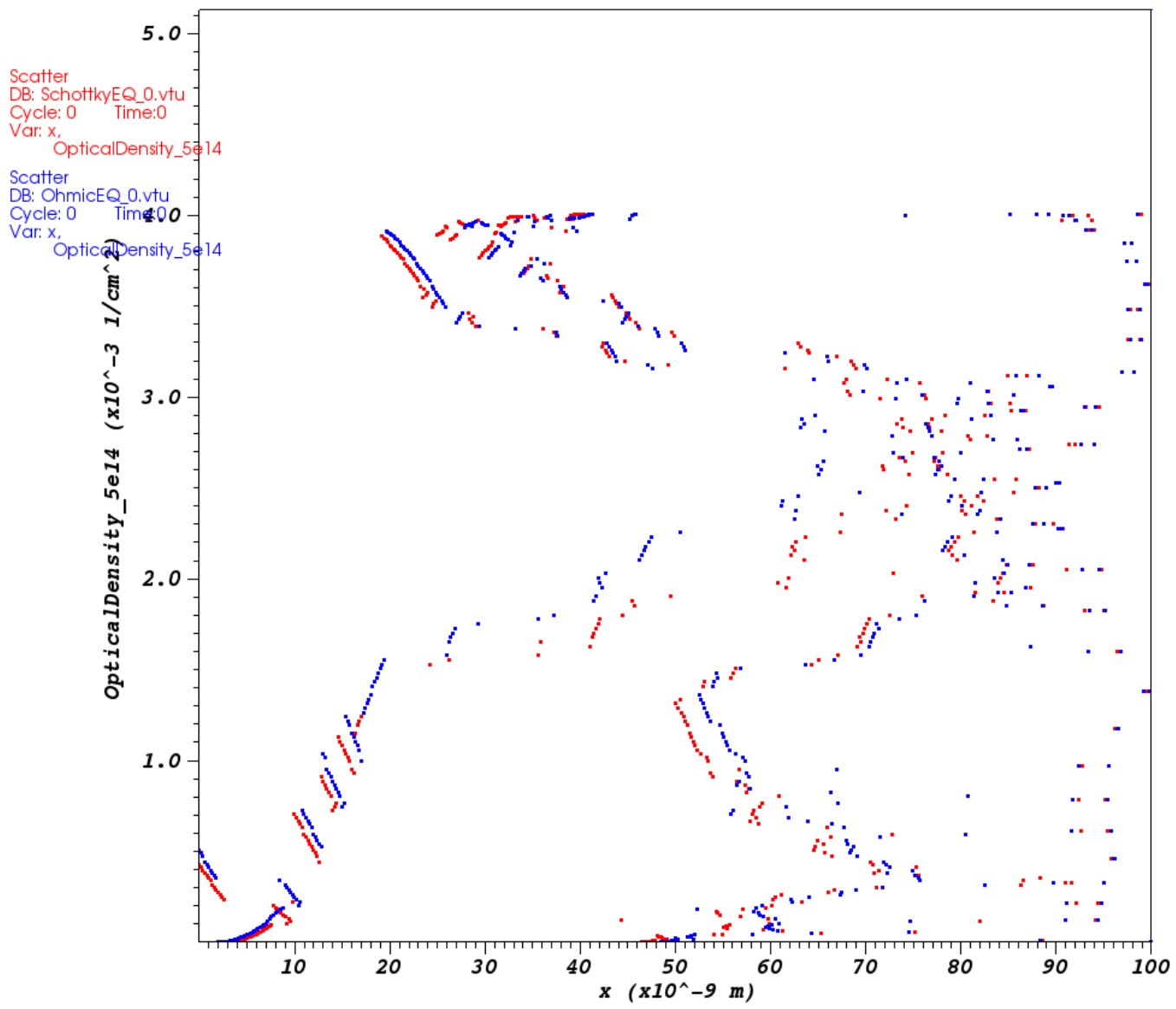

Figure 5.6: Nodal Optical Densities for Ohmic and Schottky Contacts for Calcium and ITO Refractive Indices

Fig. 5.6 shows the resulting optical density distributions from the Ohmic and Schottky contact cases with the 500 node mesh. The 500 node mesh does not provide enough discretization to achieve the fundamental mode. For instances testing optical gain, a finer optical mesh is necessary. 


\section{$5.2 \quad$ I-V Curves}

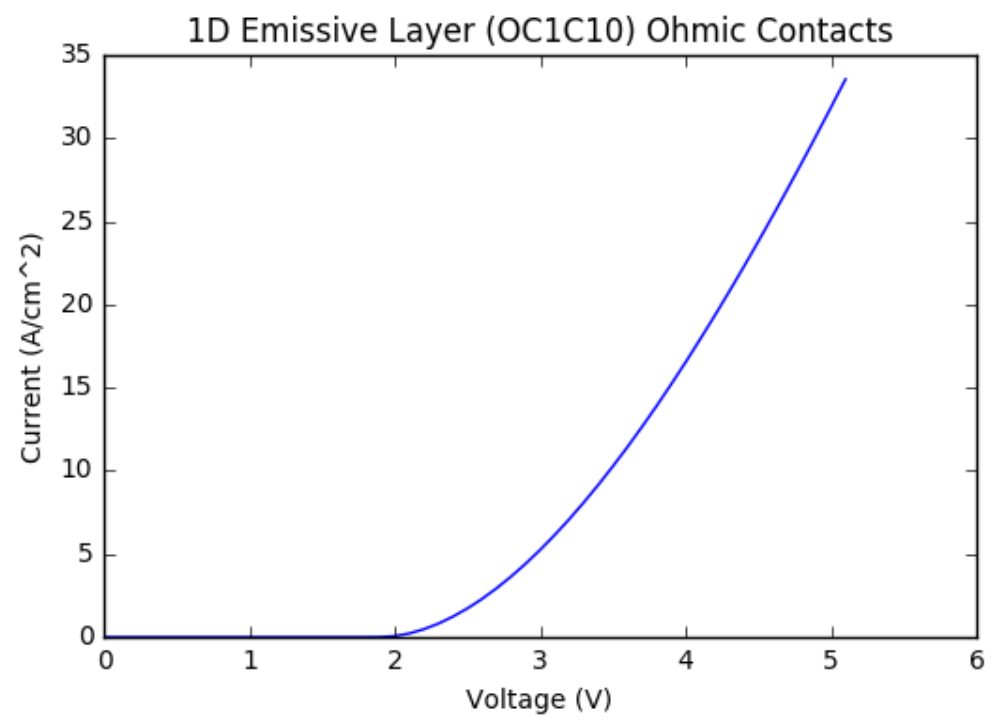

Figure 5.7: I-V Plot of 1D Emissive Layer and Ohmic Contacts

Fig. 5.7 demonstrates typical LED operation with a turn-on voltage at the bandgap of the material for $\mathrm{OC} 1 \mathrm{C} 10,2.1 \mathrm{eV}$. The nonlinearity that occurs above the turnon voltage in this plot results from the Ohmic contact boundary condition. The Ohmic boundary condition specifies the electron and hole populations at the boundary allowing the bulk current to dominate the current flow through the device. The bulk current relies on the nonlinear Scharfetter-Gummel method to discretize the current flow on an edge in the device. Finally, the scale of the bulk current demonstrates the lower mobilities existent in organic materials, $10^{-5}-10^{-7} \frac{\mathrm{cm}^{2}}{V s}$. 


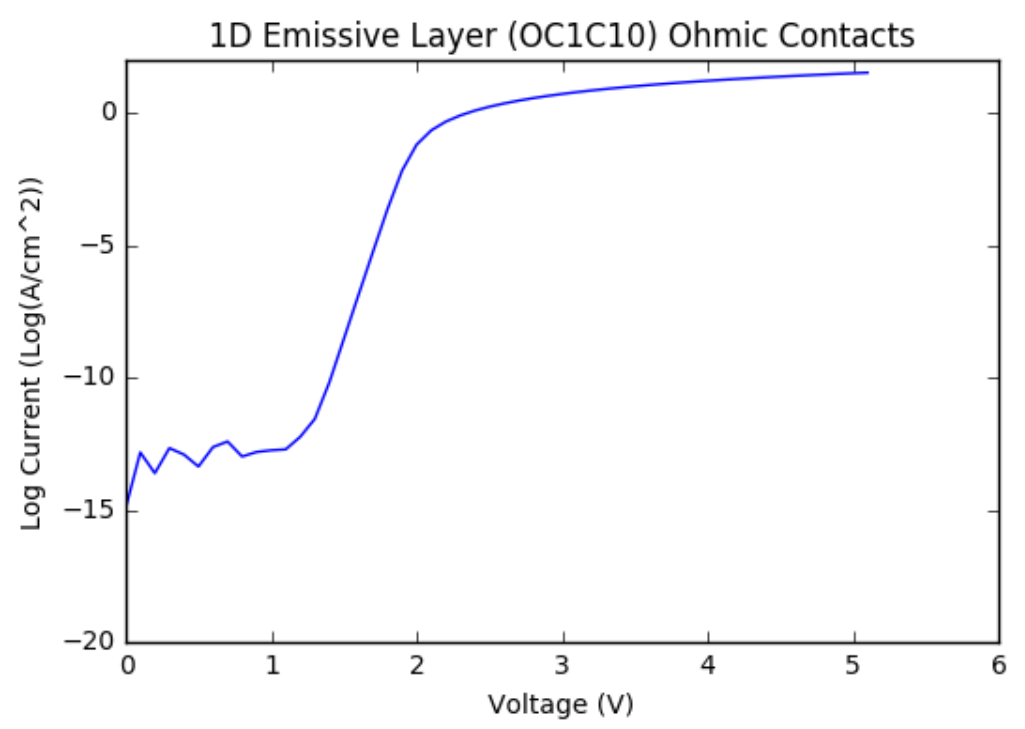

\section{Figure 5.8: Log I-V Plot of 1D Emissive Layer and Ohmic Contacts}

The log plot, Fig. 5.8, demonstrates the exponential growth of the current shown in the $1.5-2 V$ range with a slightly nonlinear growth at voltages above the turn-on voltage due to the Ohmic boundary conditions. The fluctuations observed below $1 \mathrm{~V}$ result from the insignificant magnitude of the Drift-Diffusion current in comparison to the Langevin recombination below the turn on voltage. Since the Drift-Diffusion current remains small, the Langevin recombination does not balance the smaller current densities from the continuity equations, so current conservation does not occur at the contacts until the excess carriers produce a large enough current that Langevin recombination balances the current continuity equations. Shockley Read Hall recombination due to the linear reliance on the carrier densities should balance the current

continuity equations at lower voltages if implemented. The following $\log \mathrm{I}-\mathrm{V}$ curves exhibit this same phenomenon. 


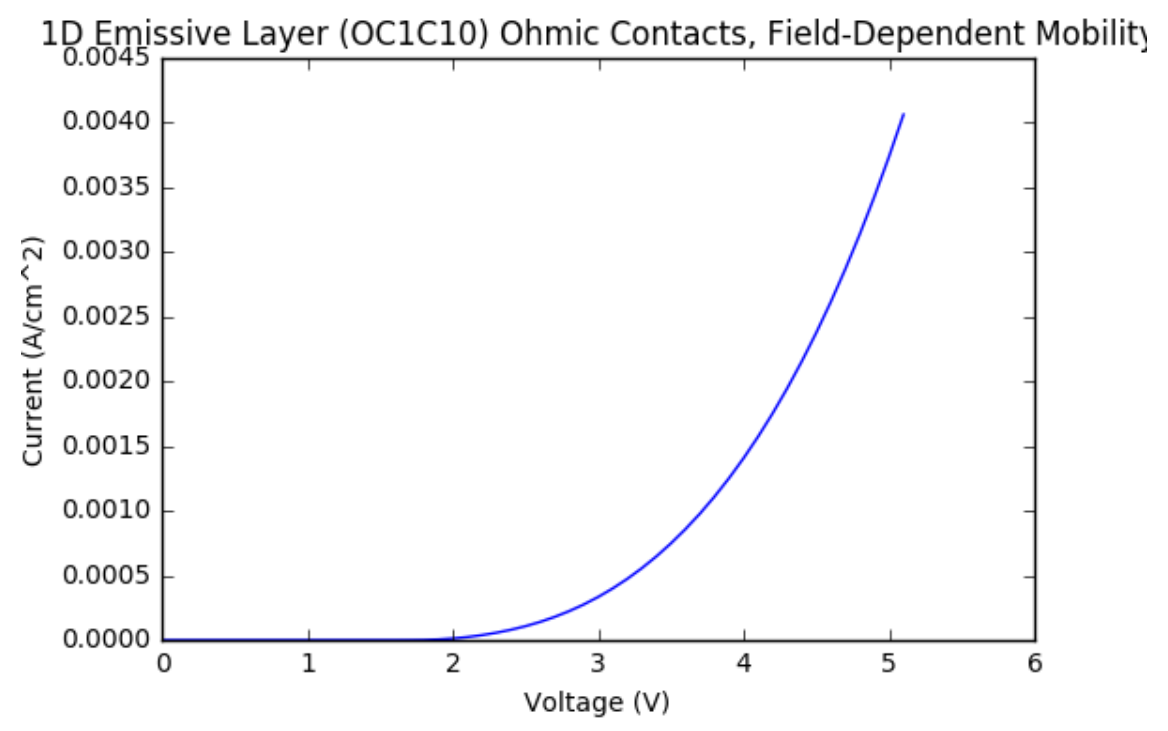

Figure 5.9: I-V Plot of 1D Emissive Layer, Ohmic Contacts, and FieldDependent Mobility

Fig. 5.9 also shows a nonlinear growth in current after the turn on voltage. However, the key difference comes in the growth of the current after the turn voltage. In Fig. 5.7, the current reaches $5 \frac{A}{c^{2}}$ with the application of $3 V$, however, the FieldDependent mobility decreases the overall current density yielding only $5 \frac{\mathrm{mA}}{\mathrm{cm}^{2}}$ with the application of $3 \mathrm{~V}$. The Field-Dependent mobility also affects the growth rate as the current reaches $240 \frac{\mathrm{mA}}{\mathrm{cm}^{2}}$ by $5 \mathrm{~V}$ whereas the constant mobility only reaches $30 \frac{\mathrm{A}}{\mathrm{cm} \mathrm{m}^{2}}$. 


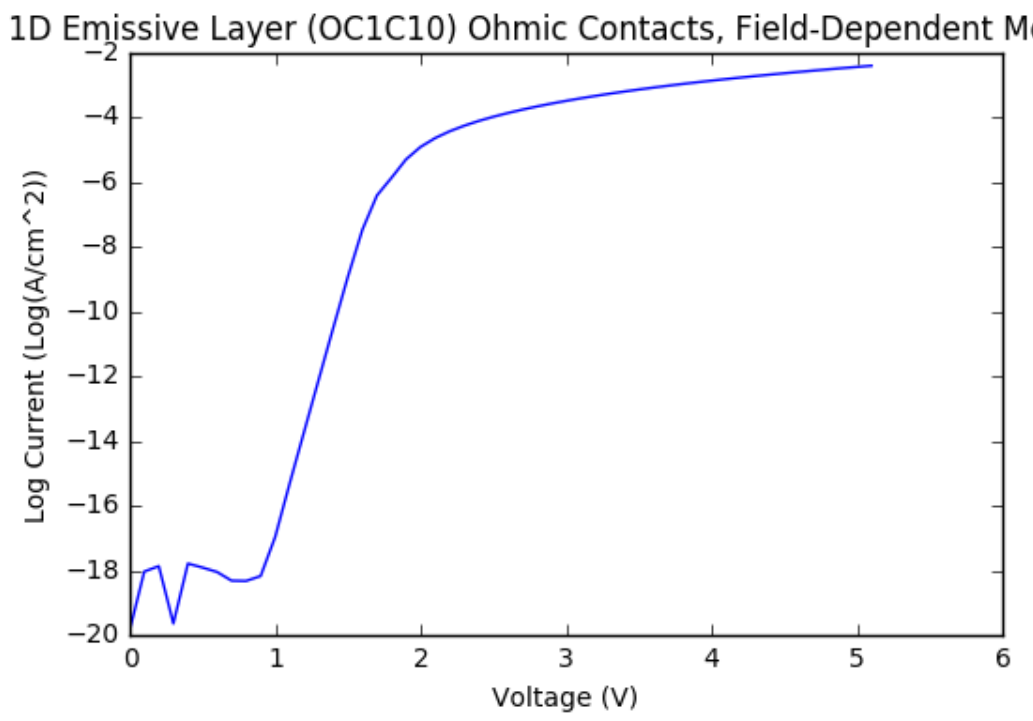

Figure 5.10: I-V Plot of 1D Emissive Layer, Ohmic Contacts, and FieldDependent Mobility

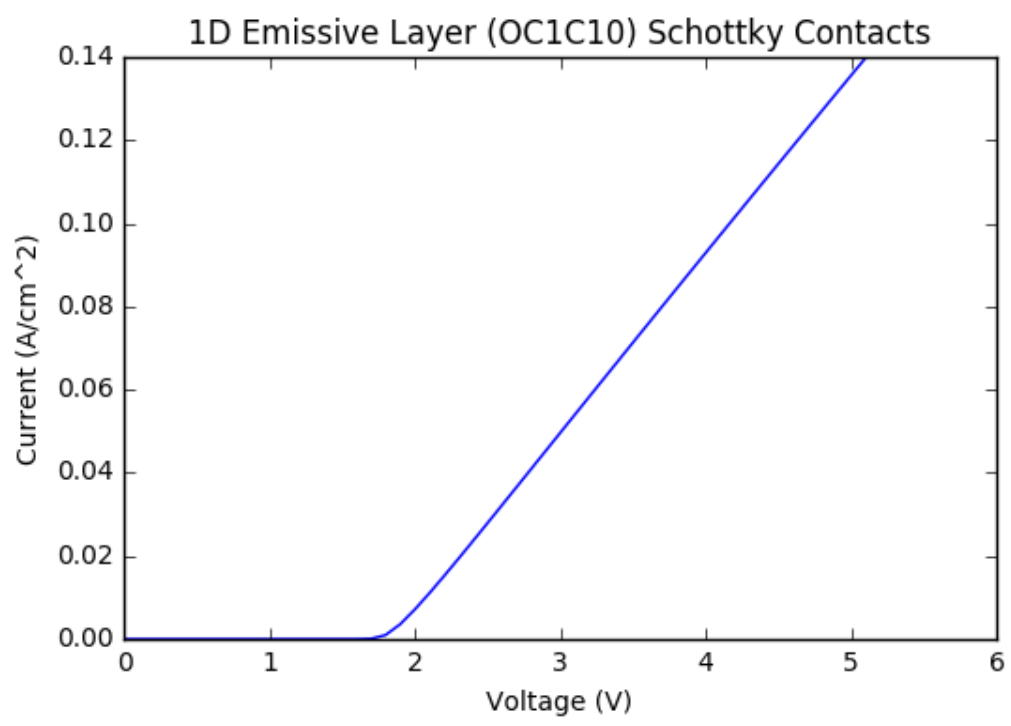

Figure 5.11: I-V Plot of 1D Emissive Layer and Schottky Contacts

Fig. 5.11 shows the first linear growth rate of the current as a function of voltage. This linear growth occurs due to the contact limiting Schottky current. The Schottky current, Sec. 3.2.6, shows a linear dependence on the excess carriers at the contact. The Schottky/Injection current instead of the Drift-Diffusion/Bulk current 
determines the overall current through the device. As a result, the magnitude of the current shows much lower values than those observed in Fig. 5.7. Furthermore, the current begins increasing below the bandgap at $1.9 \mathrm{eV}$ because of the smaller Fermi level separation caused by Schottky contacts. The difference between the workfunctions of the metals, Calcium and ITO, approximates to $1.9 \mathrm{eV}$ which coincides with the turn-on voltage.

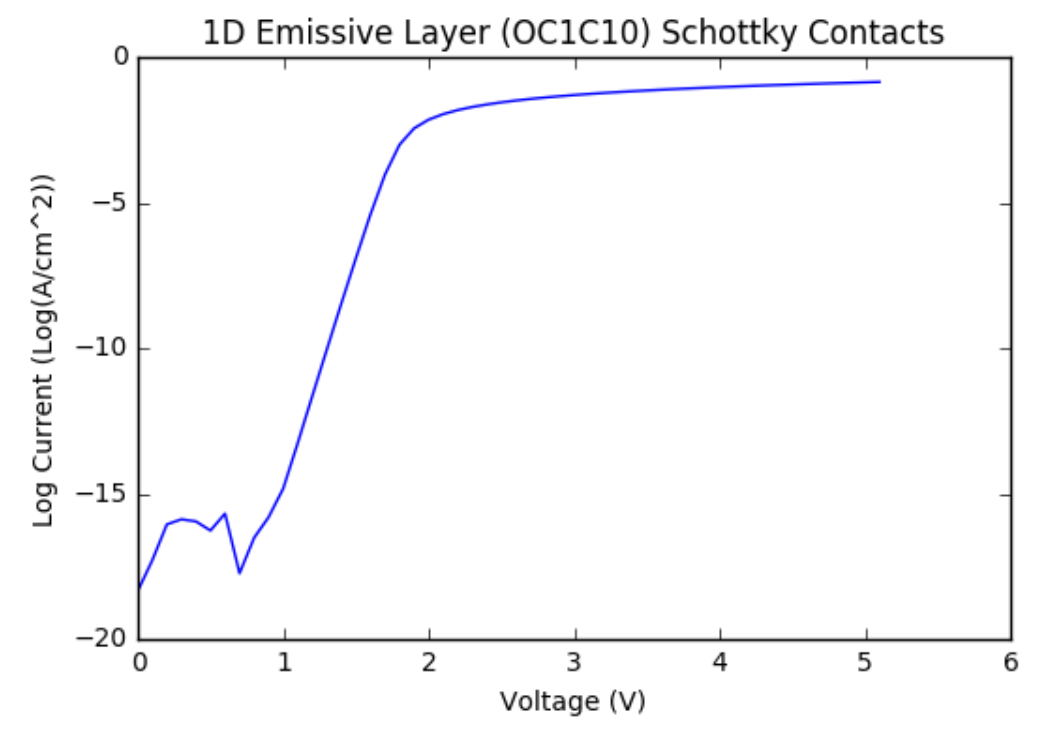

Figure 5.12: I-V Plot of 1D Emissive Layer and Schottky Contacts

Due to the linear growth seen in Fig. 5.11, Fig. 5.12 of this simulation instance shows a significant tapered growth after the turn on voltage in comparison to Figures 5.8 and 5.10. The ending point of the fluctuations due to the small carrier and current densities also shifts due to the smaller net bandgap of the metal workfunctions. Furthermore, Figures 5.12 and 5.14 show an unexpected, non-physical step increase in current density from $0-0.5 \mathrm{~V}$ in contrast to the Ohmic case which may result from high thermionic emission at low voltage.

To demonstrate the simulator's validity, the final LED simulation instance (Schottky Contacts, Field-Dependent Mobility) also contain the experimental results of a similar 
Organic LED. The EE 422 lab constructs an Organic LED utilizing the same materials as this simulation instance with the exception of an additional hole transport layer of PEDOT between the ITO and OC1C10 layers. The lab begins with the acetone bath, alcohol bath, and 15 minute UV Ozone baking of the ITO layered glass substrate. Then, PEDOT through spin-coating at 8000 RPM binds to the ITO on the substrate. After PEDOT application, $1 \mathrm{~mL}$ of a $7.5 \mathrm{~mL}$ solution of .5\% OC1C10-PPV and $99.5 \%$ Toluene deposits onto the PEDOT and again evenly distributes via spin-coating. Finally, the metal evaporation of Calcium occurs at $1.5 * 10^{-6}$ Torr producing a 4000 Angstrom cathode layer. This setup produces the experimental device shown in the results below.

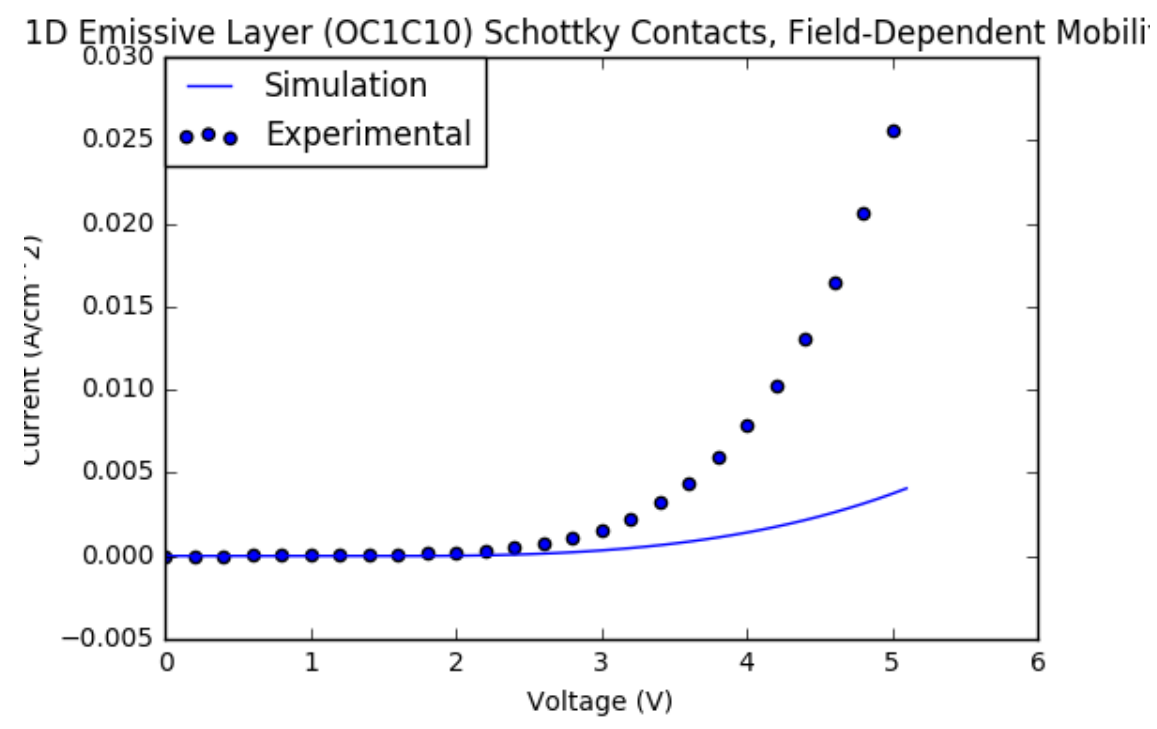

Figure 5.13: Simulated and Experimental I-V Plot of 1D Emissive Layer, Schottky Contacts, and Field-Dependent Mobility

Fig. 5.13 demonstrates well the Field-Dependent mobilities' effect on the I-V curve. Without the Field-Dependent mobility, Fig. 5.11, the current demonstrates a linear relationship with Schottky contacts, however, the decrease in current magnitude and nonlinear growth of the current represent the effects of the Field-Dependent mobility. The differences between Ohmic and Schottky contacts remain present as the turn-on 
voltage still shifts from $2.1 \mathrm{eV}$ to $1.9 \mathrm{eV}$ and the current drops by an order of magnitude shown in Figures 5.9 and 5.13.

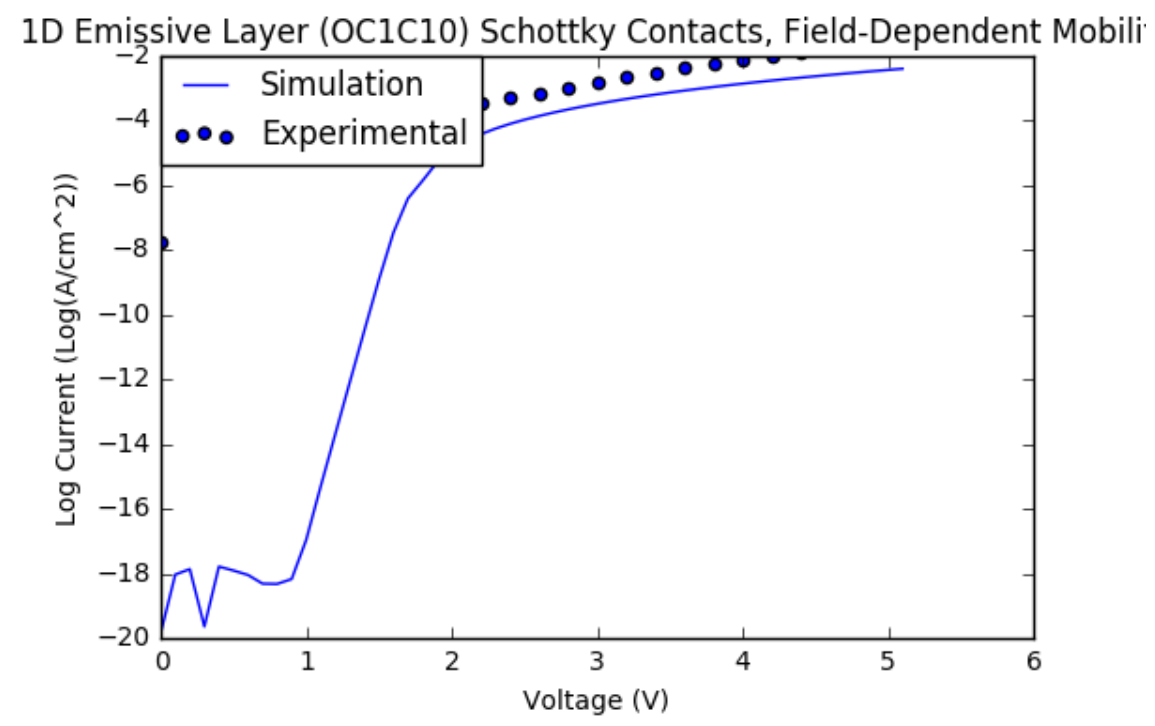

Figure 5.14: Simulated and Experimental I-V Plot of 1D Emissive Layer, Schottky Contacts, and Field-Dependent Mobility

Figures 5.13 and 5.14 demonstrate significant consistency with the experimental results. The absence of electron trapping in the simulation and the additional PEDOT layer in the experimental device may account for the discrepancy in the magnitude of the currents. 


\subsection{P-V Curves}

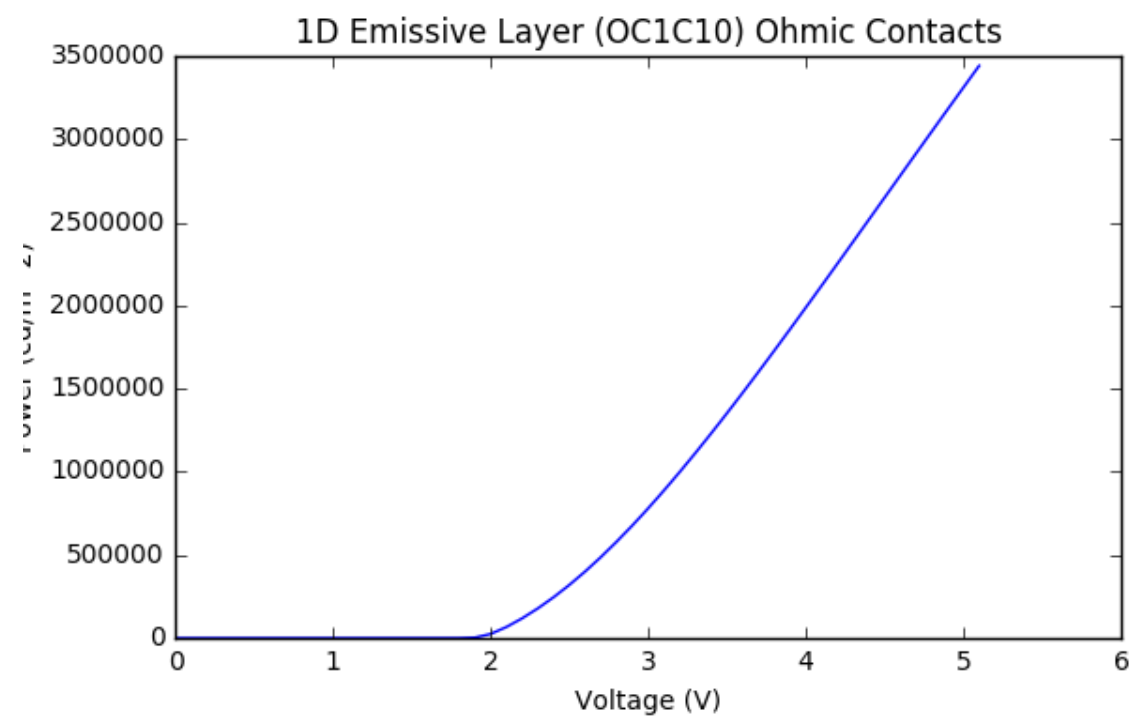

Figure 5.15: P-V Plot of 1D Emissive Layer and Ohmic Contacts

The P-V curve, Fig. 5.15, shows traditional LED operation with the turn on voltage occurring at the bandgap, $2.1 \mathrm{eV}$ for OC1C10-PPV. However, the large magnitude of the power output, $2 e 5-1.4 e 6 \frac{\mathrm{W}}{\mathrm{cm}^{2}}$, results from the large current densities, Fig. 5.7, and consequent Spontaneous Recombination. The position-dependent gain shows a net negative of $2200 \frac{1}{\mathrm{~cm}}$ demonstrating no contribution from Stimulated Emission. 


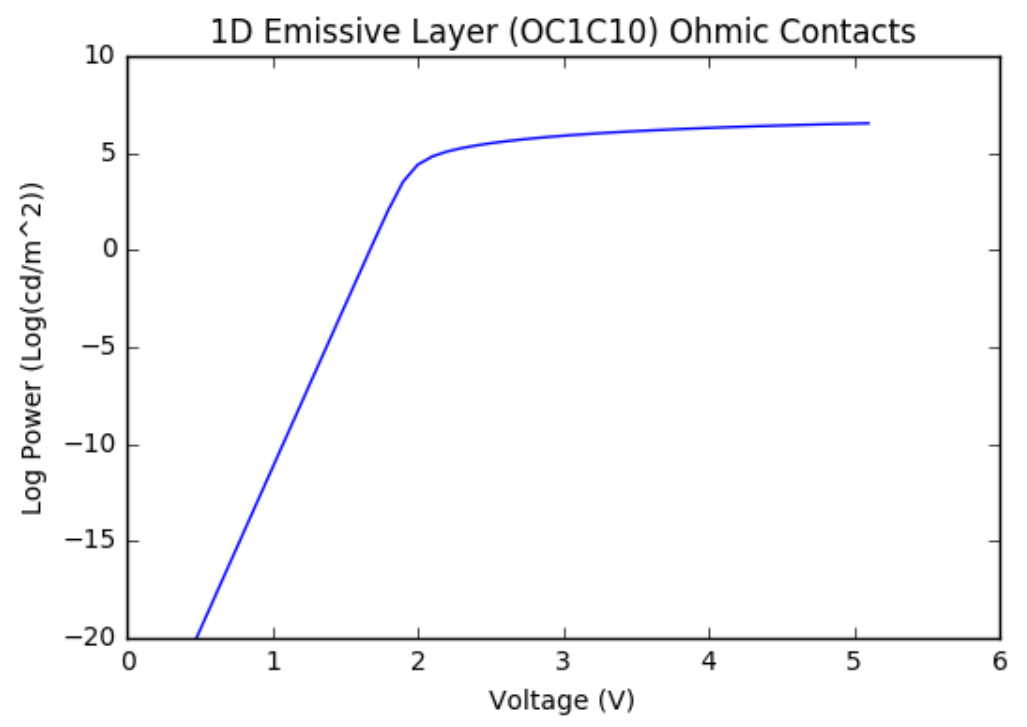

Figure 5.16: Semi-Log P-V Plot of 1D Emissive Layer and Ohmic Contacts

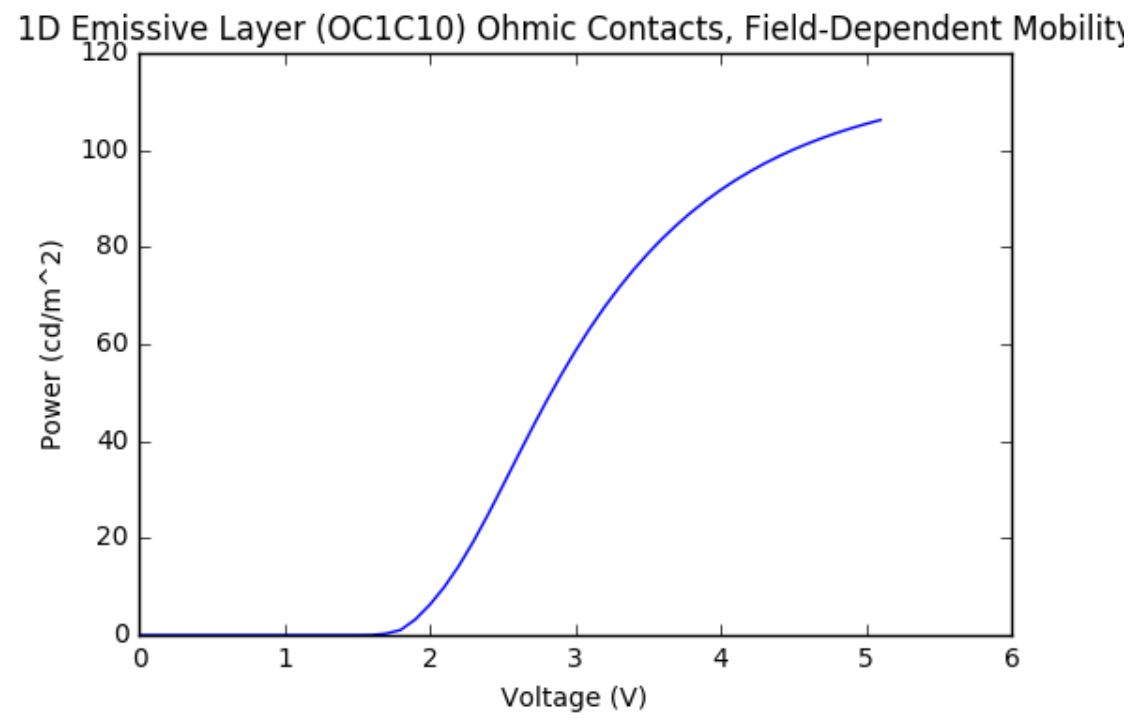

Figure 5.17: P-V Plot of 1D Emissive Layer and Ohmic Contacts and Field-Dependent Mobility

Fig. 5.17 demonstrates a significant drop in power output by three orders of magnitude due to including the Field-Dependent mobility. Furthermore, the growth rate shows significant bending in comparison to Fig. 5.15 resulting from the nonlinear dependence on the electric field. 


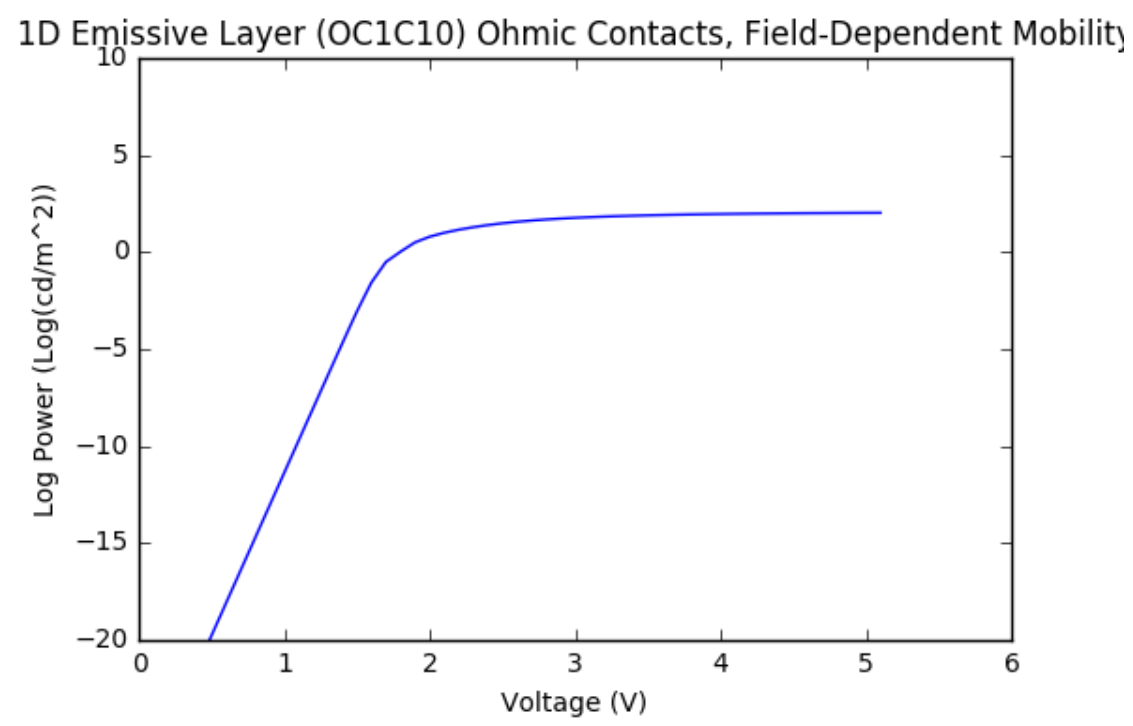

Figure 5.18: Semi-Log P-V Plot of 1D Emissive Layer and Ohmic Contacts

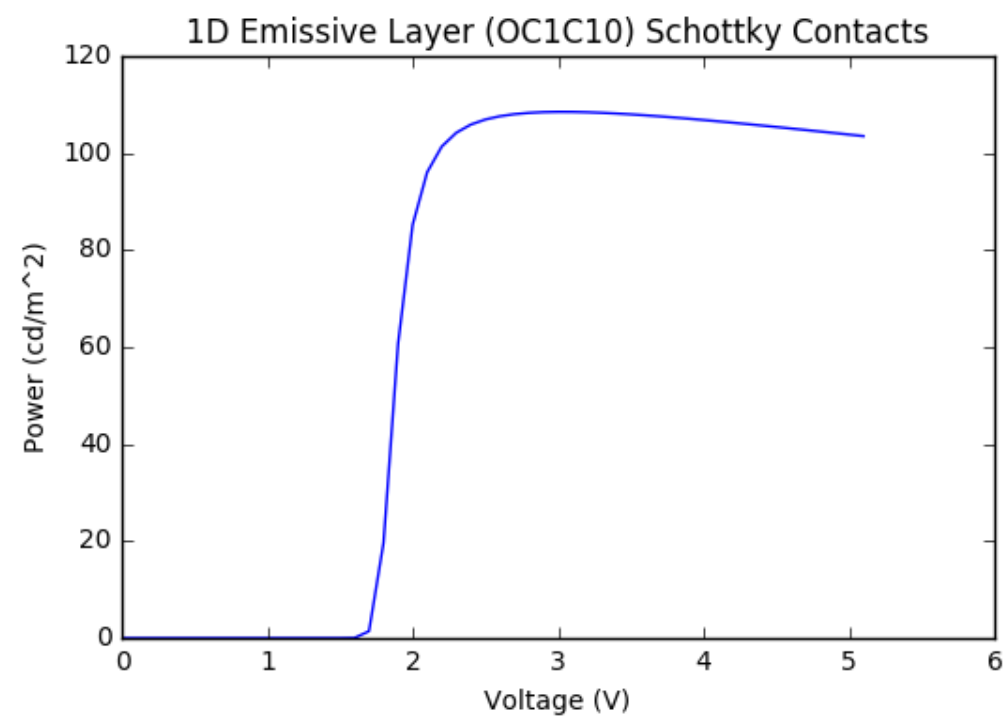

Figure 5.19: P-V Plot of 1D Emissive Layer and Schottky Contacts

Fig. 5.19 demonstrates the Schottky contacts' effect on the output power of the Organic LED. The turn on voltage shift effect as can be seen in the current corollary, Fig. 5.11, appears in the output power case as well. The turn on voltage shifts from $2.1 \mathrm{~V}$ to $1.9 \mathrm{~V}$ due to the smaller net bandgap between the contact workfunctions. The abrupt power saturation after the turn on voltage results from the contact current 
dominating the bulk current despite the quasi-Fermi level separating past the bandgap allowing for Spontaneous emission.

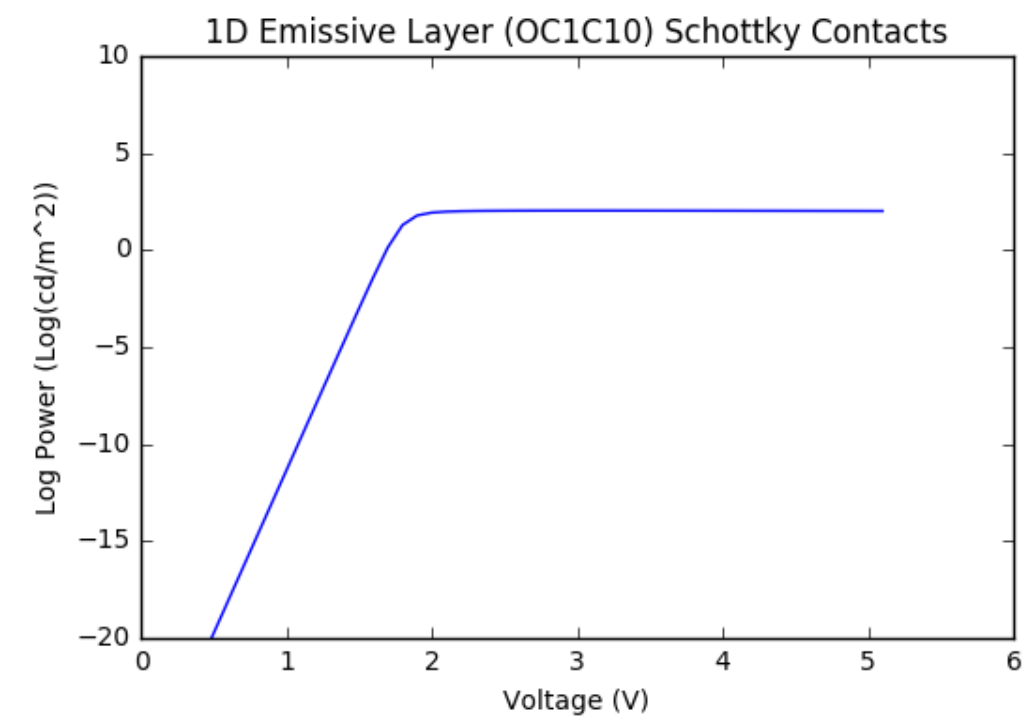

Figure 5.20: Semi-Log P-V Plot of 1D Emissive Layer and Schottky Contacts

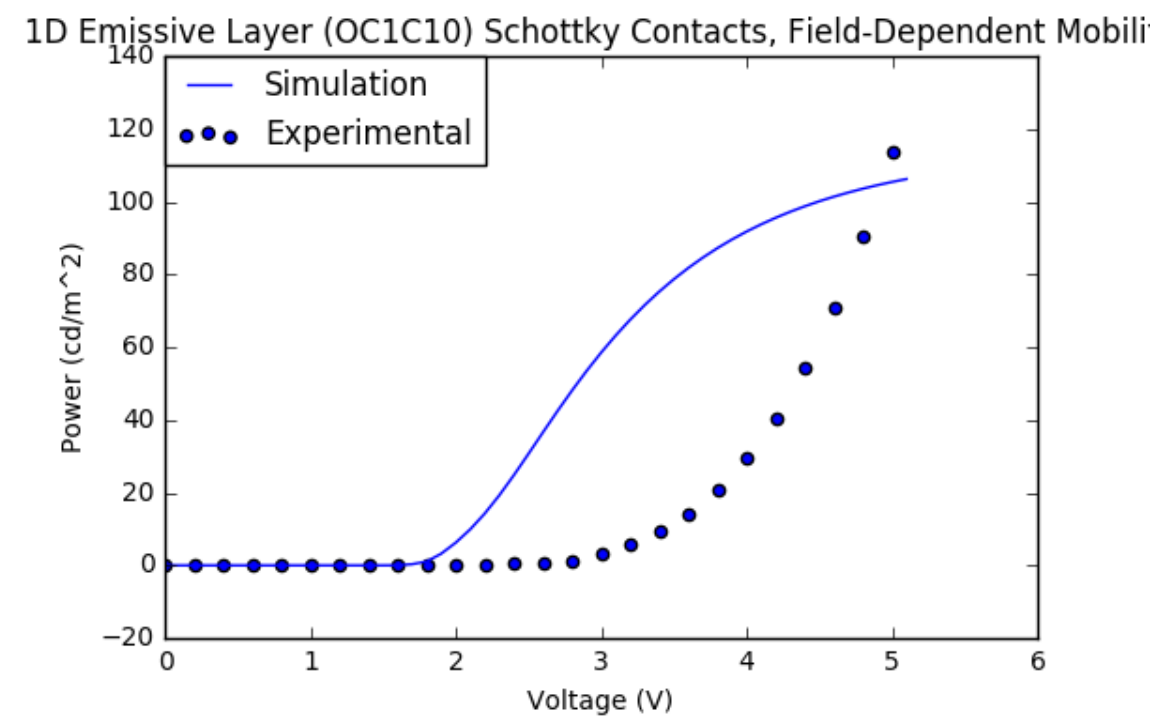

Figure 5.21: Simulated and Experimental P-V Plot of 1D Emissive Layer and Schottky Contacts 
Unlike Fig. 5.19, Fig. 5.21 demonstrates the nonlinear growth as seen in Fig. 5.17 despite the presence of the Schottky contacts. The Schottky contacts still limit the bulk current through device as seen by the lower power output, but the Field-Dependent mobility further lowers the current and hence the Spontaneous Emission.

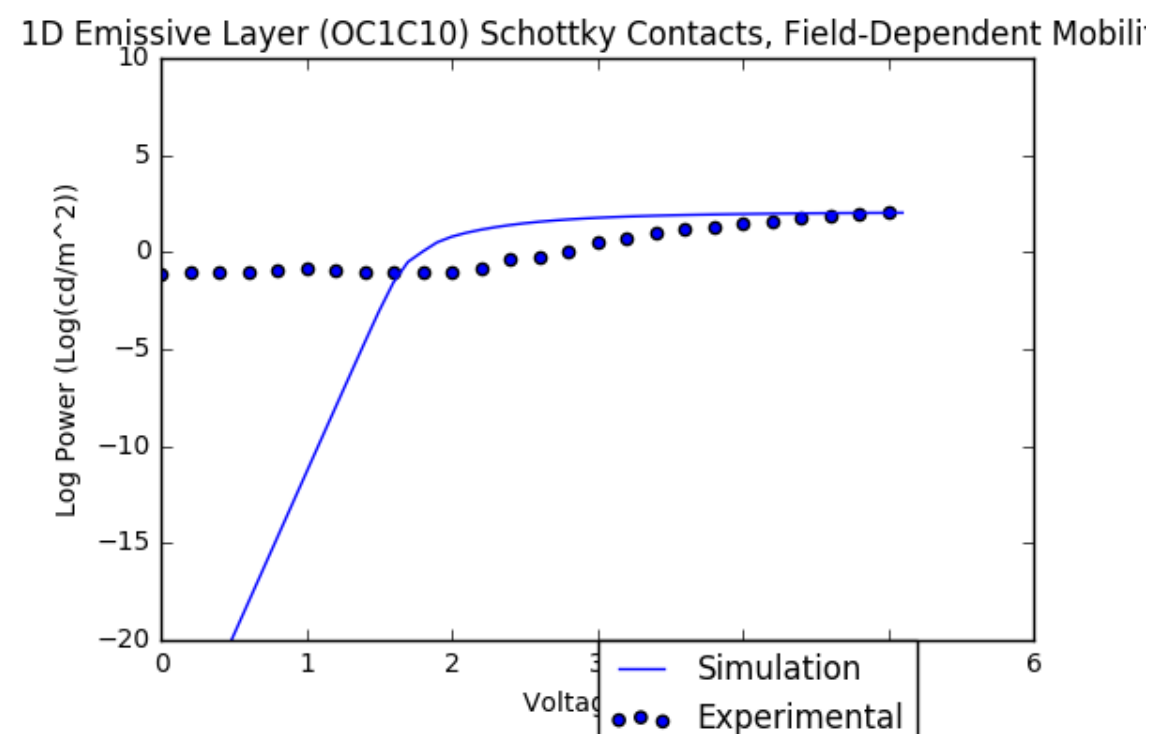

Figure 5.22: Simulated and Experimental Semi-Log P-V Plot of 1D Emissive Layer and Schottky Contacts

Figures 5.21 and 5.22 again show a fairly accurate replication of experimental results obtained from the EE 422 lab. The results displayed above demonstrate the simulator's capability of converging upon fairly accurate current and optical densities for a typical Organic LED as shown with the comparison to [19]. Lasing, however, requires carrier and optical confinement to yield population inversion. Sec. 6 focuses on attaining population inversion for positive optical gain. 
Chapter 6

\section{LASING SIMULATION}

Lasing utilizes carrier and optical confinement to produce population inversion. Population inversion allows for positive optical gain and stimulated emission. This project attempts lasing using the same LED structure as shown in Fig. 6.1 with higher contact reflectivity (98.9\%), higher applied voltage (0-25V), and increasing constant mobility shown in Table 6.1. For simplicity, this simulation did not use the FieldDependent mobility models. Tables 5.1, 5.2, 5.3 remain the same for this simulation, and tables 6.2 and 6.3 describe the material changes to the contacts. Appendix D contains the $\mathrm{I}-\mathrm{V}$ and $\mathrm{P}-\mathrm{V}$ plots obtained from the mobility and contact iterations.

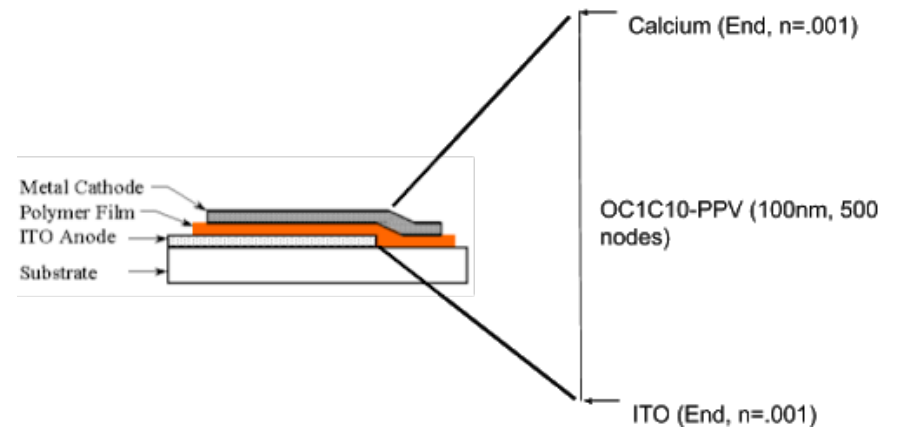

Figure 6.1: Laser Device Structure and Mesh Representation in Simulator

Table 6.1: Mobility Iterations

\begin{tabular}{|c|c|c|}
\hline Iteration & Electron Mobility $\frac{\mathrm{cm}^{2}}{V s}$ & Hole Mobility $\frac{\mathrm{cm}^{2}}{V s}$ \\
\hline Low Mobility & $5.1 \mathrm{e}-6$ & $5.1 \mathrm{e}-5$ \\
\hline Mid Mobility & $5.1 \mathrm{e}-3$ & $5.1 \mathrm{e}-2$ \\
\hline High Mobility & 5.1 & 51.0 \\
\hline
\end{tabular}


Table 6.2: Modified ITO Material Parameters

\begin{tabular}{|c|c|c|c|}
\hline Name & Value & Units & Description \\
\hline WorkFunction & -4.8 & $\mathrm{eV}$ & Work Function \\
\hline k_5e14 & 0.0023 & $\mathrm{~N} / \mathrm{A}$ & extinction coefficient at $5 \mathrm{e} 14 \mathrm{~Hz}$ \\
\hline n_5e14 & 0.001 & $\mathrm{~N} / \mathrm{A}$ & refractive index at $5 \mathrm{e} 14 \mathrm{~Hz}$ \\
\hline
\end{tabular}

Table 6.3: Modified Calcium Material Parameters

\begin{tabular}{|c|c|c|c|}
\hline Name & Value & Units & Description \\
\hline WorkFunction & -2.9 & $\mathrm{eV}$ & Calcium Work Function \\
\hline k_5e14 & 2.6362 & $\mathrm{~N} / \mathrm{A}$ & extinction coefficient \\
\hline n_5e14 & 0.001 & $\mathrm{~N} / \mathrm{A}$ & refractive index \\
\hline
\end{tabular}

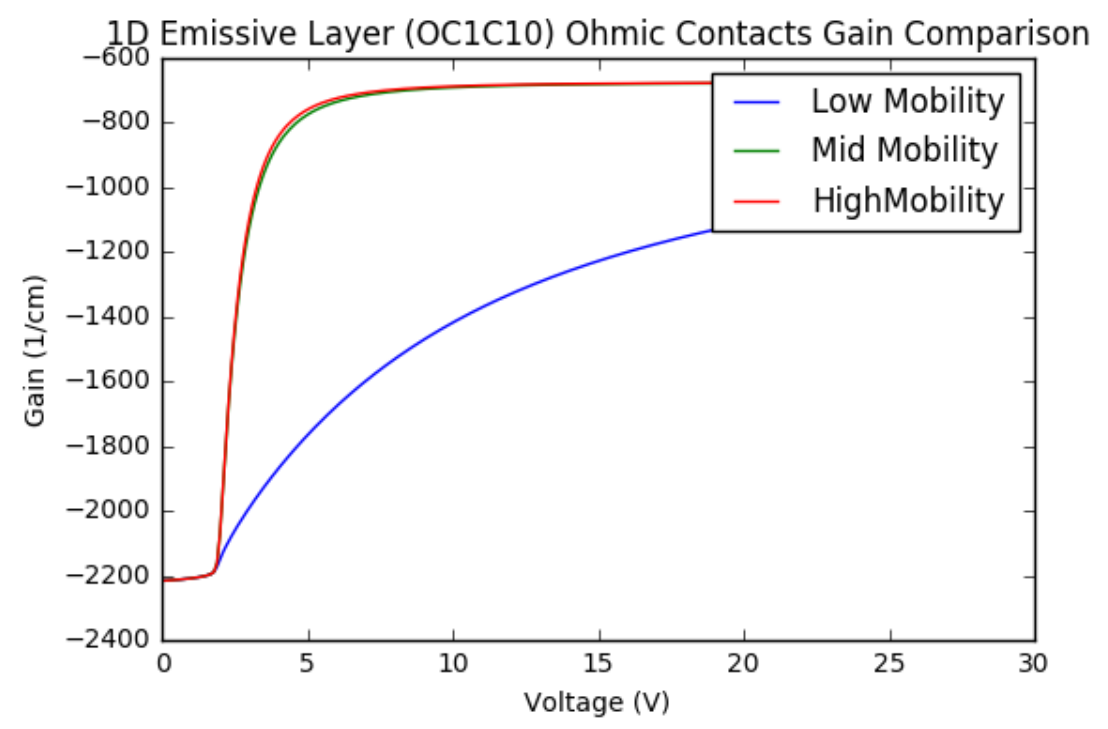

Figure 6.2: G-V Plot of 1D Emissive Layer with Ohmic Contacts

\subsection{Ohmic}

In Fig 6.2, 2.1V marks the starting point of increasing optical gain. This voltage coincides with the bandgap of OC1C10 as Stimulated Emission does not occur without a source, Spontaneous Emission. The Low Mobility represents the typical operating mobility in OC1C10-PPV which does not yield a system capable of lasing as population inversion can not be attained with these mobilities and device design. The higher 
mobilities reach and saturate at $-651 \frac{1}{\mathrm{~cm}}$. The gain and P-V curves saturate despite the continuing current increase as shown in the I-V curves in Appendix D. Figures 6.3 and 6.4 further show that the electron and hole quasi-Fermi levels saturate at the higher mobilities similar to gain. This saturation may occur due to carrier confinement failure as the current increases, but the quasi-Fermi levels remain constant at higher voltages and mobilities. Table 6.4 shows the color mapping of the different Fermi levels.

Table 6.4: Mobility Mapping

\begin{tabular}{|c|c|}
\hline Green & Low Mobility \\
\hline Yellow & Mid Mobility \\
\hline Orange & High Mobility \\
\hline Red & Band Energy \\
\hline
\end{tabular}

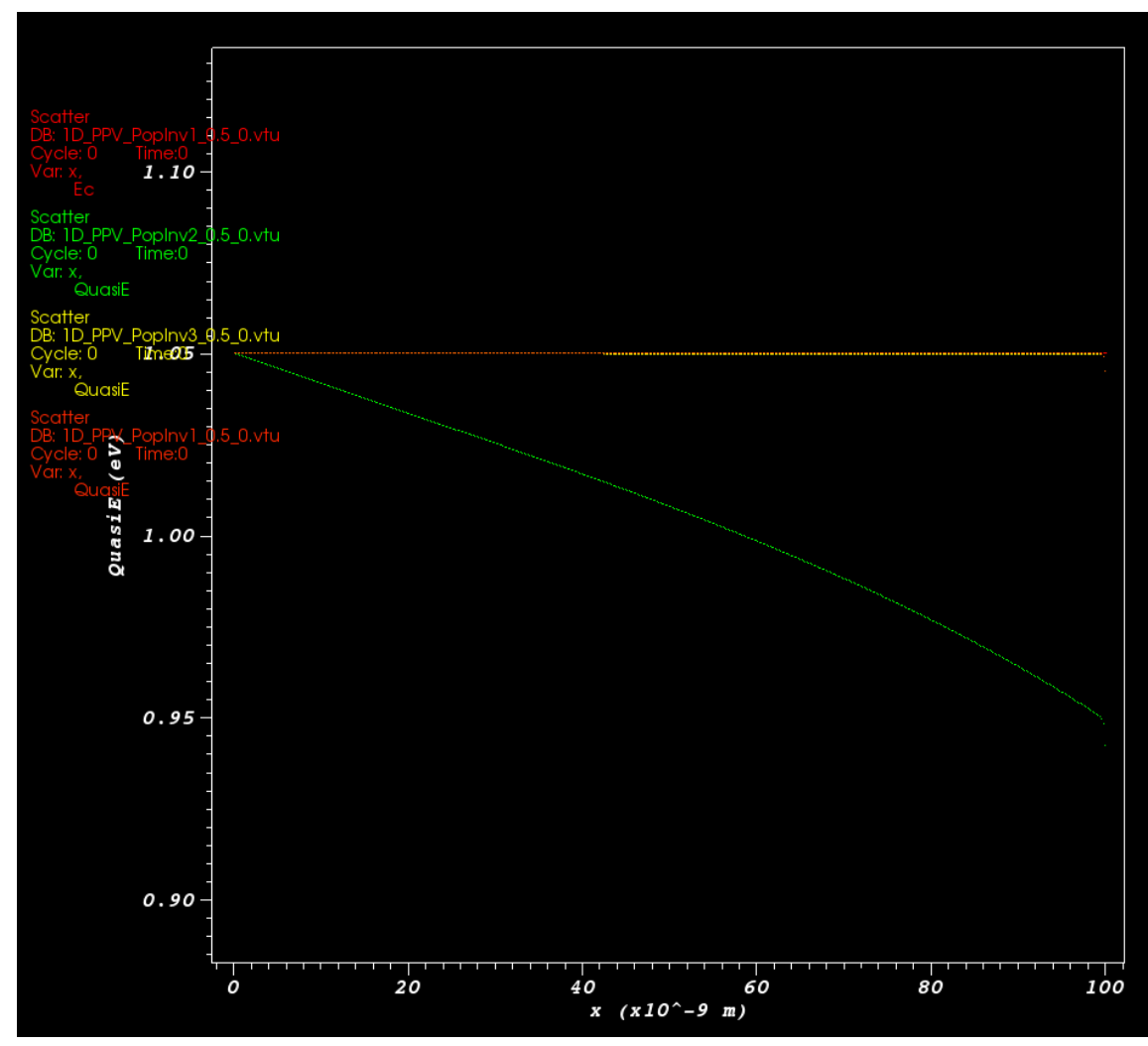

Figure 6.3: Electron Quasi Fermi Level at Low (Green), Mid (Yellow), and High (Orange) Mobilities at $25 \mathrm{~V}$ 


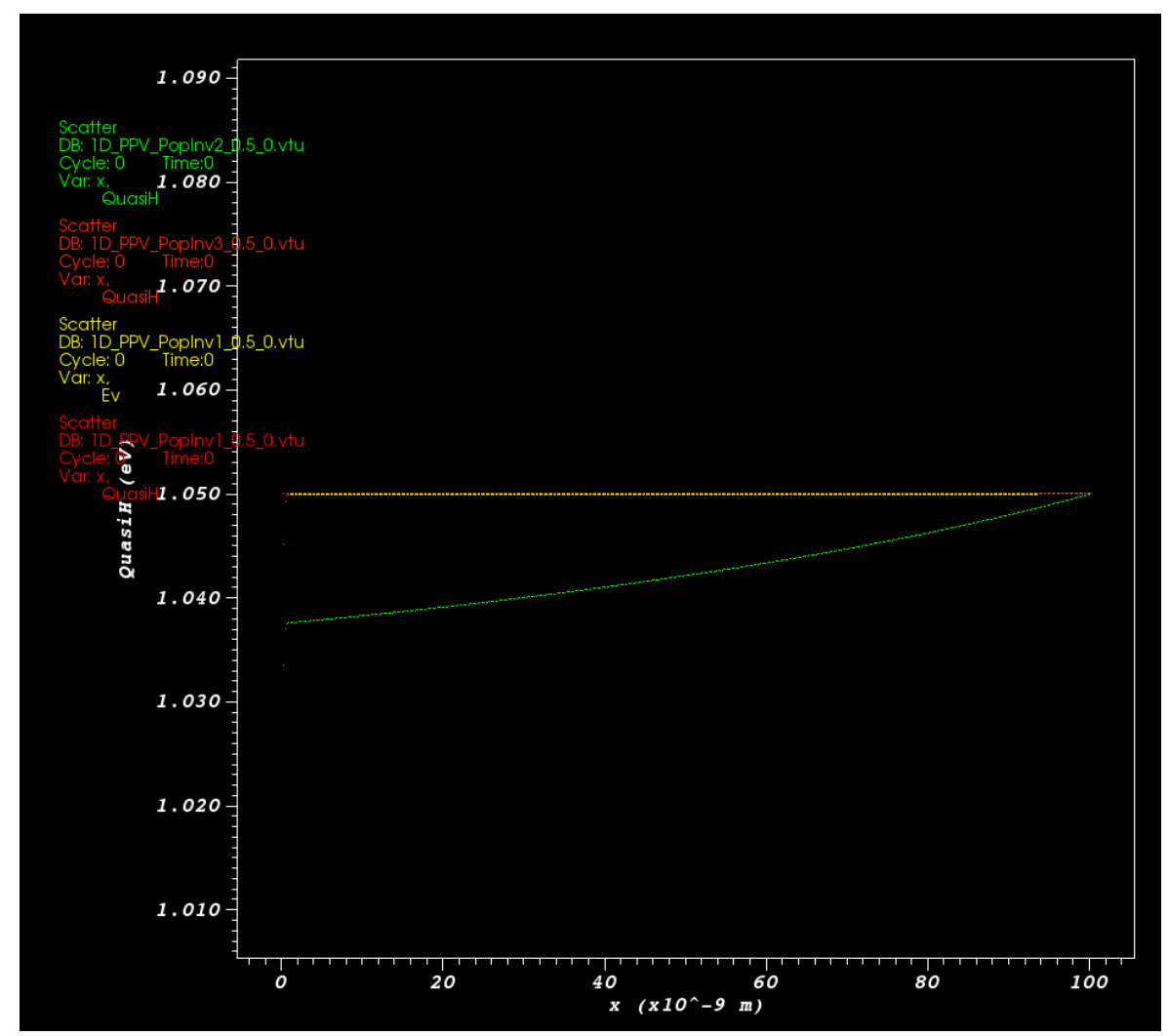

Figure 6.4: Hole Quasi Fermi Level at Low (Green), Mid (Yellow), and High (Orange) Mobilities at 25V 


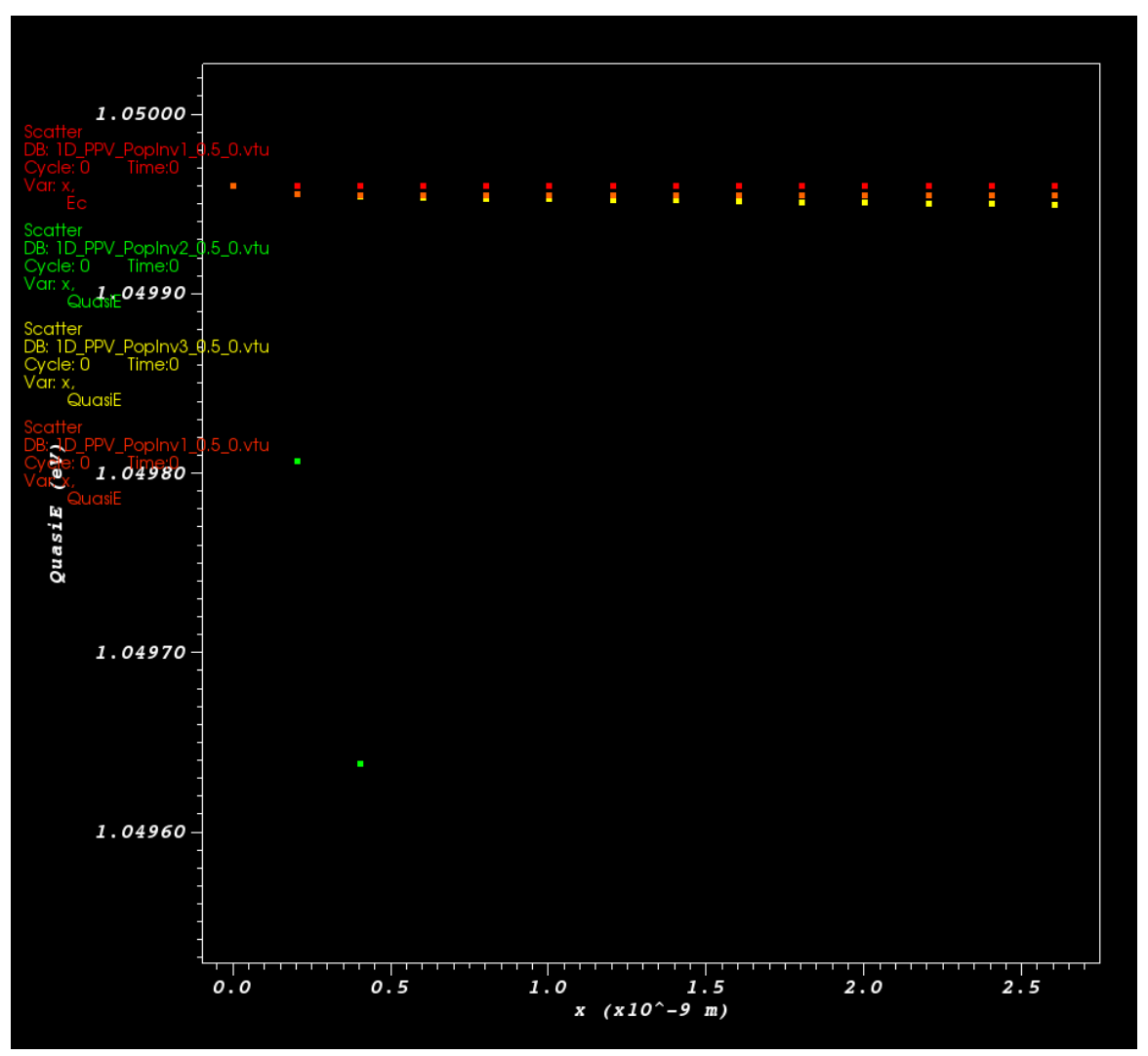

Figure 6.5: Zoomed Electron Quasi Fermi Level at Low (Green), Mid (Yellow), and High (Orange) Mobilities at 25V 


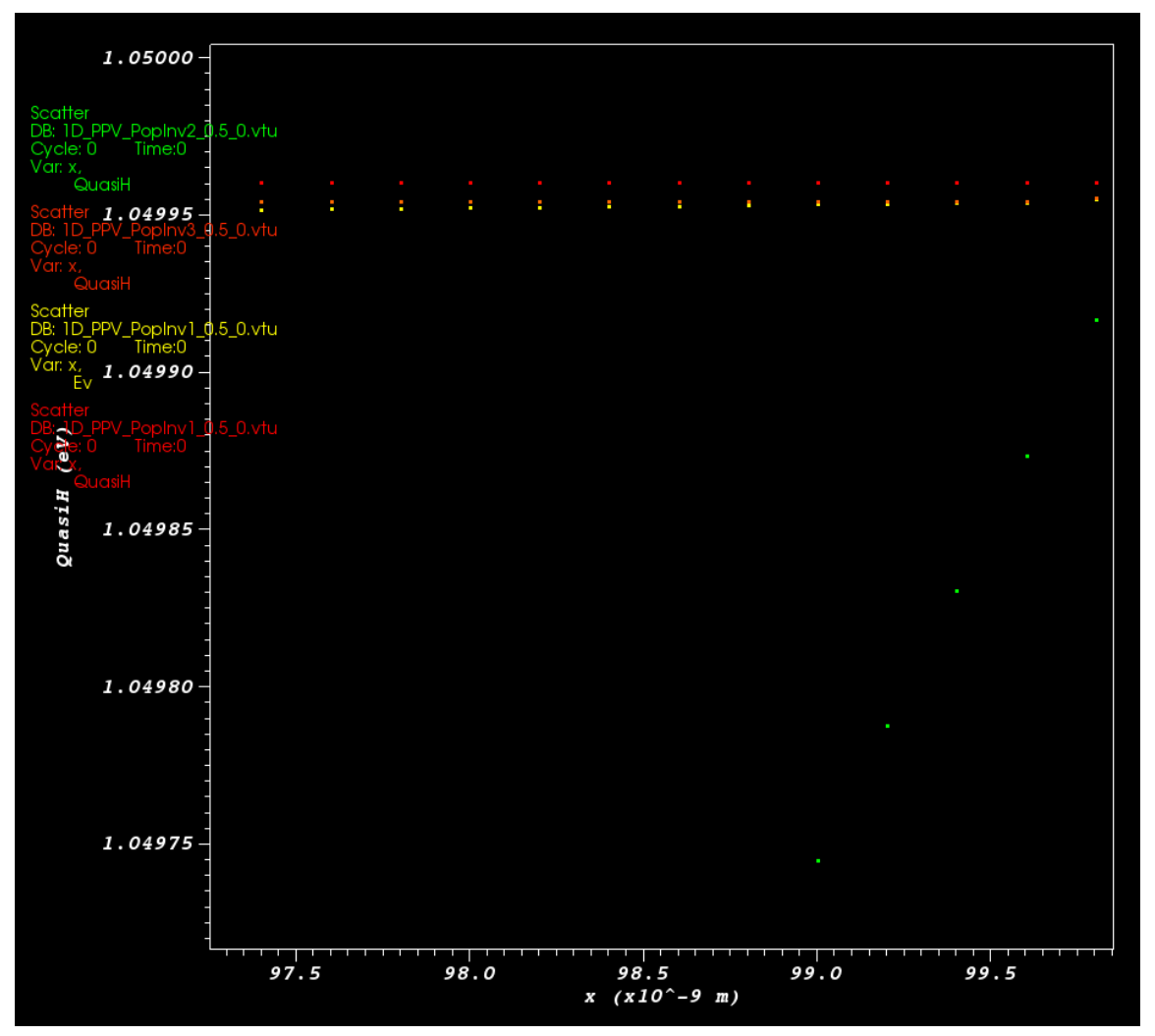

Figure 6.6: Zoomed Hole Quasi Fermi Level at Low (Green), Mid (Yellow), and High (Orange) Mobilities at $25 \mathrm{~V}$

Figures 6.5 and 6.6 show the minimal separation between Fermi levels and exemplify the saturation of carrier densities that occur at higher mobilities. Schottky contacts may provide better carrier confinement due to reduced current across the junction. 


\subsection{Schottky}

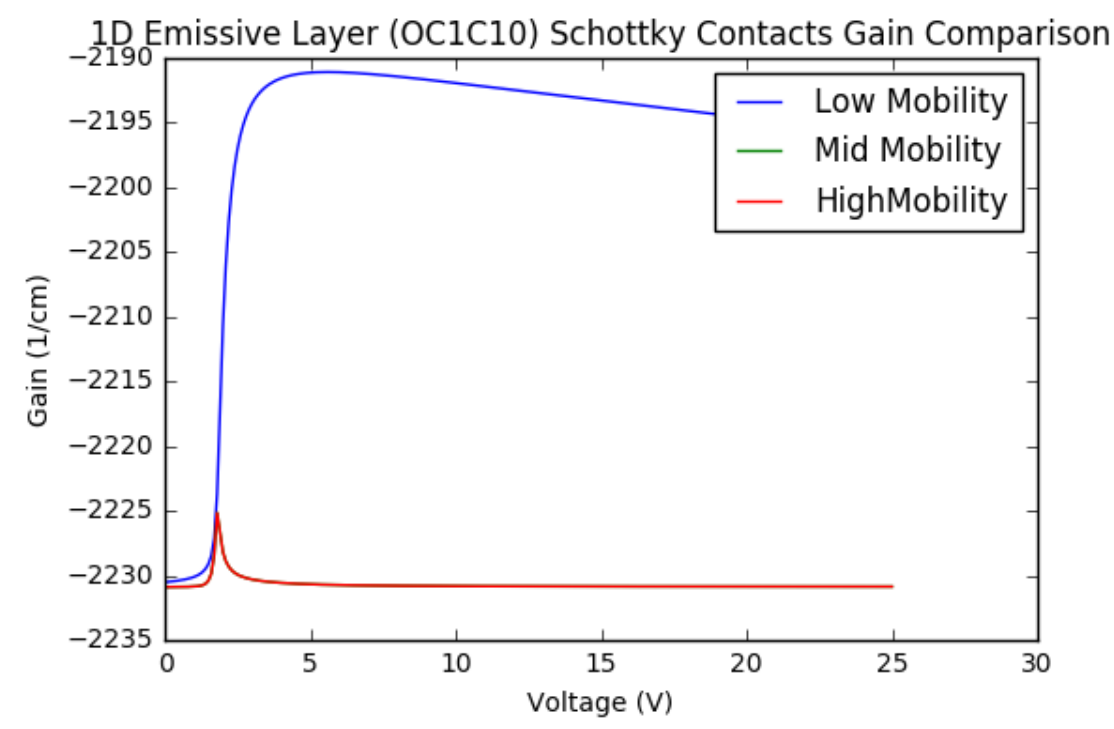

Figure 6.7: G-V Plot of 1D Emissive Layer with Schottky Contacts

Fig. 6.7 demonstrates the effect of current saturation from the Schottky current boundary condition. The carrier densities reach their saturation level and limit the overall current in the device through the Schottky boundary condition. The Schottky contacts in decreasing the allowable current at high voltages decreases the optical gain because the Schottky current instead of Spontaneous Recombination maintains current continuity. 


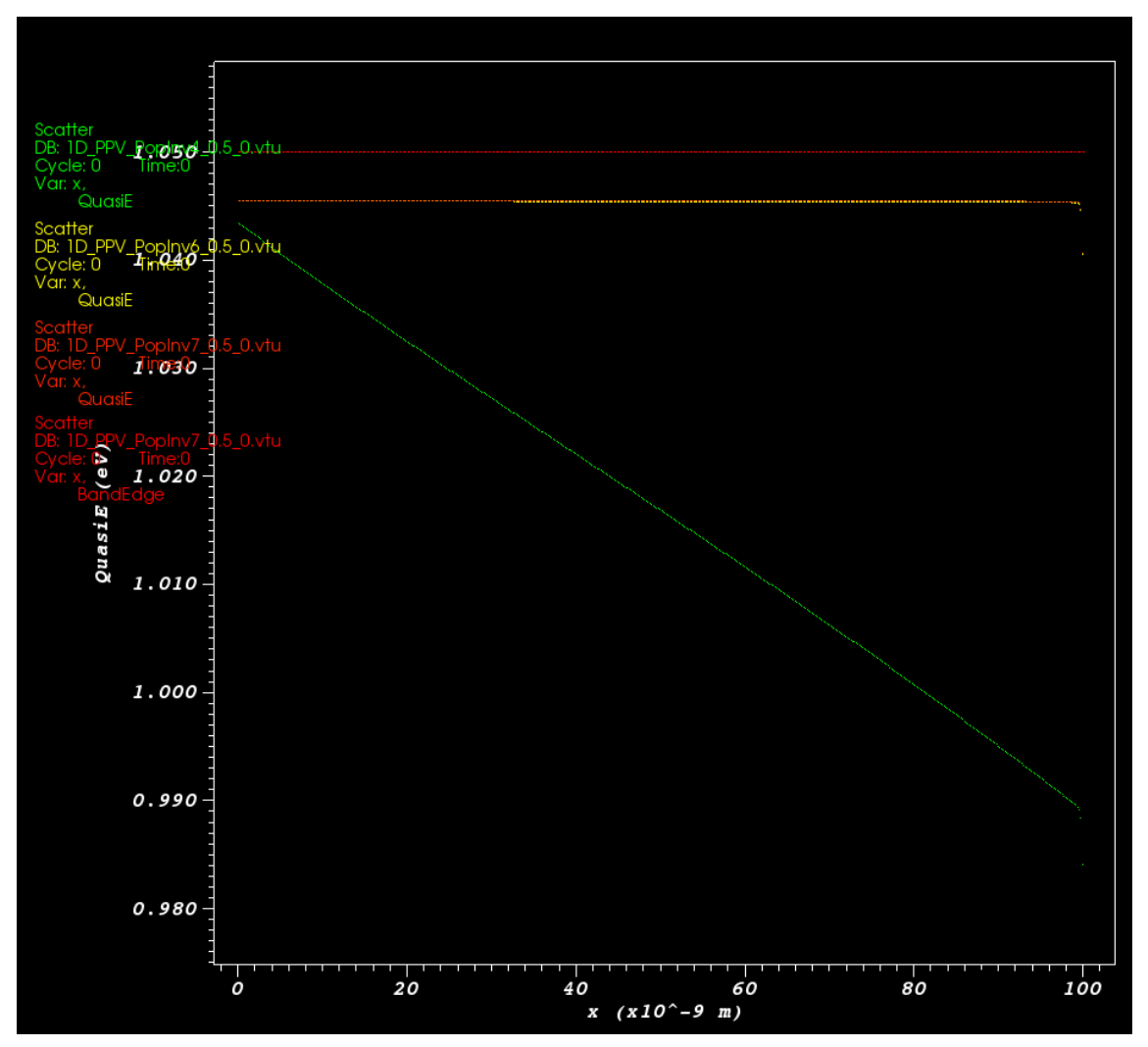

Figure 6.8: Electron Quasi Fermi Level at Low (Green), Mid (Yellow), and High (Orange) Mobilities at 25V 


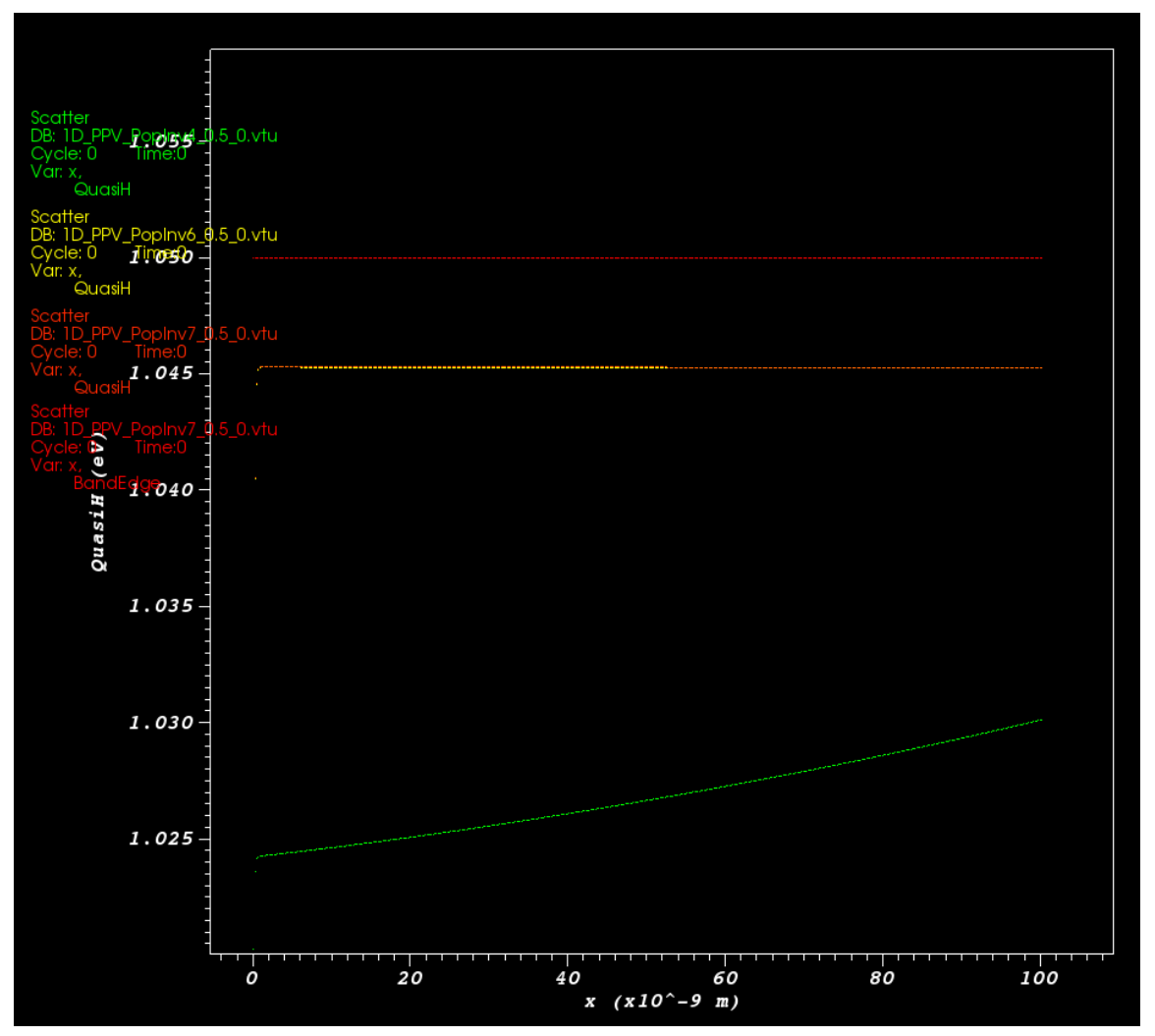

Figure 6.9: Hole Quasi Fermi Level at Low (Green), Mid (Yellow), and High (Orange) Mobilities at 25V 


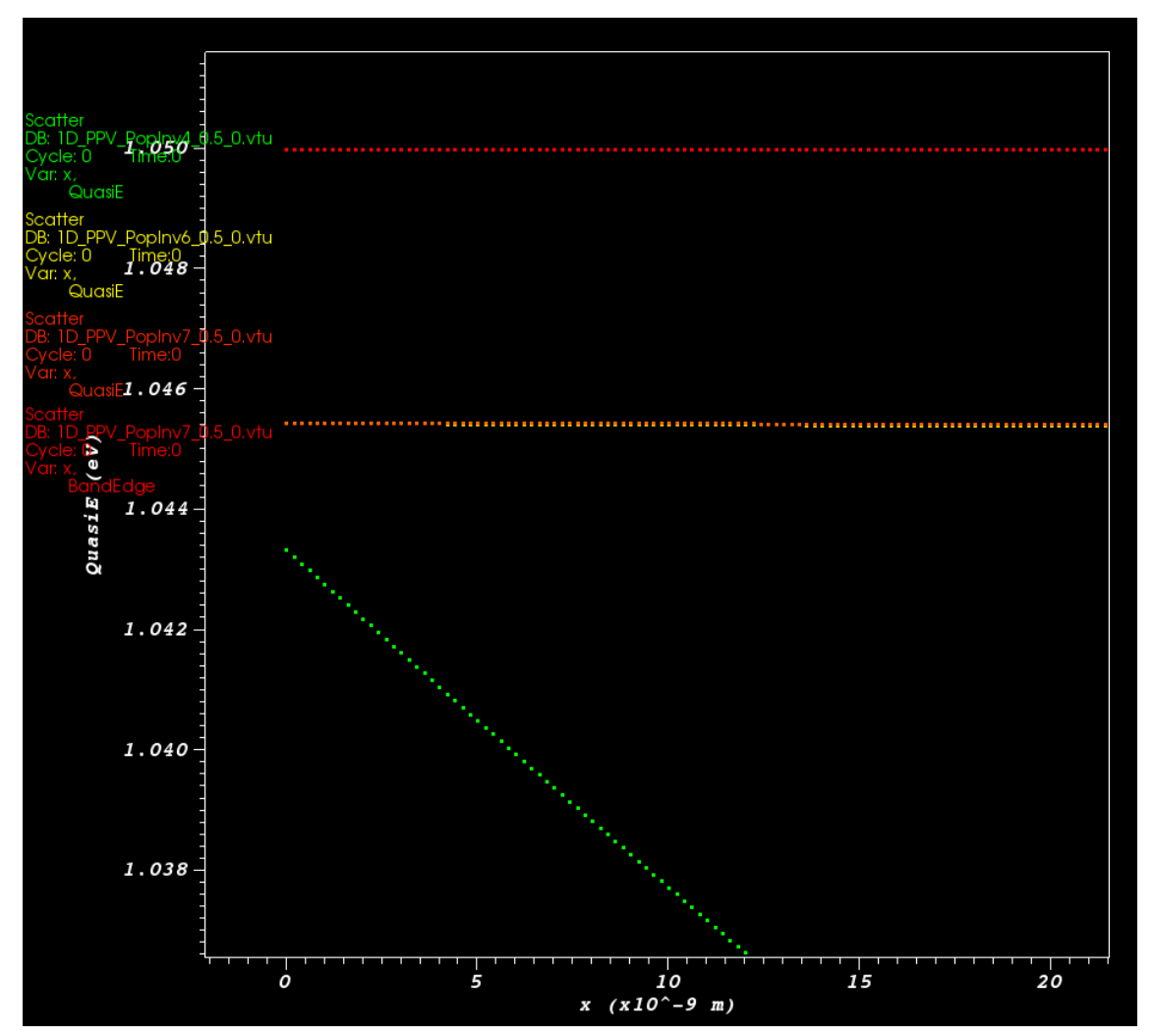

Figure 6.10: Zoomed Electron Quasi Fermi Level at Low (Green), Mid (Yellow), and High (Orange) Mobilities at 25V 


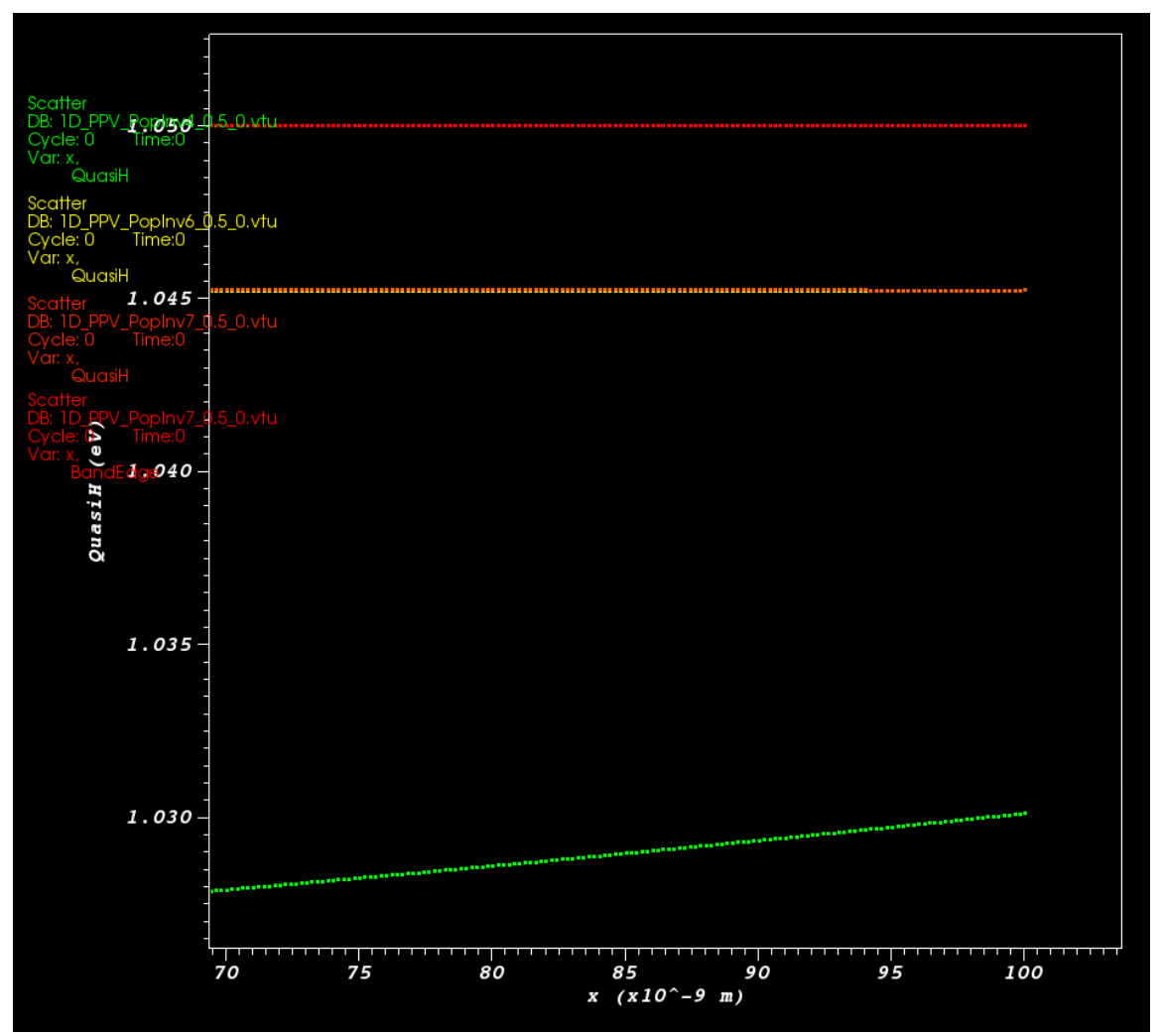

Figure 6.11: Zoomed Hole Quasi Fermi Level at Low (Green), Mid (Yellow), and High (Orange) Mobilities at $25 \mathrm{~V}$

The decreased carrier saturation reflects in figures 6.8 and 6.9 where the quasi-Fermi levels saturated at fewer electron Volts, 1.045 instead of 1.049. The zoomed figures 6.10 and 6.11 show a more linear decrease in the quasi-Fermi levels in comparison to the exponential decrease in the Ohmic counterparts which may be due to the linear current at the contacts. The decrease in gain suggests that the desired device structure needs to be capable of high injection and high containment. The introduction of blocking layers should improve carrier confinement. These blocking layers should have larger gaps in their mobilities to limit carrier escape from the emissive layer. Lasing did not occur in this instance, but the addition of blocking layers to confine the carriers and exciton rate equations may provide an initial estimate to begin physical synthesis. 
Chapter 7

\section{CONCLUSION AND FUTURE WORK}

The simulator produces the electrostatic potential, electron, hole, transverse optical field, and photon densities per node in the device region. These solutions then update dependent models such as total current, gain, recombination, and optical power output. Table 7.1 shows the complete set of implemented models available in the simulator. With the correct combination of implemented models and material parameters, the simulator accurately reproduces theoretical and experimental results as seen in ??. This simulator provides the basis to begin constructing structures to test for positive optical gain in electrically-pumped organic devices. 


\section{Table 7.1: Implemented Equations and Constituent Models}

\begin{tabular}{|l|}
\hline Equations: Models \\
\hline Poisson: \\
- E-Field \\
- D-Field \\
- $\rho($ Free,Trapped, Ion $)$ \\
\hline Current Continuity: \\
- Drift-Diffusion \\
\hline Recombination: \\
- Shockley-Read-Hall \\
- Auger \\
- Langevin \\
- Stimlated \\
\hline Helmholtz: \\
- Effective Index \\
- Optical Intensity \\
\hline Photon Rate: \\
- Photon Generation \\
- Photon Absorption \\
- Optical Power Output \\
\hline Boundary Conditions: \\
- Ohmic \\
- Schottky \\
- Perfect Electric Conductor \\
\hline
\end{tabular}

Furthermore, this simulator relies on an open-source Finite Volume Solver, DEVSIM, and open-source 1-D Helmholtz solver allowing for availability and customization to future users. The simulator also contains a material database that allows for simple maintenance of available materials. With this framework, future work should focus on finding a device structure with the currently implemented models to confine the the carriers for lasing. Once a device structure yields a positive optical gain, the next step should confirm that positive optical gain still occurs with charge carrier quenching produced by the Exciton Rate equation and at a temperature below the melting point of the polymer given by Energy Balance Transport equation as shown in 7.1 [6] and $7.2[8]$. Physical implementation can begin once the set of material parameters and device structure in the simulator yield a lazing diode with these additional models. 


$$
\frac{\partial S_{i}}{\partial \mathrm{t}}=G_{i} R(n, p)+\vec{\nabla} \cdot \vec{J}_{i}-\left(k_{i}^{(r)}+k_{i}^{(n)}\right) S_{i}-k_{i}^{(a)} f S_{i}^{2}+\sum_{j=S, T}\left(k_{j} S_{j}-k_{i j} S_{i}\right)-k_{\mathrm{TPQ}}(n+p) .
$$

Figure 7.1: Exciton Rate Equation Including Singlet/Triplet Excitons and Charge Carrier Quenching [6]

For electrons the Energy Balance Transport Model consists of:

$$
\begin{aligned}
& \operatorname{div} \vec{S}_{n}=\frac{1}{q} \vec{J}_{n} \cdot \vec{E}-W_{n}-\frac{3 k}{2} \frac{\partial}{\partial t}\left(\lambda_{n}{ }^{*} T_{n}\right) \\
& \vec{J}_{n}=q D_{n} \nabla n-q \mu_{n} n \nabla \psi+q n D_{n}^{T} \nabla T_{n} \\
& \vec{S}_{n}=-K_{n} \vec{\nabla} T_{n}-\left(\frac{k \delta_{n}}{q}\right) \vec{J}_{n} T_{n}
\end{aligned}
$$

and for holes:

$$
\begin{aligned}
& \operatorname{div} \vec{S}_{p}=\frac{1}{q} \vec{J}_{p} \cdot \vec{E}-W_{p}-\frac{3 k}{2} \frac{\partial}{\partial t}\left(\lambda_{p}^{*} p T_{p}\right) \\
& \vec{J}_{p}=-q D_{p} \nabla p-q \mu_{p} p \nabla \psi-q p D_{p}^{T} \nabla T_{p} \\
& \vec{S}_{p}=-K_{p} \nabla T_{p}-\left(\frac{k \delta_{p}}{q}\right) \vec{J}_{p} T_{p}
\end{aligned}
$$

Figure 7.2: Energy Balance Transport Model yielding Heat Transport [8] 
Bibliography

[1] Nir Tessler. "Lasers Based on Semiconducting Organic Materials". In: Advanced Materials 11.5 (1999), pp. 363-370. DOI: 10.1002/(SICI) 1521-4095(199903) $11: 5<363:$ : AID-ADMA363>3 . 0 . CO ; 2-Y. eprint: https : / / onlinelibrary . wiley.com/doi/pdf/10.1002/ \%28SICI \\%291521-4095\\%28199903\\%2911】 $\% 3 \mathrm{~A} 5 \backslash \% 3 \mathrm{C} 363 \backslash \% 3 \mathrm{~A} \backslash \% 3 \mathrm{AAID}-\mathrm{ADMA} 363 \backslash \% 3 \mathrm{E} 3.0 . \mathrm{CO} \backslash \% 3 \mathrm{~B} 2-\mathrm{Y}$. URL: https : //onlinelibrary . wiley . com/doi/abs/10 . 1002/\%5C\%28SICI\%5C\%291521$4095 \% 5 \mathrm{C} \% 28199903 \% 5 \mathrm{C} \% 2911 \% 5 \mathrm{C} \% 3 \mathrm{~A} 5 \% 5 \mathrm{C} \% 3 \mathrm{C} 363 \% 5 \mathrm{C} \% 3 \mathrm{~A} \% 5 \mathrm{C} \% 3 \mathrm{AAID}-\mathrm{ADMA} 363 \%$ $5 \mathrm{C} \% 3 \mathrm{E} 3.0 . \mathrm{CO} \% 5 \mathrm{C} \% 3 \mathrm{~B} 2-\mathrm{Y}$.

[2] David Hoagland. "The physics of polymers: Concepts for understanding their structures and behavior, by Gert R. Strobl, Springer-Verlag, New York, 1996. ISBN 3-540-60768-4". In: Journal of Polymer Science Part A: Polymer Chemistry 35.7 (1997), pp. 1337-1338. DOI: 10 . 1002 / (SICI ) 1099-0518(199705) 35 : 7<1337 : : AID-POLA20>3 . 0 . CO ; 2-8. eprint: https : / / onlinelibrary . wiley . com/doi/pdf/10 . 1002/ \%28SICI \\%291099-0518\\%28199705\\%2935\ $\% 3 \mathrm{~A} 7 \backslash \% 3 \mathrm{C} 1337 \backslash \% 3 \mathrm{~A} \backslash \% 3 \mathrm{AAID}-\mathrm{POLA} 20 \backslash \% 3 \mathrm{E} 3.0$. CO \\%3B2 - 8. URL: https : //onlinelibrary . wiley . com/doi/abs/10 . 1002/\%5C\%28SICI\%5C\%2910990518\%5C $\% 28199705 \% 5 C \% 2935 \% 5 C \% 3 A 7 \% 5 C \% 3 C 1337 \% 5 C \% 3 A \% 5 C \% 3 A A I D-P O L A 20 \%$ $5 \mathrm{C} \% 3 \mathrm{E} 3.0 . \mathrm{CO} \% 5 \mathrm{C} \% 3 \mathrm{~B} 2-8$.

[3] G. Hadziioannou and G.G. Malliaras. Semiconducting Polymers: Chemistry, Physics and Engineering. Wiley, 2006. ISBN: 9783527312719. URL: https : // books . google. com/books? id=2mq8NAEACAAJ.

[4] David Braun. "Semiconducting polymer LEDs". In: Materials Today 5.6 (2002), pp. 32-39. ISSN: 1369-7021. DOI: https://doi.org/10.1016/S1369-7021(02) 
00637-5. URL: http : //wWw . sciencedirect.com/science/article/pii / S1369702102006375.

[5] P. Bhattacharya. Semiconductor Optoelectronic Devices. Prentice Hall, 1997. ISBN: 9780134956565. URL: https://books.google.com/books?id=wWaiSgAACAAJ.

[6] J. P. Dakin and Robert G. W. Brown. Handbook of Optoelectronics (Two-Volume Set). Bristol, PA, USA: Taylor \& Francis, Inc., 2006. ISBN: 0750306467.

[7] Bjorn Sjodin. What's The Difference Between FEM, FDM, and FVM? Apr. 2016. URL: https : / / www . machinedesign . com/fea-and-simulation/what-sdifference-between-fem-fdm-and-fvm.

[8] Silvaco. URL: https://www.silvaco.com/.

[9] Silvaco. Atlas User's Manual. Sept. 2012. URL: https://dynamic.silvaco.com/ dynamicweb/jsp/downloads/DownloadManualsAction.do?req=silentmanuals\& $\mathrm{nm}=\mathrm{atlas}$

[10] Multiphysics simulation just got better, faster, more accessible. URL: https : / / WWW. comsol. com/.

[11] DEVSIM Manual. URL: https://devsim.net/.

[12] Hank Childs et al. "VisIt: An End-User Tool For Visualizing and Analyzing Very Large Data". In: High Performance Visualization-Enabling Extreme-Scale Scientific Insight. Oct. 2012, pp. 357-372.

[13] Robert Entner. "Modeling and simulation of negative bias temperature instability". In: ().

[14] J. Van Zeghbroeck. Principles of Semiconductor Devices. Bart Van Zeghbroeck, 2011. URL: https://books .google. com/books?id=hw3YtwEACAAJ. 
[15] W. L. Engl, H. K. Dirks, and B. Meinerzhagen. "Device modeling". In: Proceedings of the IEEE 71.1 (Jan. 1983), pp. 10-33. ISSN: 0018-9219. DOI: 10.1109/PROC. 1983.12524 .

[16] V. Palankovski and R. Quay. Analysis and Simulation of Heterostructure Devices. Computational Microelectronics. Springer Vienna, 2012. ISBN: 9783709105603. URL: https: //books . google. com/books?id=9p\%5C_zCAAAQBAJ.

[17] P Schwaha et al. "Advanced Equation Processing for TCAD". In: (Jan. 2006).

[18] Jinn-Liang Liu. "Scharfetter-Gummel Method". In: (Dec. 2016), p. 2.

[19] Paul Blom and Michel Vissenberg. "Charge transport in poly(p-phenylene vinylene) light-emitting diodes". In: Materials Science and Engineering: R: Reports 27 (May 2000), pp. 53-94. DOI: 10.1016/S0927-796X(00)00009-7.

[20] David Braun. "Electronic injection and conduction processes for polymer devices". In: Journal of Polymer Science Part B: Polymer Physics 41.21 (2003), pp. 26222629. DOI: 10 . 1002/polb . 10654. eprint: https : //onlinelibrary . wiley . com/doi/pdf/10.1002/polb.10654. URL: https://onlinelibrary.wiley . com/doi/abs/10.1002/polb.10654.

[21] E.H. Rhoderick and R.H. Williams. Metal-semiconductor contacts. Monographs in electrical and electronic engineering. Clarendon Press, 1988. ISBN: 9780198593362. URL: https://books . google. com/books?id=0zcoAQAAMAAJ.

[22] L.A. Coldren, S.W. Corzine, and M.L. Mashanovitch. Diode Lasers and Photonic Integrated Circuits. Wiley Series in Microwave and Optical Engineering. Wiley, 2012. ISBN: 9781118148181. URL: https : / / books . google $\cdot$ com / books ? id= GBB1kOYONT4C.

[23] T. Numai. Fundamentals of Semiconductor Lasers. Springer Series in Optical Sciences. Springer Japan, 2014. ISBN: 9784431551485. URL: https ://books . google. com/books?id=UbpeBAAAQBAJ. 
[24] Z. Q. Li and Z. M. S. Li. "Comprehensive Modeling of Superluminescent LightEmitting Diodes". In: IEEE Journal of Quantum Electronics 46.4 (Apr. 2010), pp. 454-461. ISSN: 0018-9197. DOI: 10.1109/JQE.2009.2032426.

[25] C. Henry. "Theory of spontaneous emission noise in open resonators and its application to lasers and optical amplifiers". In: Journal of Lightwave Technology 4.3 (Mar. 1986), pp. 288-297. ISSN: 0733-8724. DOI: 10.1109/JLT.1986.1074715.

[26] Frederic Nataf. "Absorbing boundary conditions and perfectly matched layers in wave propagation problems". In: Radon Series on Computational and Applied Mathematics 11 (Jan. 2013). DOI: 10.1515/9783110282283.219.

[27] R. Fitzpatrick. Classical Electromagnetism. CreateSpace Independent Publishing Platform, 2016. ISBN: 9781530325917. URL: https : // books . google.com / books?id=pSi2jwEACAAJ.

[28] D. Marcuse. "Classical derivation of the laser rate equation". In: IEEE Journal of Quantum Electronics 19.8 (Aug. 1983), pp. 1228-1231. ISSN: 0018-9197. DOI: 10.1109/JQE. 1983.1072025.

[29] Ifor Samuel and Graham Turnbull. "Polymer lasers: Recent advances". In: Materials Today 7 (Sept. 2004), 28fffdfffdffd35. DOI: 10.1016/S1369-7021(04) 00399-2.

[30] Harald Hoppe, N S. Sariciftci, and D Meissner. "Optical constants of conjugated polymer/fullerene based bulk-heterojunction organic solar cells". In: Molecular Crystals and Liquid Crystals - MOL CRYST LIQUID CRYST 385 (Jan. 2002), pp. 113-119. DOI: 10.1080/713738799.

[31] Mikhail N. Polyanskiy. Refractive index database. URL: https://refractiveindex. info (visited on 04/30/2019). 
[32] Yau Wong and Guangrui Li. "Exact finite difference schemes for solving Helmholtz equation At any wavenumber". In: International Journal of Numerical Analysis and Modeling. Series B 1 (Jan. 2011).

[33] Cal Poly Github. http://www.github.com/CalPoly.

[34] Formatting Guidelines. http://grad.calpoly.edu/files/2017-2018\%20Masters\% 20Thesis\%20Formatting\%20Guidelines.pdf.

[35] K. Horio and H. Yanai. "Numerical modeling of heterojunctions including the thermionic emission mechanism at the heterojunction interface". In: IEEE Transactions on Electron Devices 37.4 (Apr. 1990), pp. 1093-1098. ISSN: 0018-9383. DOI: $10.1109 / 16.52447$

[36] E. Glytsis. Slab Dielectric Waveguides. Dec. 2018. URL: http://users.ntua.gr/ eglytsis/IO/Slab_Waveguides_Chapter.pdf. 


\title{
APPENDICES
}

\author{
Appendix A
}

USEFUL LINKS

- Simulator Github Repository: https://github.com/Bob95132/EE599-Thesis

- DEVSIM Github Repository: https://github.com/devsim/devsim

- DEVSIM Manual: https://devsim.net/ 


\title{
Appendix B
}

\section{SCHARFETTER-GUMMEL DERIVATION Scharfetter-Gummel Method}

\author{
Jinn-Liang Liu \\ Institute for Computational and Modeling Science, National Tsing Hua \\ University, Hsinchu 300, Taiwan.E-mail: jlliu@mx.nthu.edu.tw
}

11/14/2008, 1/5/2010, 9/3/2011, 12/6/2016

\begin{abstract}
The Scharfetter-Gummel method provides an optimum way to discretize the driftdiffusion (or Nernst-Planck) equation for charged particle transport in semiconductor devices (or ionic flow in biological ion channels). This is an exponential fitting method usually called in the literature of convection-dominated fluid models.
\end{abstract}

The steady state 1D Nernst-Planck (drift-diffusion) equation of cations (or holes) in an ion channel (or a semiconductor device) is

$$
-\frac{d}{d x} J(x)=0, \quad \forall x \in(0, l)
$$

where

$$
J(x)=-D \frac{d C(x)}{d x}+\mu E C(x)
$$

is the flux density of cations, $C(x)$ is an unknown concentration (distribution) function of cations, $E=-\frac{d \phi(x)}{d x}$ is the electric field, $\phi(x)$ is the electrostatic potential, $\mu$ is the hole mobility, and $D$ is the diffusion coefficient of cations. The Einstein relation of charged particles is $D=\mu k_{B} T / q$, where $k_{B}$ is the Boltzmann constant, $T$ is absolute temperature, and $q$ is the charge on each particle (cation or anion).

Assuming that $\mu, E, D, J$ are constant within the interval $\left[x_{i}, x_{i+1}\right] \subset[0, l]$, we have from (2)

$$
\frac{d C(x)}{d x}=\frac{\mu E}{D} C(x)-\frac{J}{D}=b C(x)-\frac{J}{D}
$$

which implies that 


$$
\begin{aligned}
\frac{1}{C(x)-\frac{J}{b D}} \frac{d C(x)}{d x} & =b, \quad b=\frac{\mu E}{D} \\
\frac{d}{d x} \ln \left|C(x)-\frac{J}{b D}\right| & =b \\
\ln \left|C(x)-\frac{J}{b D}\right| & =b x+c, \quad c \text { is a constant, } \\
C(x)-\frac{J}{b D} & = \pm e^{b x+c} \text { on }\left[x_{i}, x_{i+1}\right] .
\end{aligned}
$$

Therefore, the flux $J$ at the grid point $x_{i+\frac{1}{2}}=\frac{x_{i}+x_{i+1}}{2}\left(\right.$ denoted by $\left.J_{i+\frac{1}{2}}\right)$ can be written as

$$
\begin{aligned}
\frac{C_{i+1}-\frac{J_{i+\frac{1}{2}}}{b D}}{C_{i}-\frac{J_{i+\frac{1}{2}}}{b D}} & =e^{b h_{i}}, \quad h_{i}=x_{i+1}-x_{i}, C_{i}=C\left(x_{i}\right), \\
C_{i+1}-\frac{J_{i+\frac{1}{2}}}{b D} & =e^{b h_{i}}\left(C_{i}-\frac{J_{i+\frac{1}{2}}}{b D}\right) \\
\left(e^{b h_{i}}-1\right) \frac{J_{i+\frac{1}{2}}}{b D} & =\left(-C_{i+1}+e_{i}^{b h_{i}} C_{i}\right) \\
J_{i+\frac{1}{2}} & =\frac{b D}{\left(e^{b h_{i}}-1\right)}\left(-C_{i+1}+e_{i}^{b h_{i}} C_{i}\right) \\
& =\frac{D}{h_{i}}\left[\frac{-b h_{i}}{\left(e^{b h_{i}}-1\right)} C_{i+1}+\frac{-b h_{i}}{\left(e^{-b h_{i}}-1\right)} C_{i}\right] \\
& =\frac{D}{h_{i}}\left[-B\left(-t_{i}\right) C_{i+1}+B\left(t_{i}\right) C_{i}\right]
\end{aligned}
$$

where

$$
\begin{aligned}
b & =\frac{\mu E}{D}=-\beta \frac{d \phi}{d x}=-\beta \frac{\phi_{i+1}-\phi_{i}}{h_{i}}, \beta=\frac{q}{k_{B} T} \\
t_{i} & =\beta \Delta \phi_{i}, \Delta \phi_{i}=\phi_{i+1}-\phi_{i} \\
B(t) & =\frac{t}{e^{t}-1} \text { is the Bernoulli function. }
\end{aligned}
$$

For uniform mesh, i.e., $h_{i-1}=h_{i}$, the Scharfetter-Gummel method for (1) at $x_{i}$ is thus

$$
\begin{aligned}
\frac{d}{d x} J\left(x_{i}\right) & \approx \frac{1}{\frac{h_{i-1}+h_{i}}{2}}\left(J_{i+\frac{1}{2}}-J_{i-\frac{1}{2}}\right)=0 \Rightarrow a_{i-1} C_{i-1}+a_{i} C_{i}+a_{i+1} C_{i+1}=0 \\
J_{i+\frac{1}{2}} & =D\left[-B\left(-t_{i}\right) C_{i+1}+B\left(t_{i}\right) C_{i}\right], \quad J_{i-\frac{1}{2}}=D\left[-B\left(-t_{i-1}\right) C_{i}+B\left(t_{i-1}\right) C_{i-1}\right] \\
a_{i-1} & =-B\left(t_{i-1}\right), \quad a_{i}=B\left(-t_{i-1}\right)+B\left(t_{i}\right), \quad a_{i+1}=-B\left(-t_{i}\right) .
\end{aligned}
$$


Appendix C

MODE PROPAGATION CONSTANT

SLAB WAVEGUIDE ELECTROMAGNETIC APPROACH

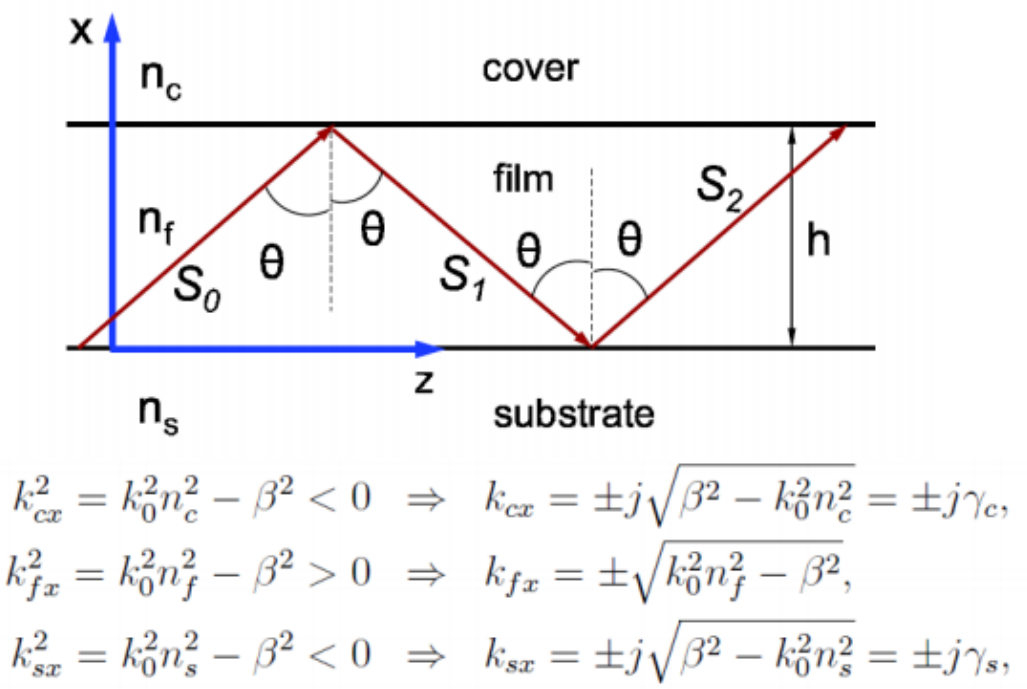

$$
\vec{U}= \begin{cases}\vec{U}_{c} e^{-\gamma_{c}(x-h)} e^{-j \beta z}, & x>h, \\ {\left[\vec{U}_{f 1} e^{-j k_{f x} x}+\vec{U}_{f 2} e^{+j k_{f x} x}\right] e^{-j \beta z},} & 0<x<h, \\ \vec{U}_{s} e^{\gamma_{s} x} e^{-j \beta z}, & x<0,\end{cases}
$$

Prof. Elias N. Glytsis, School of ECE, NTUA

Figure C.1: Wave Solutions in Material

[36] 


\section{SLAB WAVEGUIDE ELECTROMAGNETIC APPROACH} TE Modes

$$
\begin{aligned}
& \text { Dispersion Equation } k_{0} \max \left\{n_{c}, n_{s}\right\}<\beta<k_{0} n_{f} \\
& \underbrace{\left[\begin{array}{cccc}
-1 & e^{-j k_{f x} h} & e^{+j k_{f x} h} & 0 \\
j \gamma_{c} & k_{f x} e^{-j k_{f x} h} & -k_{f x} e^{+j k_{f x} h} & 0 \\
0 & 1 & 1 & -1 \\
0 & k_{f x} & -k_{f x} & -j \gamma_{s}
\end{array}\right]}_{\tilde{\mathcal{A}}_{T E}\left(\beta^{2}\right)}\left[\begin{array}{c}
E_{c} \\
E_{f 1} \\
E_{f 2} \\
E_{s}
\end{array}\right]=\left[\begin{array}{l}
0 \\
0 \\
0 \\
0
\end{array}\right] \\
& \operatorname{det}\left\{\tilde{\mathcal{A}}_{T E}\left(\beta^{2}\right)\right\}=0 \Longrightarrow \tan \left(k_{f x} h\right)=\frac{\frac{\gamma_{s}}{k_{f x}}+\frac{\gamma_{c}}{k_{f x}}}{1-\frac{\gamma_{s}}{k_{f x}} \frac{\gamma_{c}}{k_{f x}}} . \\
& \vec{E}_{\nu}=\hat{y} E_{0} \begin{cases}\cos \left(k_{f x} h-\phi_{f s}\right) e^{-\gamma_{c}(x-h)} e^{-j \beta_{\nu} z}, & x>h, \\
\cos \left(k_{f x} x-\phi_{f s}\right) e^{-j \beta_{\nu} z}, & 0<x<h, \\
\cos \phi_{f s} e^{\gamma_{s} x} e^{-j \beta_{\nu} z}, & x<0,\end{cases}
\end{aligned}
$$

Prof. Elias N. Glytsis, School of ECE, NTUA

Figure C.2: Transcendental Equation from Matrix Determinant [36] 
Appendix D

LASING PLOTS

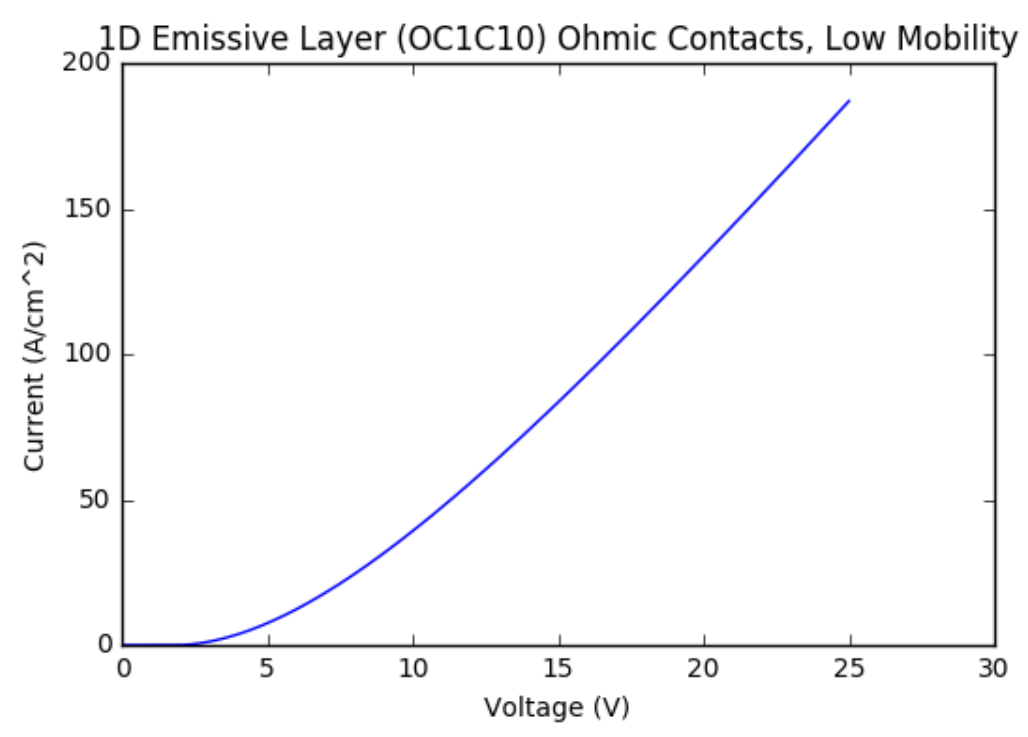

Figure D.1: IV-Plot of 1D Emissive Layer (OC1C10) Ohmic Contacts Low Mobility

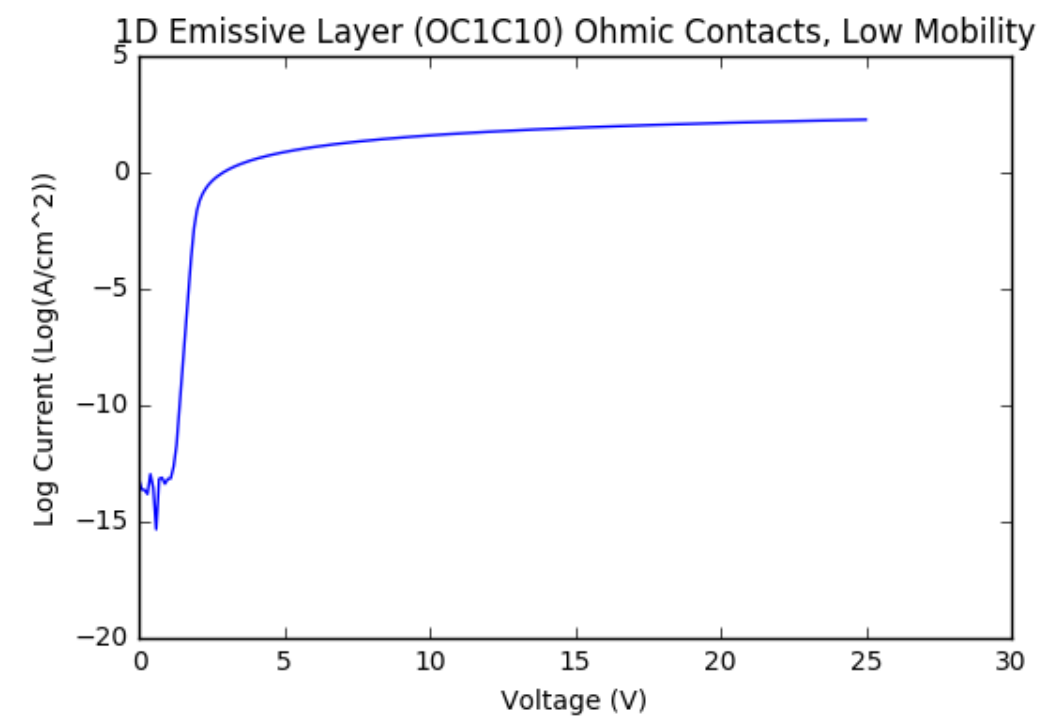

Figure D.2: Log IV-Plot of 1D Emissive Layer (OC1C10) Ohmic Contacts Low Mobility 


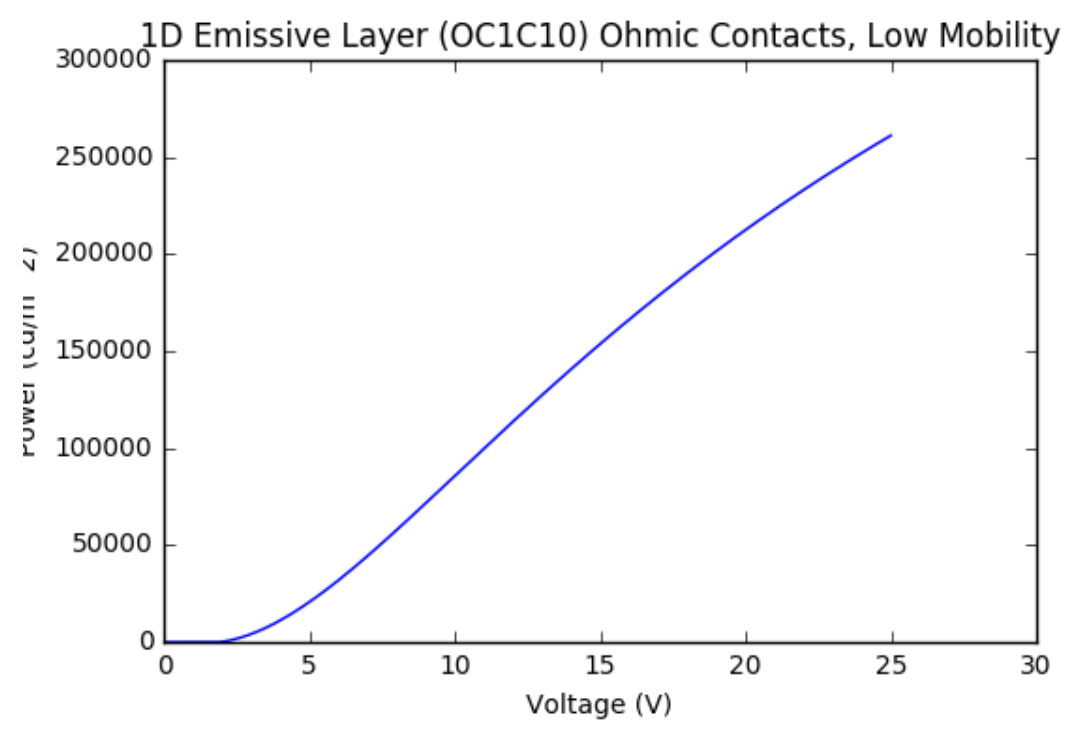

Figure D.3: PV-Plot of 1D Emissive Layer (OC1C10) Ohmic Contacts Low Mobility

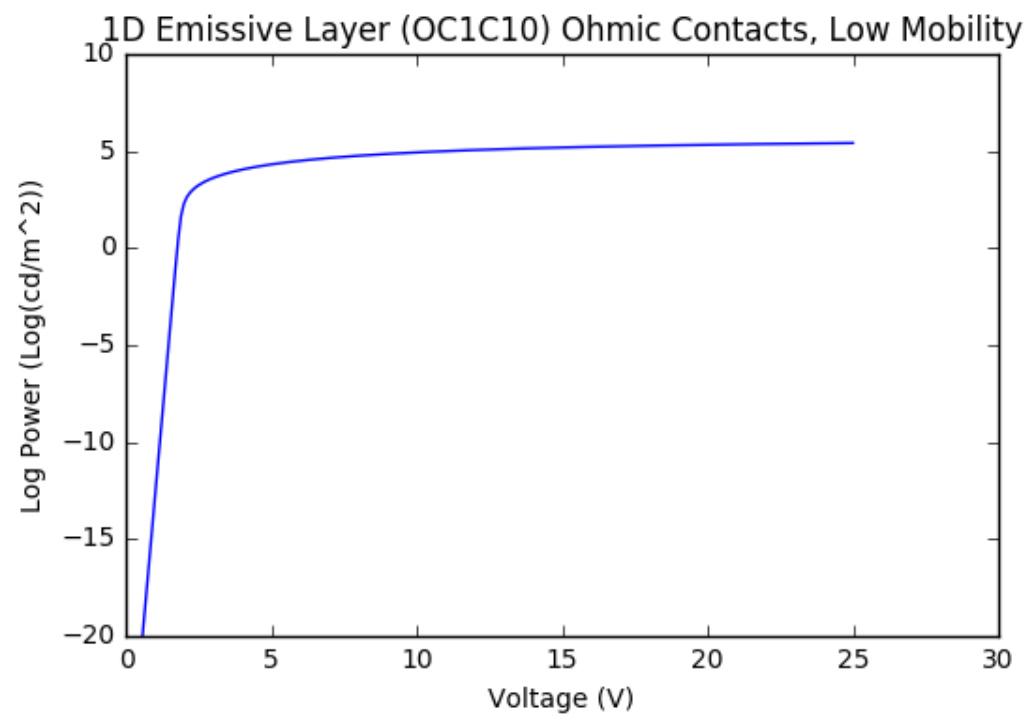

Figure D.4: Log PV-Plot of 1D Emissive Layer (OC1C10) Ohmic Contacts Low Mobility 


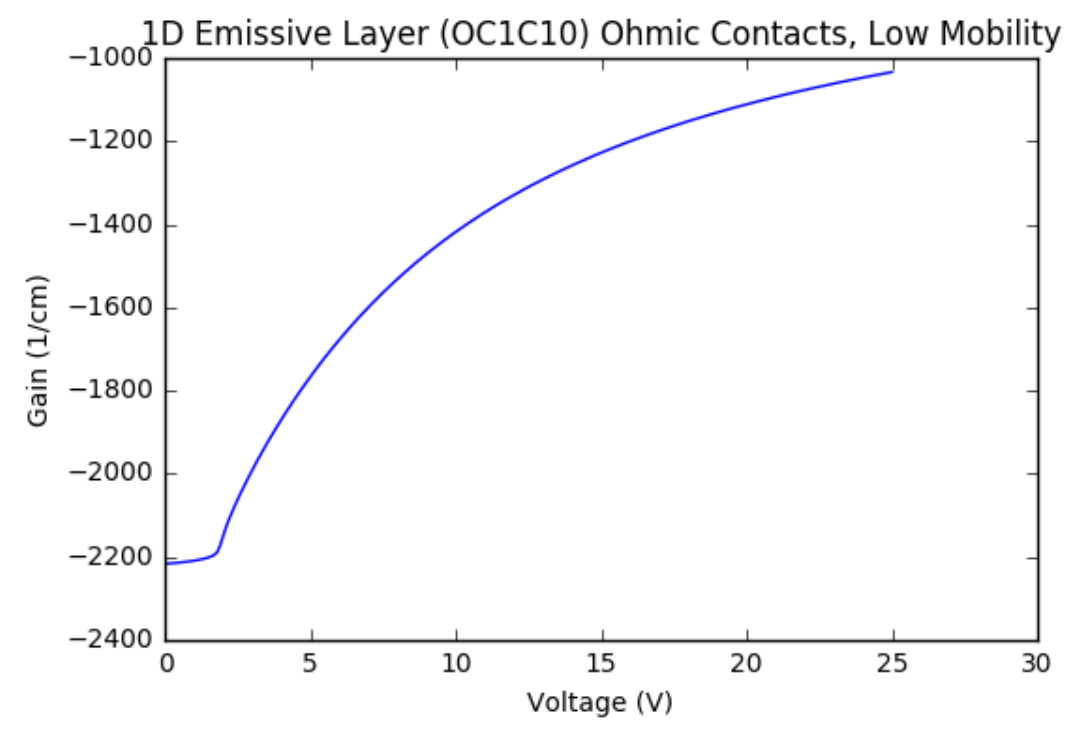

Figure D.5: GV-Plot of 1D Emissive Layer (OC1C10) Ohmic Contacts Low Mobility

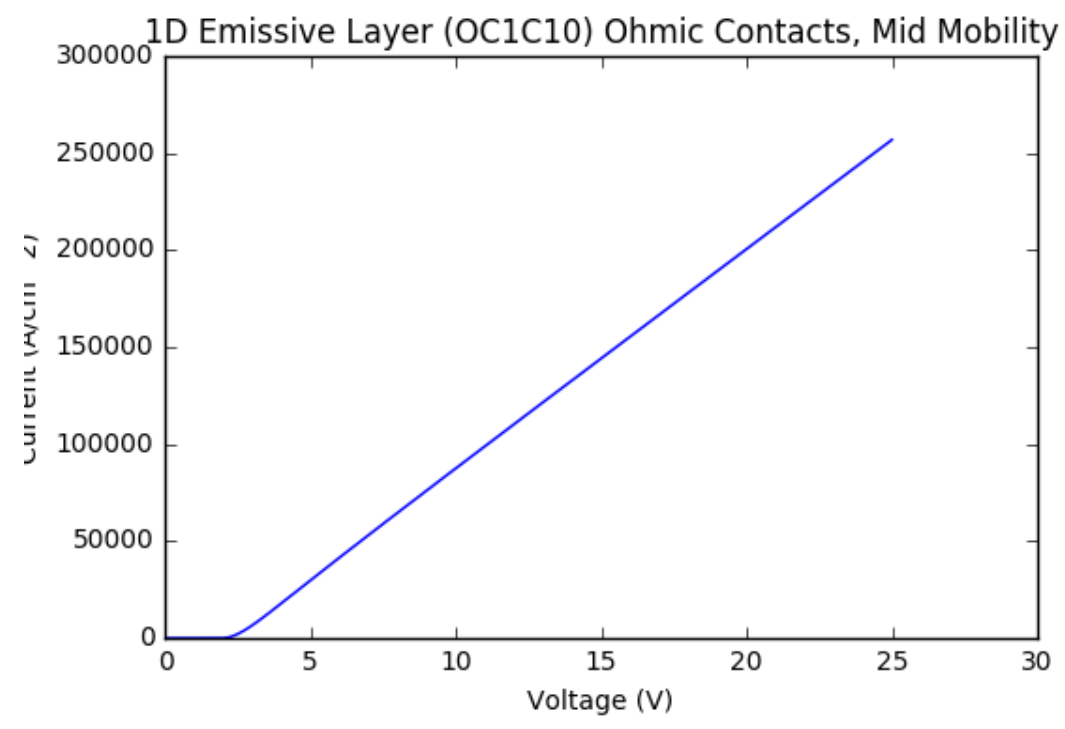

Figure D.6: IV-Plot of 1D Emissive Layer (OC1C10) Ohmic Contacts Mid Mobility 


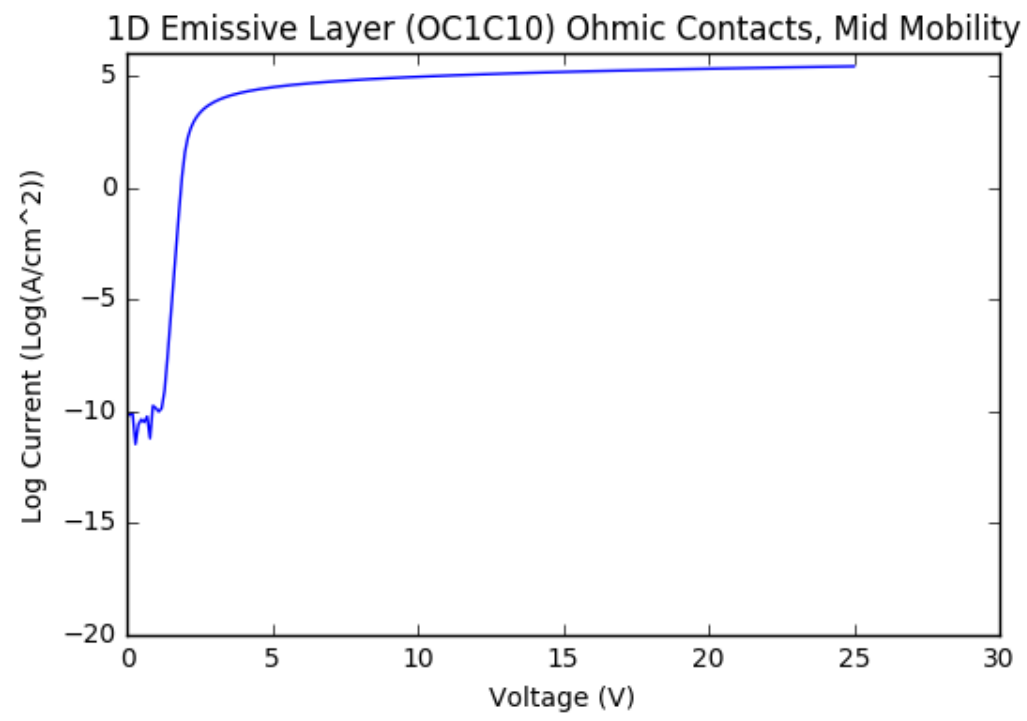

Figure D.7: Log IV-Plot of 1D Emissive Layer (OC1C10) Ohmic Contacts Mid Mobility

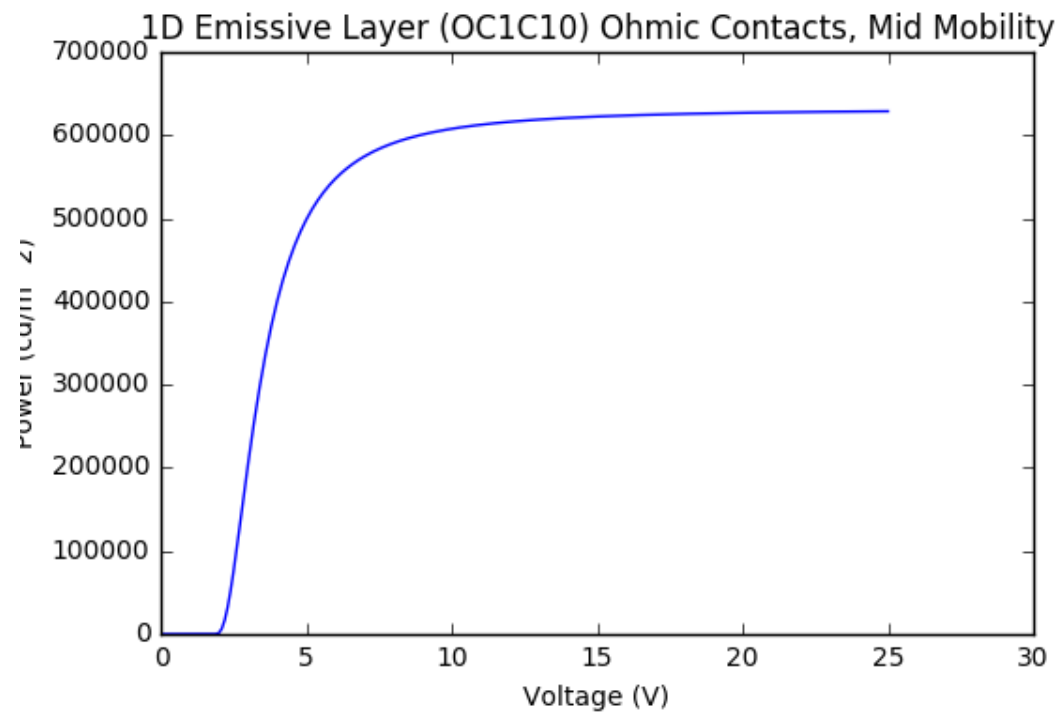

Figure D.8: PV-Plot of 1D Emissive Layer (OC1C10) Ohmic Contacts Mid Mobility 


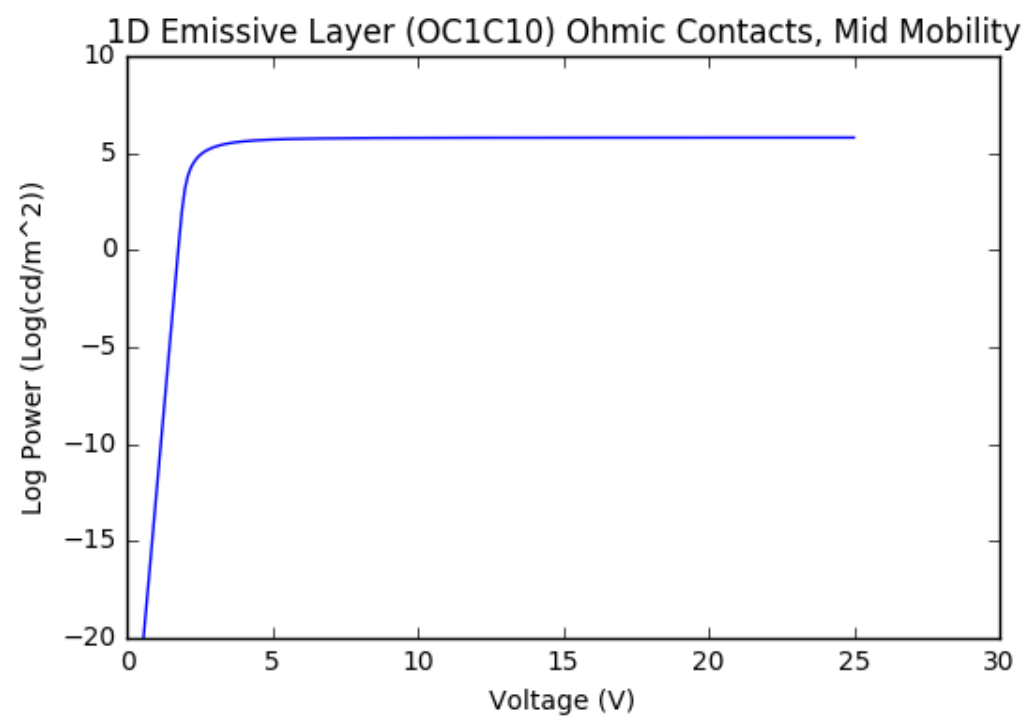

Figure D.9: Log PV-Plot of 1D Emissive Layer (OC1C10) Ohmic Contacts Mid Mobility

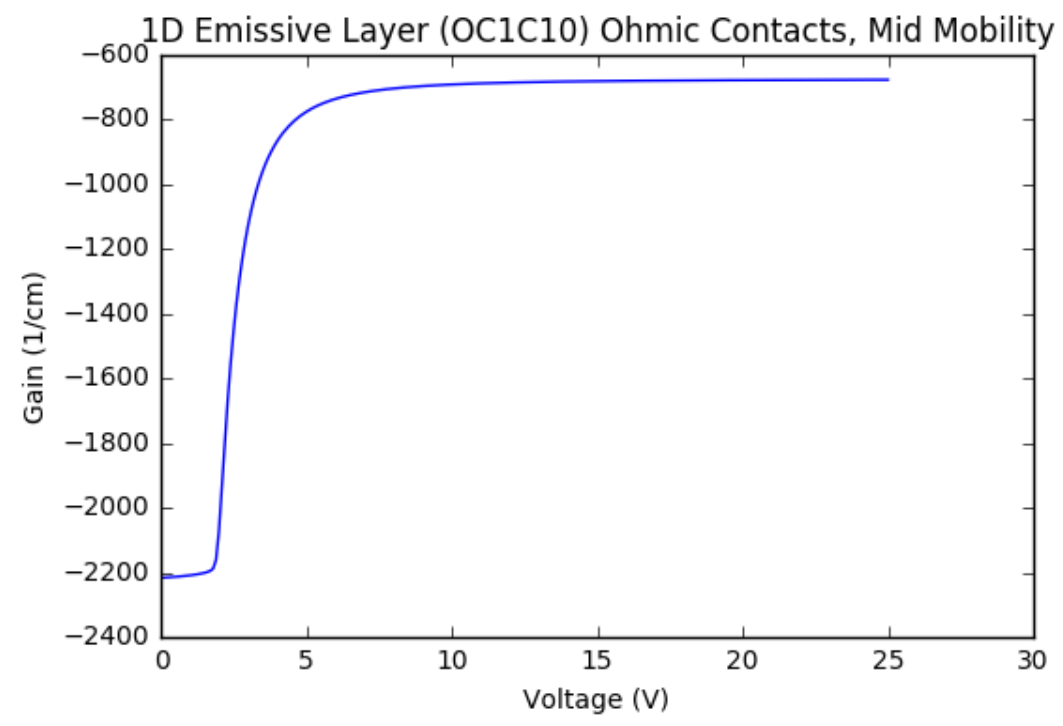

Figure D.10: GV-Plot of 1D Emissive Layer (OC1C10) Ohmic Contacts Mid Mobility 


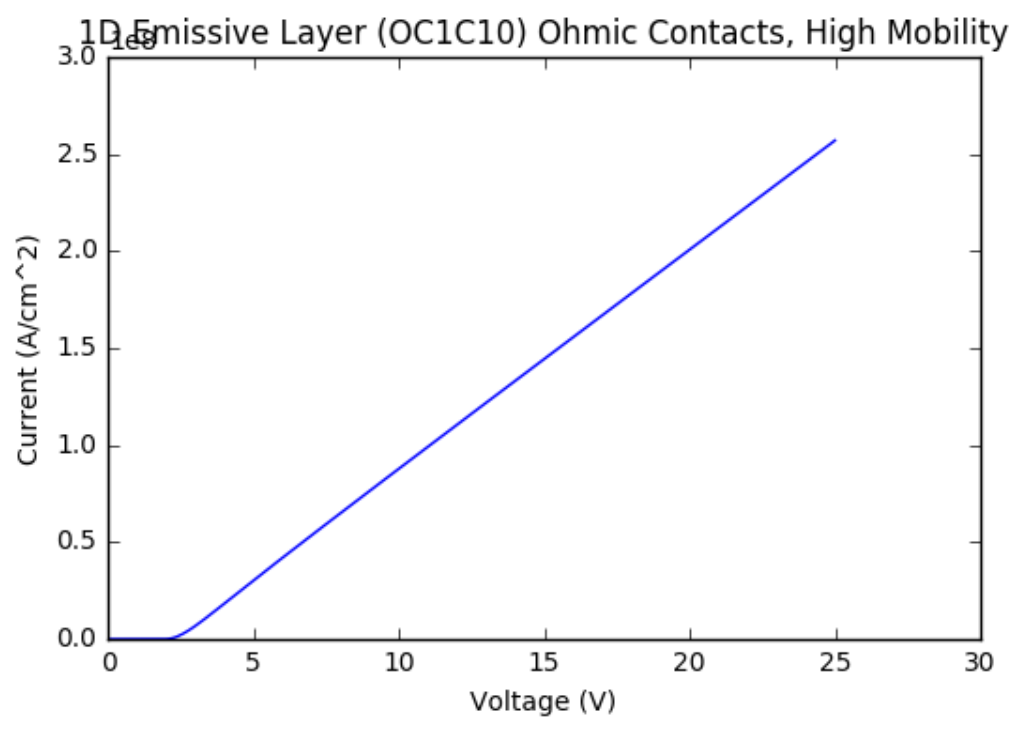

Figure D.11: IV-Plot of 1D Emissive Layer (OC1C10) Ohmic Contacts High Mobility

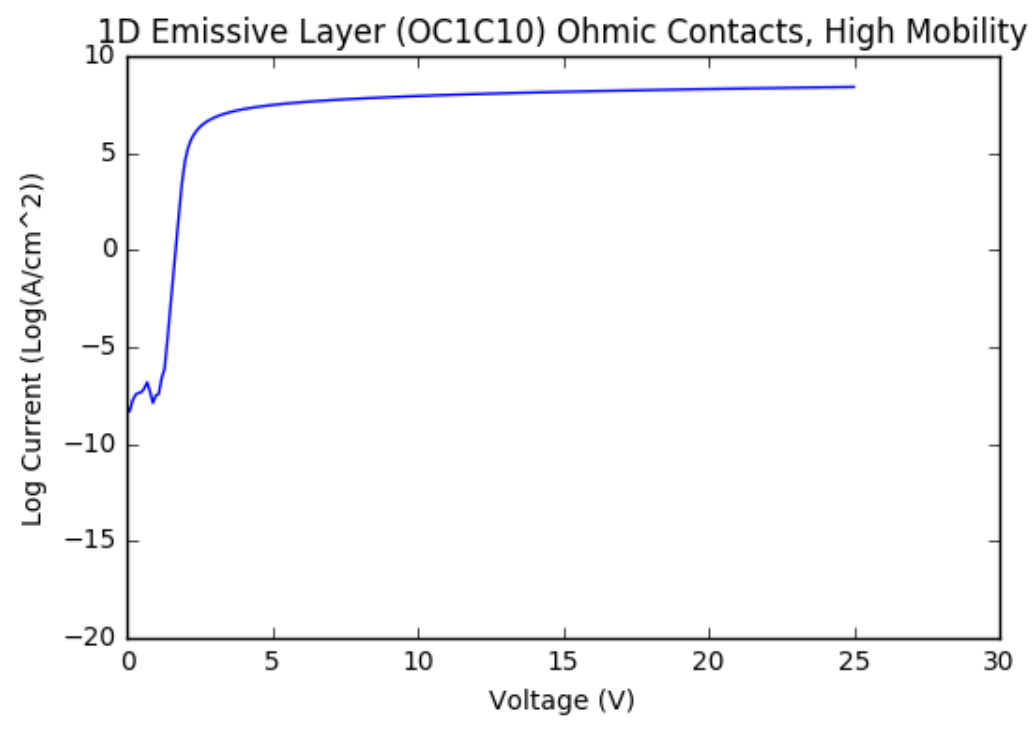

Figure D.12: Log IV-Plot of 1D Emissive Layer (OC1C10) Ohmic Contacts High Mobility 


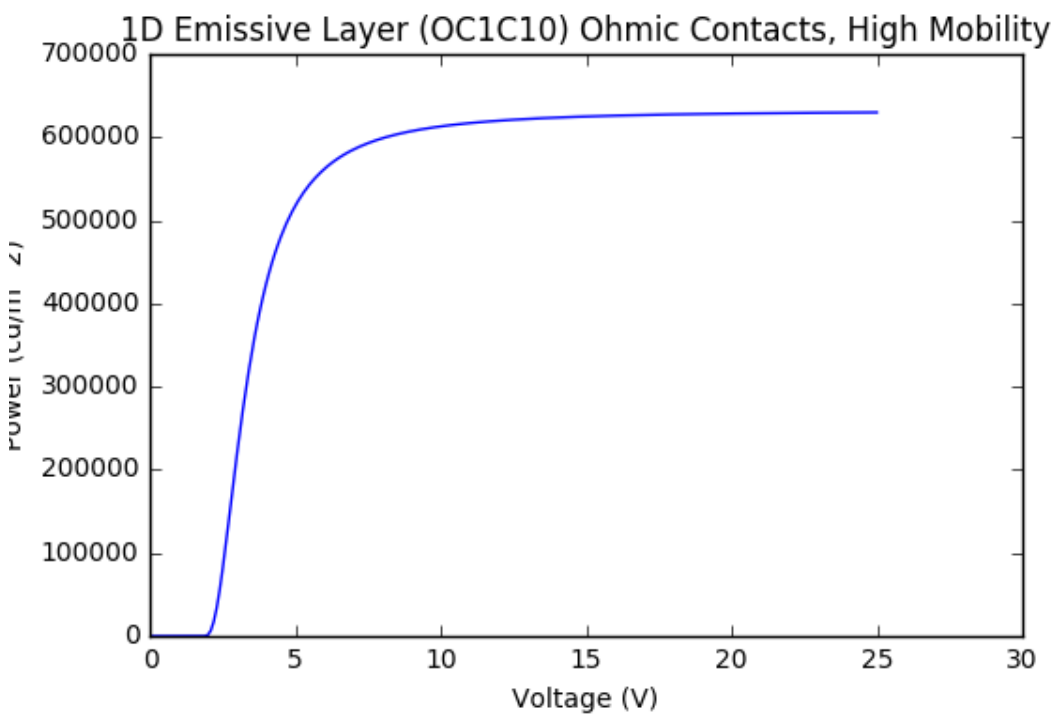

Figure D.13: PV-Plot of 1D Emissive Layer (OC1C10) Ohmic Contacts High Mobility

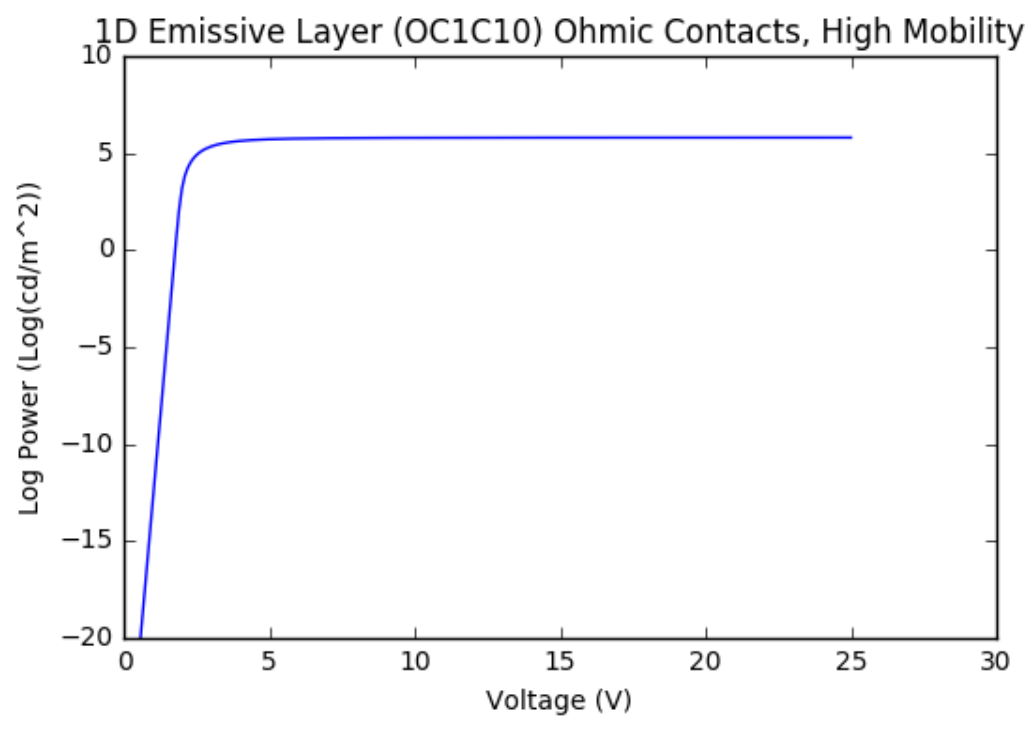

Figure D.14: Log PV-Plot of 1D Emissive Layer (OC1C10) Ohmic Contacts High Mobility 


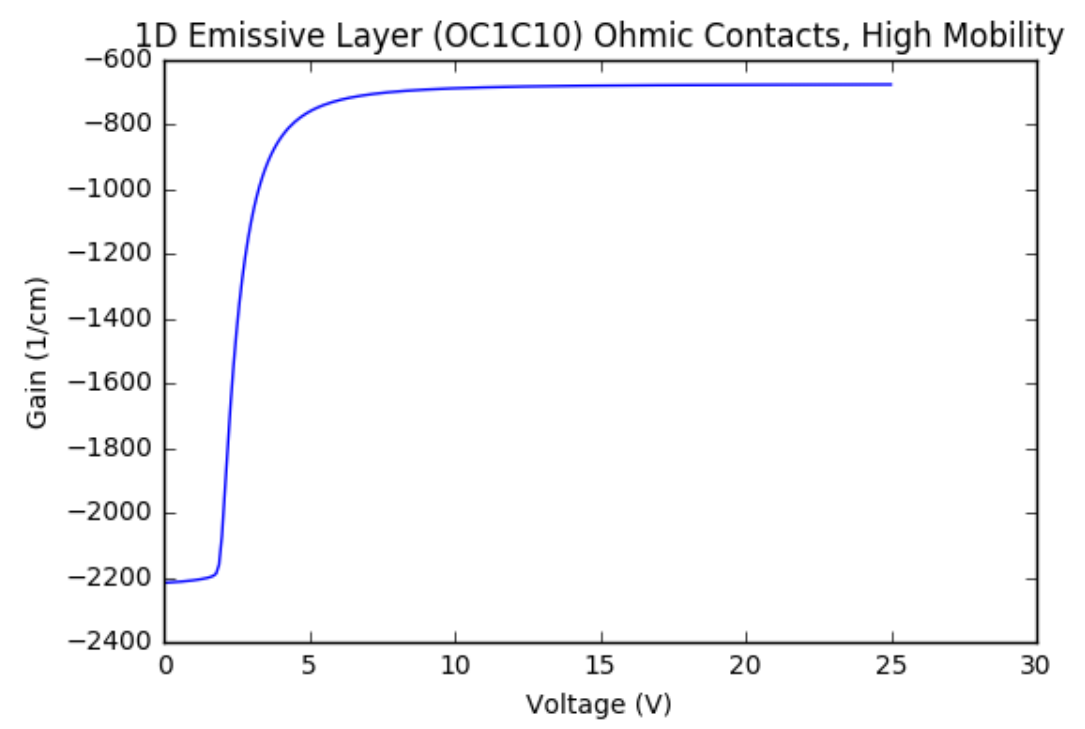

Figure D.15: GV-Plot of 1D Emissive Layer (OC1C10) Ohmic Contacts High Mobility

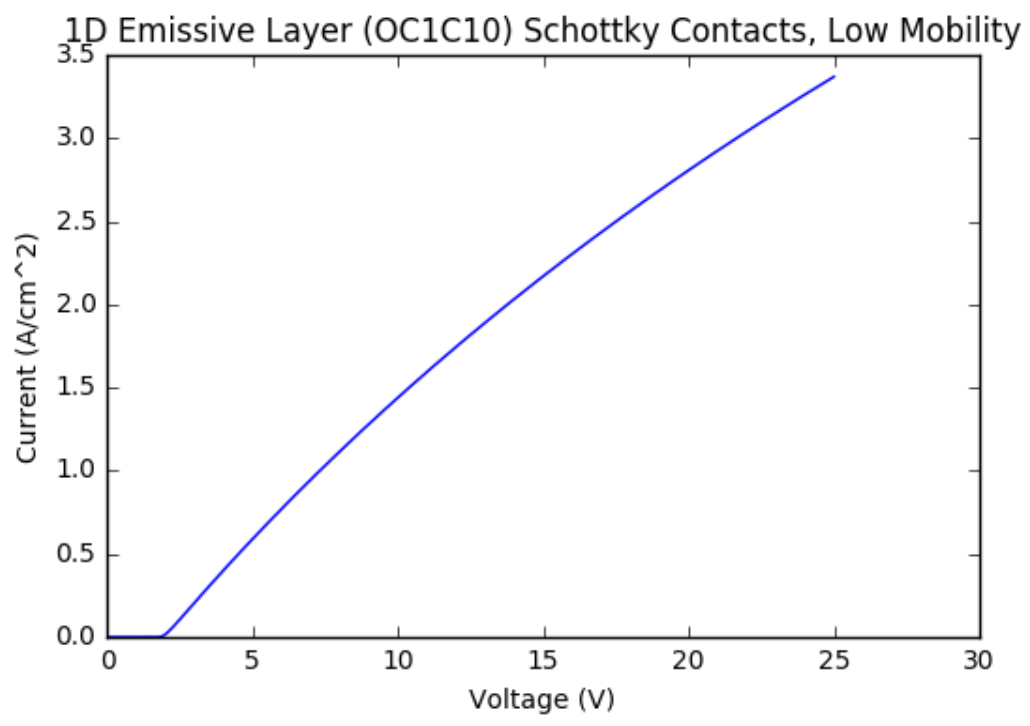

Figure D.16: IV-Plot of 1D Emissive Layer (OC1C10) Schottky Contacts Low Mobility 


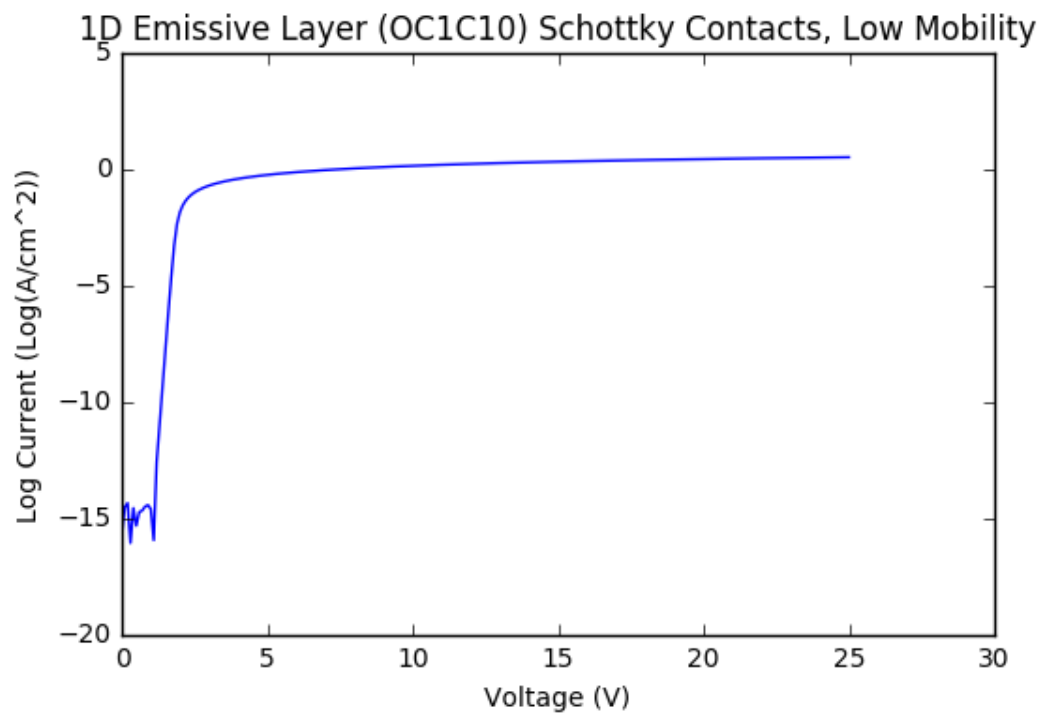

Figure D.17: Log IV-Plot of 1D Emissive Layer (OC1C10) Schottky Contacts Low Mobility

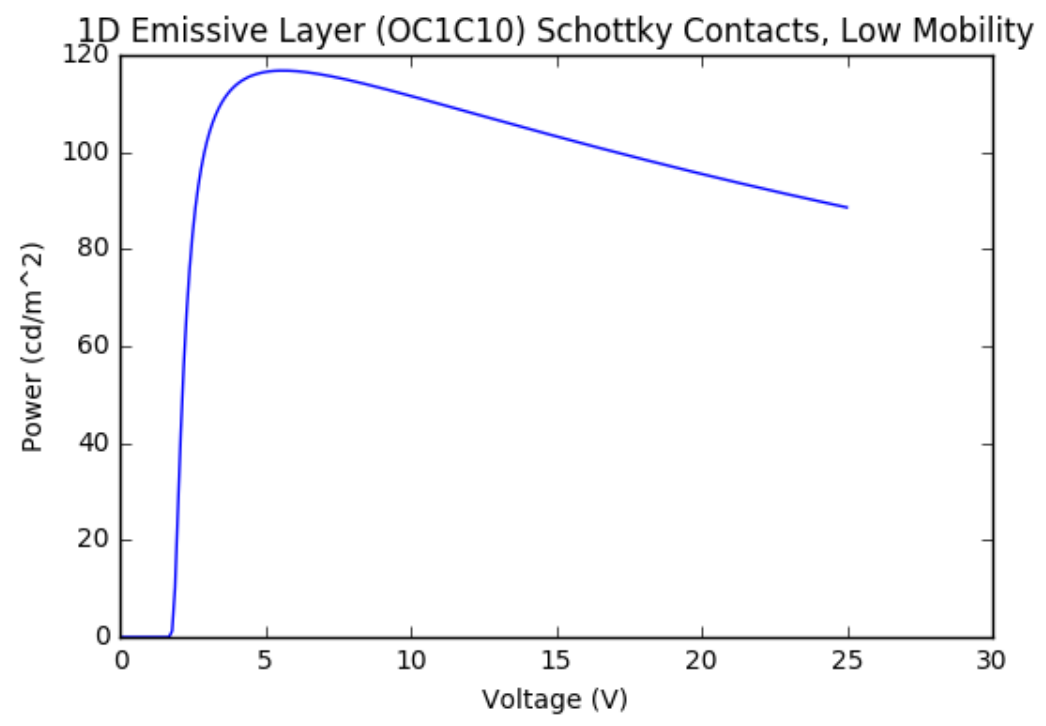

Figure D.18: PV-Plot of 1D Emissive Layer (OC1C10) Schottky Contacts Low Mobility 


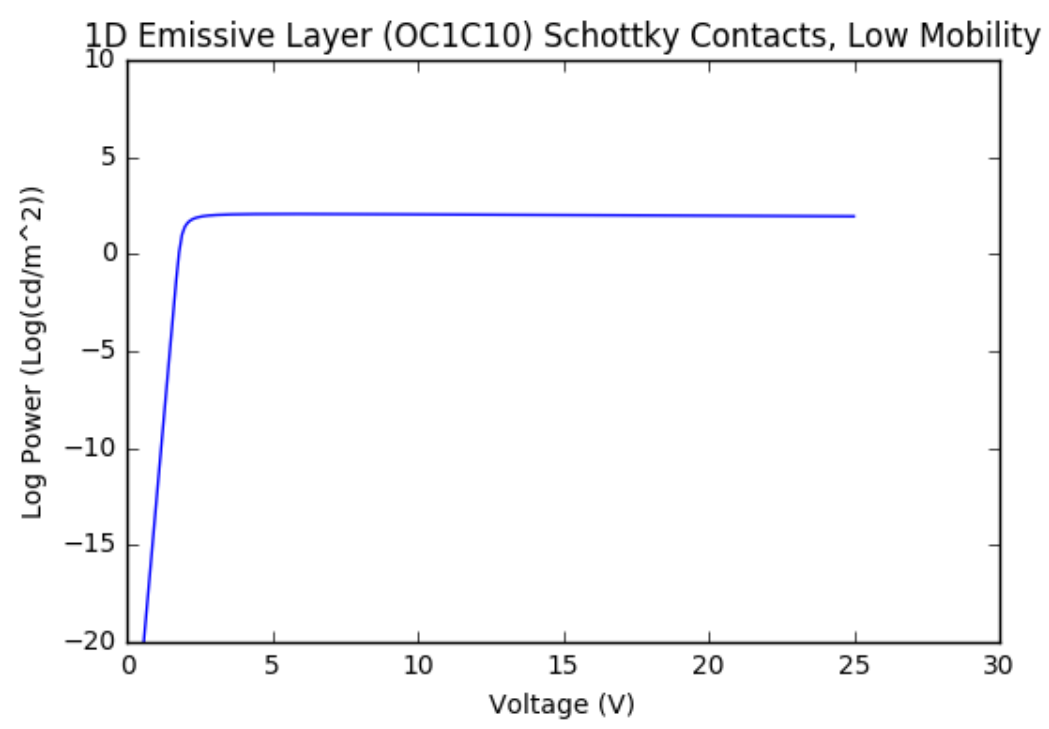

Figure D.19: Log PV-Plot of 1D Emissive Layer (OC1C10) Schottky Contacts Low Mobility

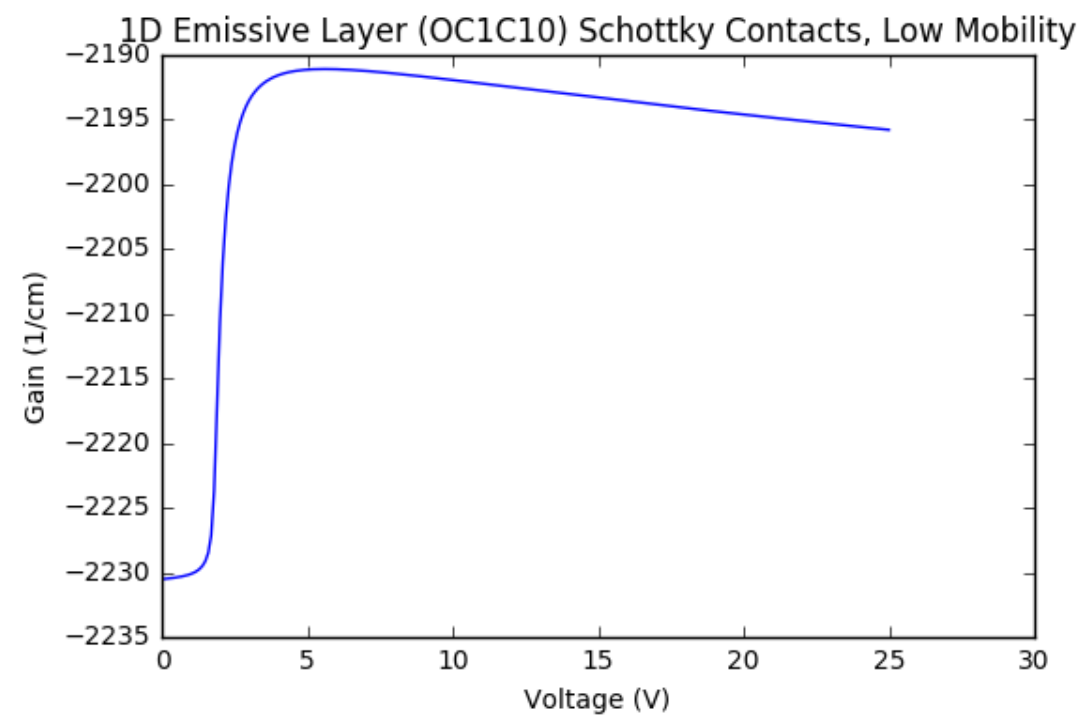

Figure D.20: GV-Plot of 1D Emissive Layer (OC1C10) Schottky Contacts Low Mobility 


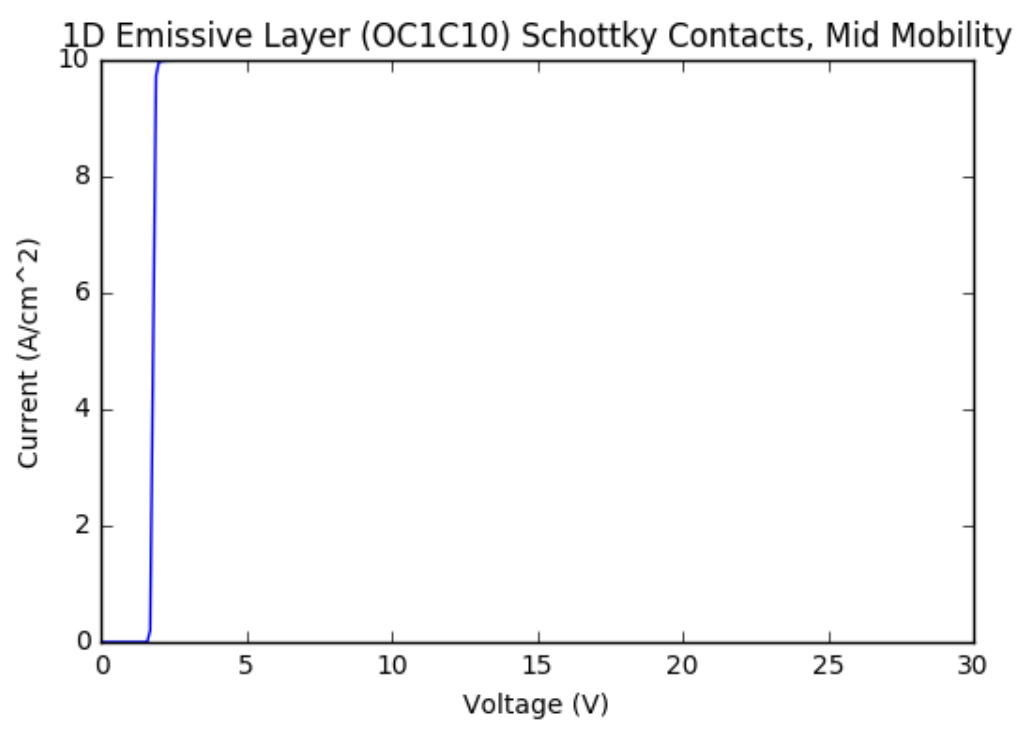

Figure D.21: IV-Plot of 1D Emissive Layer (OC1C10) Schottky Contacts Mid Mobility

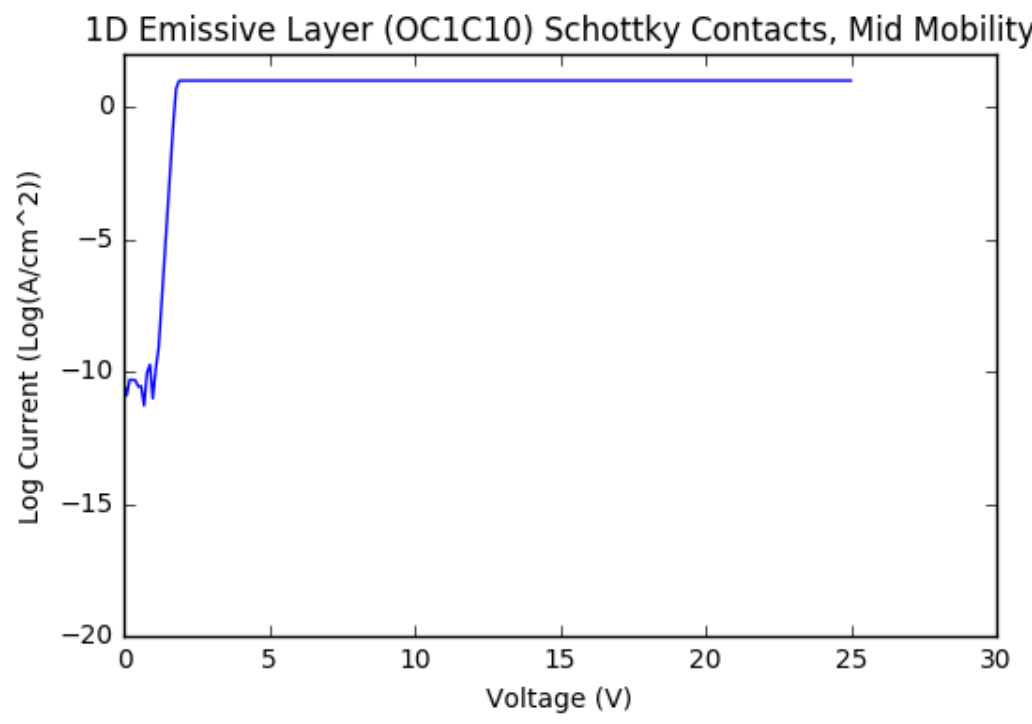

Figure D.22: Log IV-Plot of 1D Emissive Layer (OC1C10) Schottky Contacts Mid Mobility 


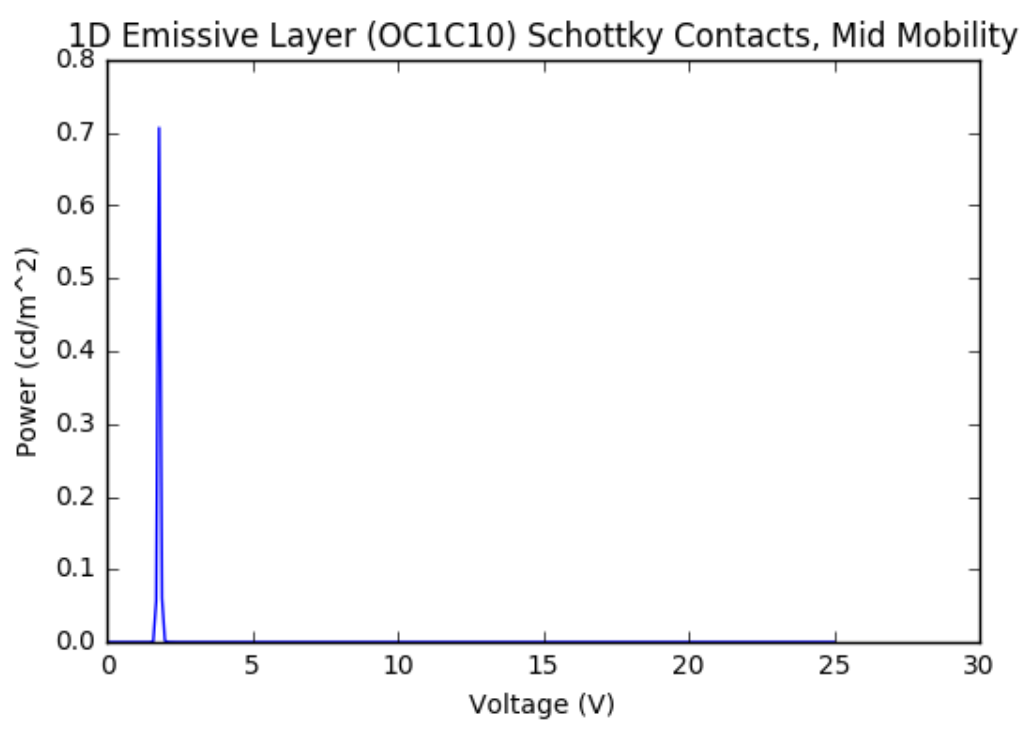

Figure D.23: PV-Plot of 1D Emissive Layer (OC1C10) Schottky Contacts Mid Mobility

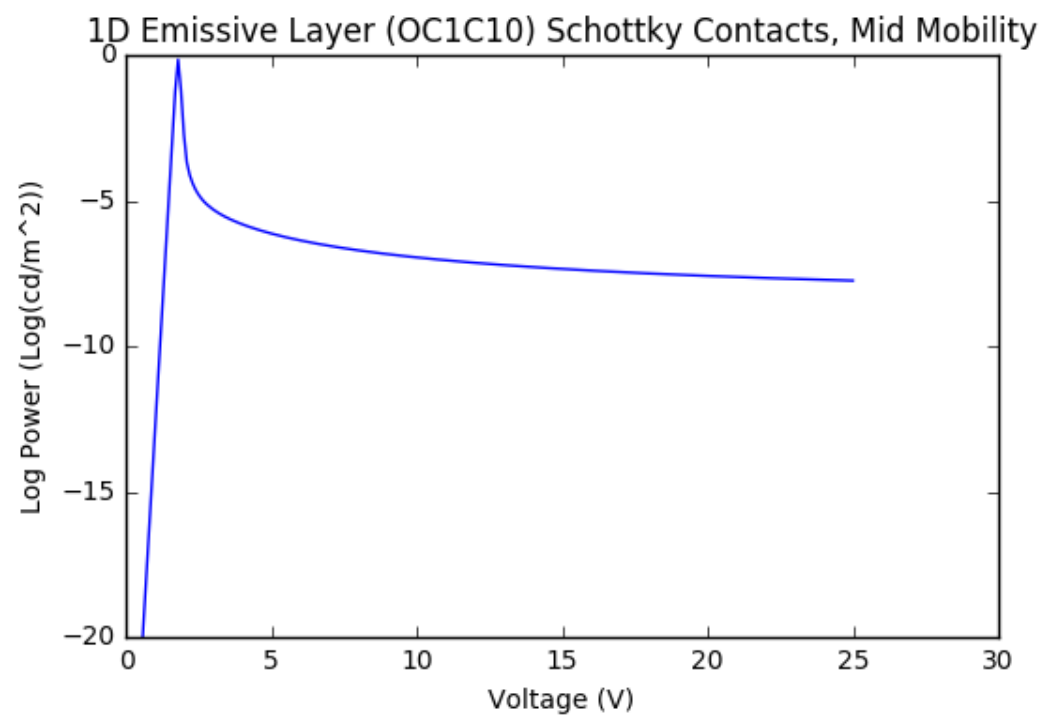

Figure D.24: Log PV-Plot of 1D Emissive Layer (OC1C10) Schottky Contacts Mid Mobility 


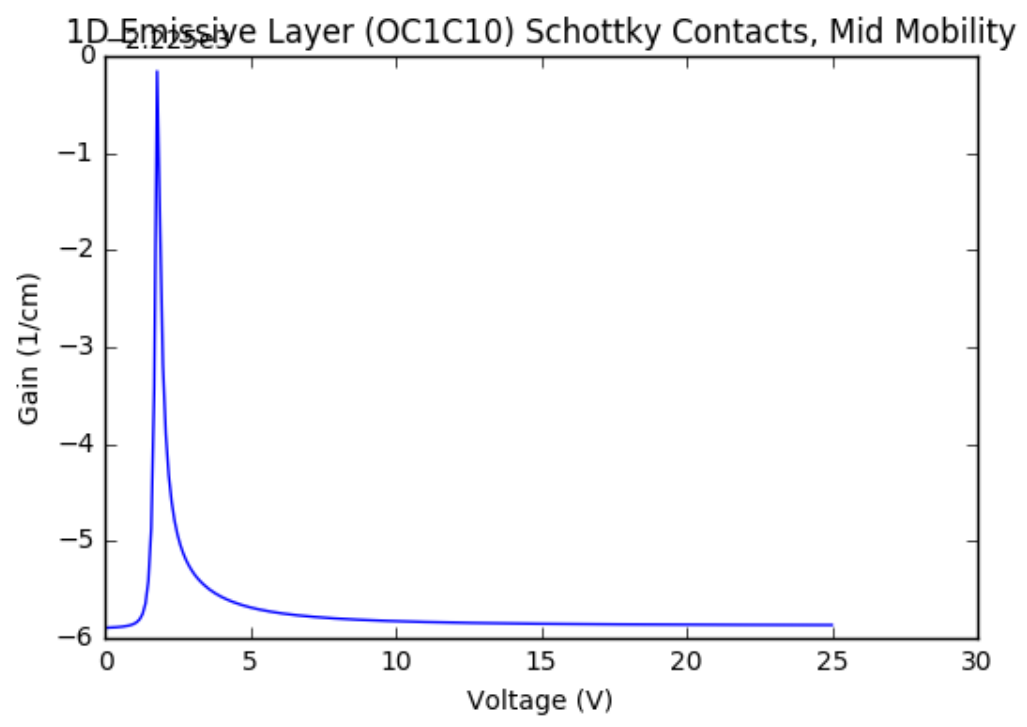

Figure D.25: GV-Plot of 1D Emissive Layer (OC1C10) Schottky Contacts Mid Mobility

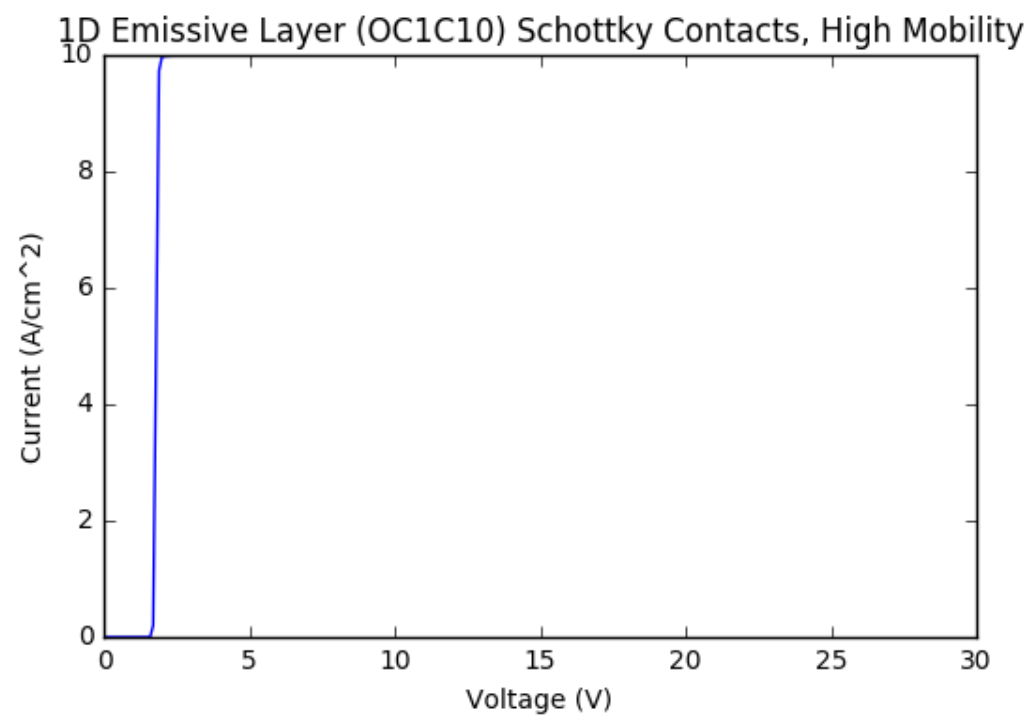

Figure D.26: IV-Plot of 1D Emissive Layer (OC1C10) Schottky Contacts High Mobility 


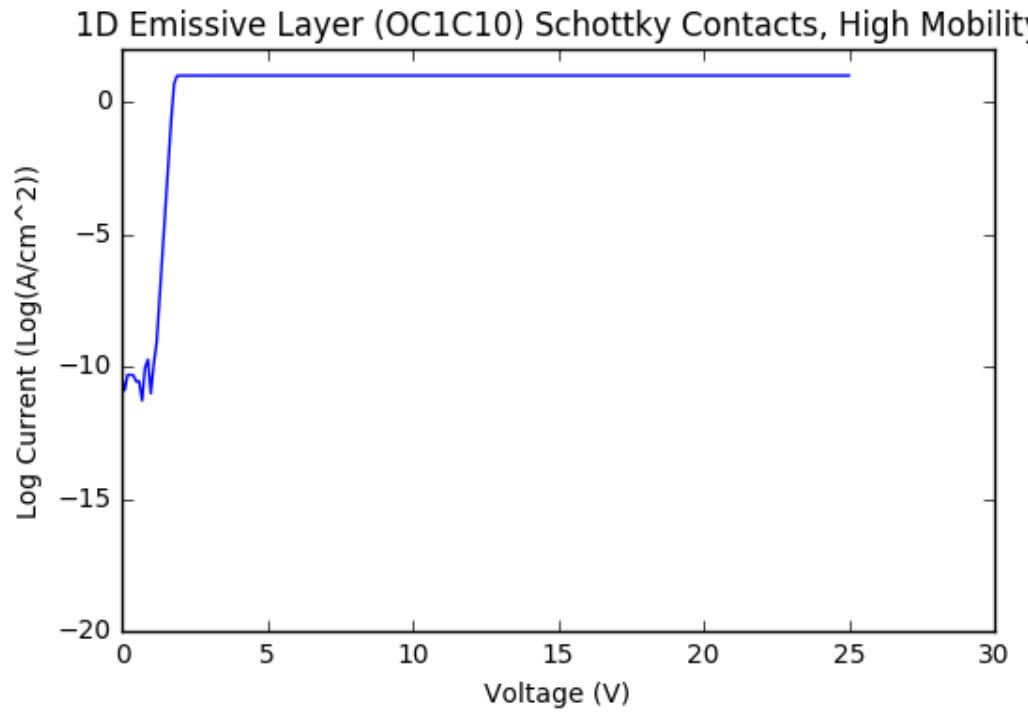

Figure D.27: Log IV-Plot of 1D Emissive Layer (OC1C10) Schottky Contacts High Mobility

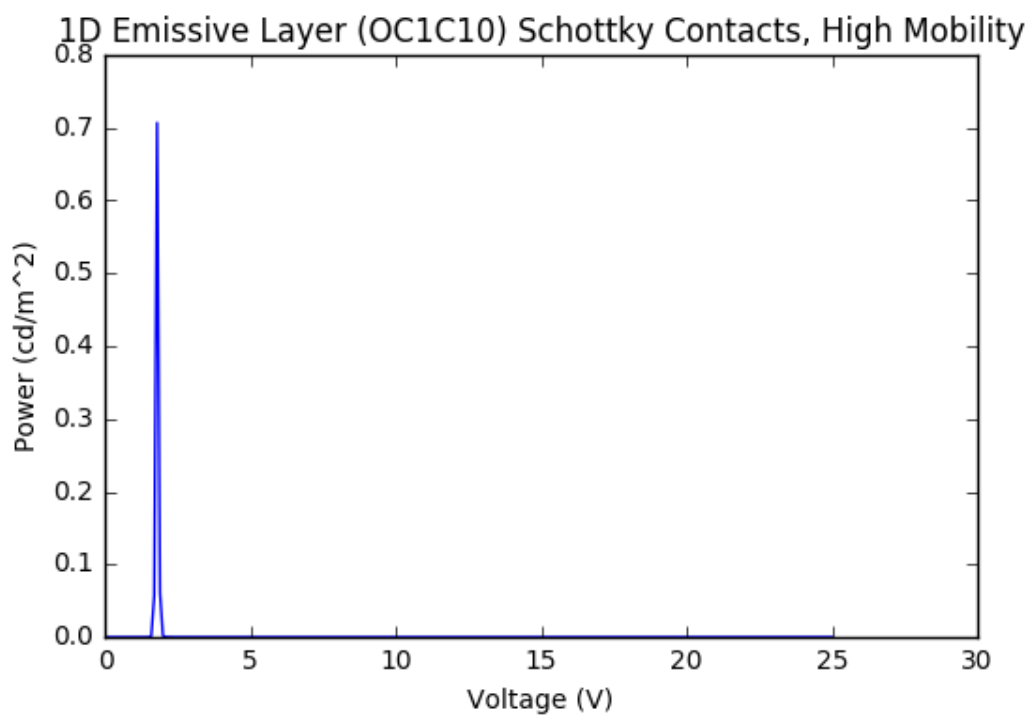

Figure D.28: PV-Plot of 1D Emissive Layer (OC1C10) Schottky Contacts High Mobility 


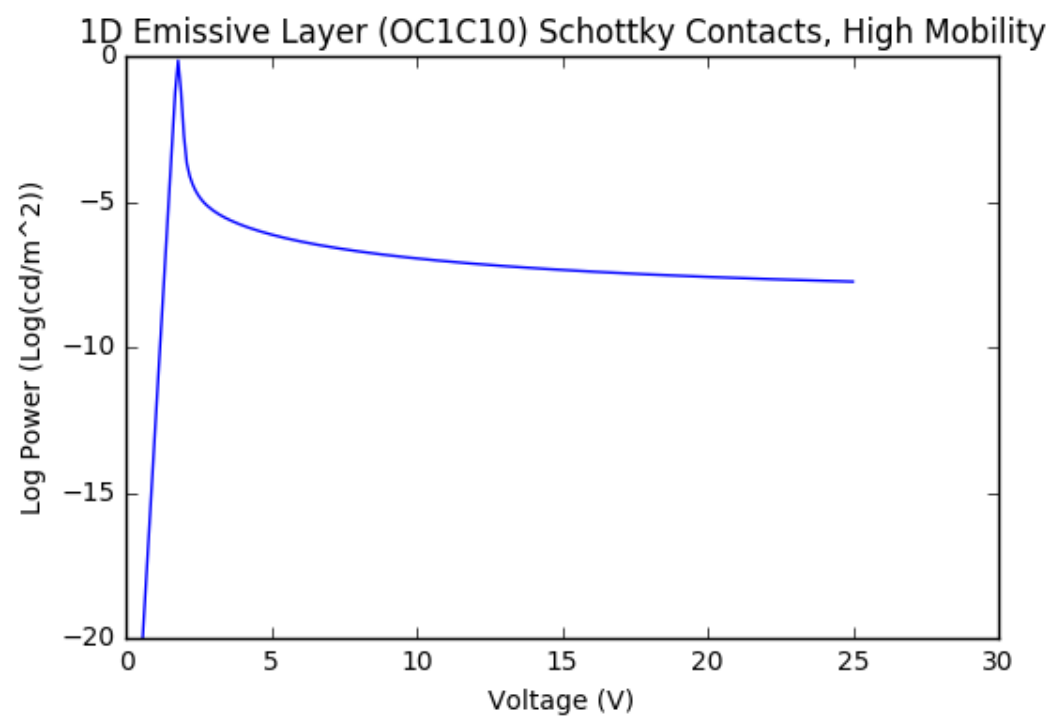

Figure D.29: Log PV-Plot of 1D Emissive Layer (OC1C10) Schottky Contacts High Mobility

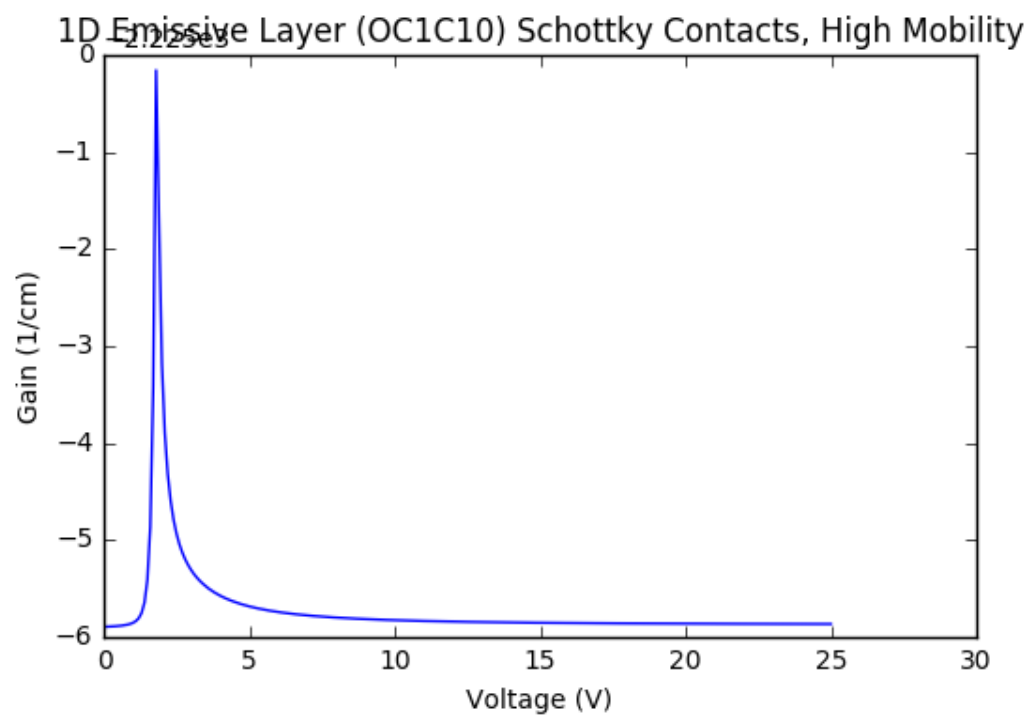

Figure D.30: GV-Plot of 1D Emissive Layer (OC1C10) Schottky Contacts High Mobility 